NISTIR 7288

\title{
Recommended Objective Test Procedures for Road Departure Crash Warning Systems
}

\author{
Sandor Szabo \\ Richard Norcross \\ U.S DEPARTMENT OF COMMERCE \\ Technology Administration \\ National Institute of Standards and Technology \\ Intelligent Systems Division \\ Gaithersburg, MD 20899-8230
}

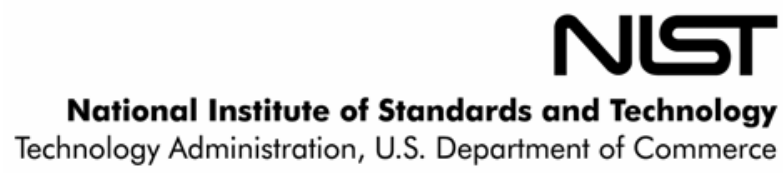




\title{
Recommended Objective Test Procedures for Road Departure Crash Warning Systems
}

\author{
Sandor Szabo \\ Richard Norcross \\ U.S DEPARTMENT OF COMMERCE \\ Technology Administration \\ National Institute of Standards and Technology \\ Intelligent Systems Division \\ Gaithersburg, MD 20899-8230
}

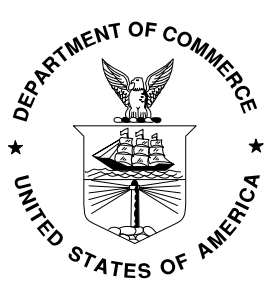

U.S. DEPARTMENT OF COMMERCE Carlos M. Gutierrez, Secretary NATIONAL INSTITUTE OF STANDARDS AND TECHNOLOGY William Jeffrey, Director 


\section{Recommended Objective Test Procedures for Road Departure Crash Warning Systems}

February 3, 2006

\section{Prepared By:}

Sandor Szabo and Richard Norcross

National Institute of Standards and Technology

100 Bureau Drive, Mail Stop 8230

Gaithersburg, MD 20899-8230

This document was prepared for the U.S. Department of Transportation under interagency agreement DTFH61-00-Y-30132. Send comments and/or questions to sandor.szabo@nist.gov.

Certain commercial entities, equipment, or materials may be identified in this document in order to describe an experimental procedure or concept adequately. Such identification is not intended to imply recommendation or endorsement by the National Institute of Standards and Technology, nor is it intended to imply that the entities, materials, or equipment are necessarily the best available for the purpose. 
This page is blank and may be removed for single-sided print out. 


\section{$\underline{\text { History }}$}

June 26, 2002 - First draft of procedures for lateral drift warning system submitted to NHTSA.

September 13, 2002 - Updated previous draft based on information obtained from the contractor's second quarterly review (July 24, 2002). Procedures added for curve speed warning system.

December 18, 2002 - Updated based upon comments from Dave Smith and Lloyd Emery of NHSTA during meetings on December 10, 12 and 18, 2002.

January 31, 2003 - This draft represents latest detailed design of tests at time of February 5 FOT Quarterly Review.

February 18, 2003 - This draft represents latest detailed design at time of trip to visit RDCWS contractor on February 18. The version includes curve speed warning test and the on-road nuisance tests.

November 15, 2005 - This draft represents results from validation tests conducted by the RDCWS contractors and NIST. The on-road tests now exist separately in a Volpe/NIST document. This draft submitted to NHTSA as part of NIST's RDCWS final report 
This page is blank and may be removed for single-sided print out. 
1 Introduction....................................................................................................... 1

2 Definitions................................................................................................................. 4

2.1 Road Definitions ................................................................................. 4

$2.2 \quad$ Vehicle Definitions ............................................................................... 9

2.3 Warning System Definitions................................................................... 13

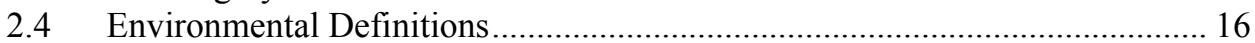

3 Basic Test Organization ....................................................................................... 18

4 Independent Measurement System .................................................................. 20

5 Warning System Evaluations........................................................................ 23

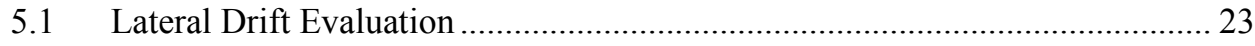

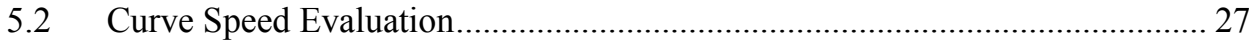

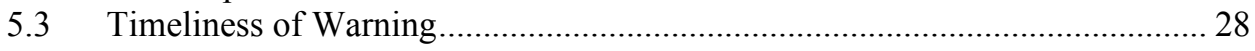

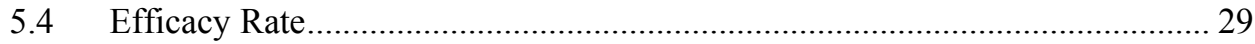

5.5 False Alarm Rate............................................................................... 29

6 Tests for Departure on Roads with Default Minimum AMR............................. 30

6.1 One-Way Straight Road (Default Minimum AMR) ...................................... 31

6.2 Two-Way Straight Road (Default minimum AMR) ...................................... 38

6.3 Curved Road (Default minimum AMR) ............................................... 43

7 Tests for Departures on Roads with Maximum AMR ........................................ 49

7.1 Straight Road with Shoulder (Maximum AMR) ........................................ 50

7.2 Curved Road with Shoulder (Maximum AMR) .................................... 56

8 Tests for Departures Toward an Obstacle ...................................................... 62

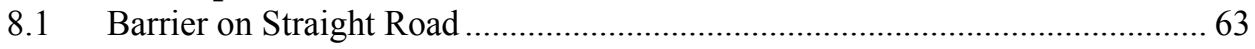

8.2 Barrier on Curved Road .......................................................................... 70

8.3 Obstacle on Shoulder on Straight Road .................................................... 76

8.4 Obstacle on Shoulder on Curved Road ........................................................ 83

9 Tests for Departures on Curves at Excessive Speed............................................ 89

9.1 Excessive Speed on Curved Road.............................................................. 90

9.2 Curve-Branch Detection ......................................................................... 95

References................................................................................................... 99

Appendix A Methods for Determining Road Dimensions and Geometry ............... 101

Appendix B Methods for Determining Lane Marker Characteristics...................... 102 


\section{Introduction}

Under the auspices of the Department of Transportation Intelligent Vehicle Initiative, the National Highway Traffic and Safety Administration (NHTSA) is evaluating the safety benefits of Road Departure Crash Warning Systems (RDCWS). These systems warn inattentive drivers when their vehicle is about to depart the road or collide with a roadside obstacle. To further understand the capabilities and benefits of these warning systems, the NTHSA, with the assistance of the National Institute of Standards and Technology (NIST), is devising test procedures that provide performance data in an objective and quantitative manner. These tests will evolve to reflect new insight into performance measurement and to maintain pace with the capabilities of the warning systems. In the long term, these tests may one-day become part of a standard procedure developers follow to achieve a safety rating similar to the " 5 -star" ratings used in the automotive industry today.

The RDCWS Field Operation Test (FOT) program [1] validated many of the tests included in this report. The developers of the FOT RDCWS used an early version of these procedures to validate system performance. The developers adapted the procedures to meet the time, funding and available track geometry of the FOT. The RDCWS FOT contractor and NIST conducted the tests (called validation tests) at the Transportation Research Center (Maryville Ohio) from October 1, 2003 through April 28, 2004. The successful performance of the system during the tests allowed the FOT to proceed to Phase 2.

These tests utilize considerable research from the international community, NHTSA, the Volpe National Transportation Systems Center, and NIST.

1. An outline of the NIST RDCWS research results appears in a final report titled "Run-Off-Road Collision Avoidance Using IVHS Countermeasures" [2] (referred to as the ROR report in this document)

2. The Road Departure Crash Warning System FOT Request for Applicants issued by the NHTSA [1] contains the performance capabilities expected of a warning system

3. A NHTSA and Volpe National Transportation Systems Center report on an analysis of crash data identifies high-risk crash scenarios [3].

4. Reference [4] is the International Standards Organization test procedure for lane departure warning systems.

A RDCWS consists of the sensor, the alert logic (correctness and timing) and the driver interface. The tests in this document address sensor and alert logic performance only. A complete evaluation of the driver interface would consider how a driver responds to the warning. These tests only evaluate the driver interface from the standpoint of whether the interface presented the warning or not. 
The methods for evaluation of the warning system focus on two areas: correctness and timing. The correctness of each warning issued by the system is either true positive, false positive, false negative or true negative (Table 1). Statistical evaluations, such as efficacy rate (the percentage of warnings that produce the desired effects) and false alarm rate, summarize the results of the ratings.

\begin{tabular}{|c|c|c|}
\hline System Response & $\begin{array}{c}\text { Situations Requiring a } \\
\text { Warning }\end{array}$ & $\begin{array}{c}\text { Situations in Which a } \\
\text { Warning is not Required }\end{array}$ \\
\hline Warning & $\begin{array}{c}\text { True Positive (TP) } \\
\text { Correctly warns of road } \\
\text { departure }\end{array}$ & $\begin{array}{c}\text { False Positive (FP) } \\
\text { False alarm }\end{array}$ \\
\hline No Warning & $\begin{array}{c}\text { False Negative (FN) } \\
\text { Misses road departure }\end{array}$ & $\begin{array}{c}\text { True Negative (TN) } \\
\text { Correctly does not warn of } \\
\text { road departure. }\end{array}$ \\
\hline
\end{tabular}

Table 1 Rating of warning system signals.

The second area of evaluation examines the timing. A warning is of little benefit if it does not provide the driver with sufficient time to avoid the departure. Equations of motion are the basis of the performance metrics and determine the time afforded to a driver. Time limits determine whether a warning is useful.

The methods quantify performance but do not specify the passing grade (e.g., the system must pass $90 \%$ of the tests). The organization interested in the test results should select the pass/fail criteria (some examples are provided in section 5).

Evaluation of the metrics requires real-time measurements, preferably from an independent measurement system (IMS). Using the warning system sensors and processing algorithms may bias the system evaluation. For illustrative purposes, the test procedures include instructions for taking measurements using an IMS developed by NIST.

The ability to detect the road edge is critical to the success of a warning system. For basic warning systems, the road edges are the lane markers in the vehicle's current travel lane and the system issues a warning before the vehicle enters the adjacent lane.

However, driver behavior can produce slight lane excursions and warnings for these instances might become a nuisance. The intent of a road departure crash warning system is to detect true crash conditions, such as driving off-road or drifting into obstacles outside the current lane. The objective to limit nuisance alarms and to focus on real crashes led to the concept of an available maneuver room (AMR), which is the region outside the lane marker that is drivable (e.g., paved shoulder) and clear of obstacles. The AMR added to the outer lane boundary defines the road boundary.

Tests evaluate warning system performance for different types of road-edges. The first set of tests examines the system's ability to detect a road edge defined by the outer lane 
marker under the assumption that there is a minimal amount of AMR. These tests are appropriate for a basic lane departure system.

The next test set examines the system's ability to detect a road edge defined by the transition from pavement to off-road. The size of the shoulder may vary considerably, which would lead to a wide range of warning locations. Previous work [2] suggests a need for consistent warning location. Therefore, the AMR has a default maximum value regardless of the shoulder width.

Another test set examines the system's ability to detect a road edge defined by an obstacle on the shoulder. In one case, the vehicle drifts into an obstacle adjacent to the vehicle, such as a Jersey barrier or guardrail. In the other case, the vehicle drifts into an obstacle up ahead, such as a car parked on the shoulder. In either case, the road edge is an imaginary line at the base of the obstacle and parallel to the lane marker.

The tests above address lateral drift scenarios. The final set of tests evaluates system performance when the vehicle is traveling too fast for an approaching curve, called the curve-speed scenario.

This document has nine sections. The first section is this introduction. Section 2 contains definitions of the terminology used throughout the document. Section 3 contains an overview of the general test structure common to each test. Section 4 describes a measurement system to independently collect data for analyzing warning system performance. Section 5 describes evaluation procedures that are commonly used in lateral drift and curve speed tests. Section 6 contains tests to evaluate departures on roads with no available maneuver room. Section 7 contains tests to evaluate departures on roads with available maneuver room. Section 8 contains tests to evaluate departures towards barriers and obstacles. Section 9 contains tests to evaluate on curves due to excessive speed. 


\section{Definitions}

This section contains definitions of terms used in the tests. The definitions fall into one of the following categories: road, vehicle, crash and environmental.

\subsection{Road Definitions}

\subsubsection{Road}

The road consists of all travel lanes in the same direction of travel. This includes paved shoulders clear of obstacles. Roads may be divided or undivided.

\subsubsection{Undivided Road}

An undivided road is a road with two-way traffic (see Figure 1). Either double lane markers or a single dashed lane marker separates the directions of travel. A single dashed lane marker indicates passing in either direction. Double lane markers consist of combinations of solid and dashed stripes. Dashed indicates passing is permitted and solid prohibits passing.

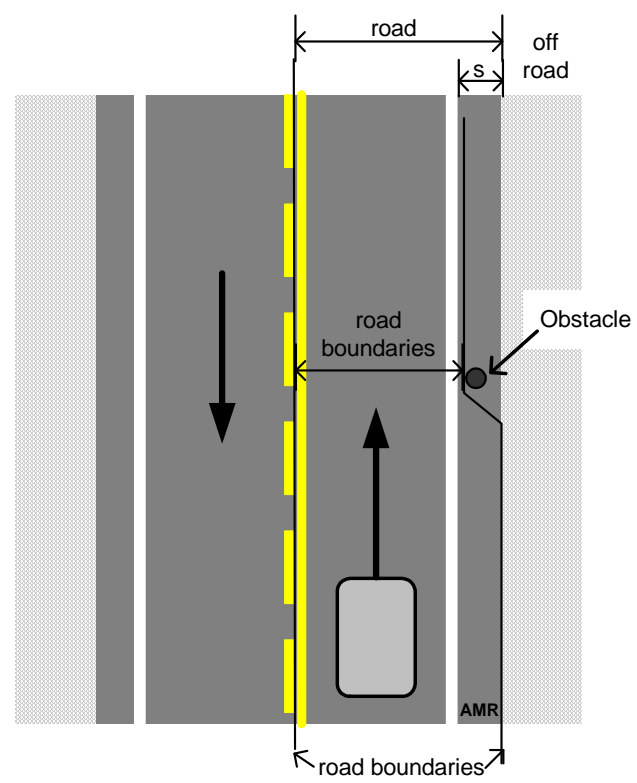

Figure 1. Undivided road includes all travel lanes and the shoulder (s)

\subsubsection{Divided Road}

A divided road is a road with one-way traffic (see Figure 2). Dashed white lane markers separate lanes of traffic. 


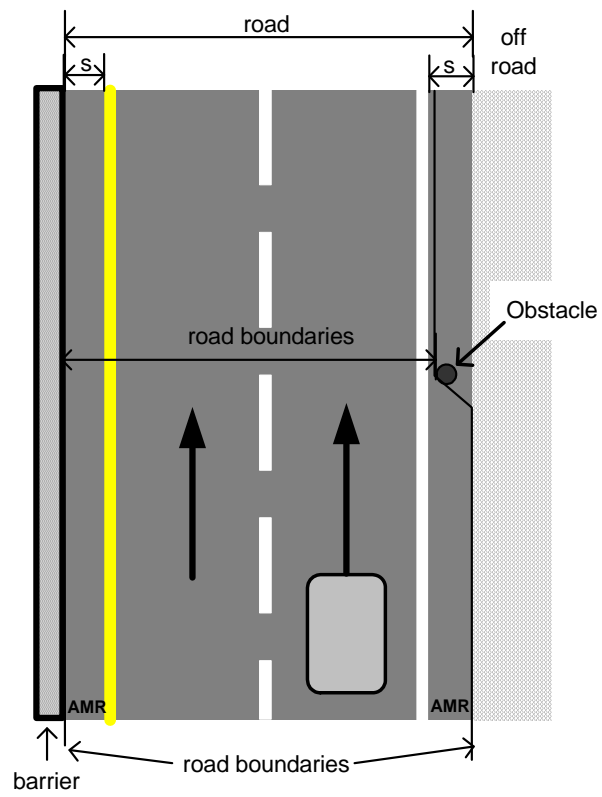

Figure 2. Divided road includes all travel lanes and the shoulder (s)

\subsubsection{Lane}

The lane is the area of paved surface between two parallel lane markings. The distance between the inside edges of the lane markings should be $3.7 \mathrm{~m}(12 \mathrm{ft})$. The lane must have markings on the left side. If the lane has no markings on the right side (worn or missing), then the lane is $3.7 \mathrm{~m}$ measured from the inside edge of the left side lane marker.

\subsubsection{Shoulder}

The shoulder is the portion of drivable surface to the right of an undivided road and on either side of a divided road. Refer to $s$ in Figure 1 and Figure 2.

\subsubsection{Available Maneuver Room}

The available maneuver room is the region of the road outside the outer lane marker that is safe to drive (see AMR in Figure 1 and Figure 2). Obstacles on the shoulder or absences of shoulders limit the AMR. By design, the AMR does not exceed a default maximum value $(1 \mathrm{~m})$, regardless of the size of the shoulder. The default minimum value is $15 \mathrm{~cm}(6 \mathrm{in})$.

\subsubsection{Road Boundary}

The road boundary (also referred to as road edge) is the real or imaginary boundary line on the pavement and defines the edge of the road (see Figure 1 and Figure 2). The road boundary is parallel to the outer lane marker and extends beyond the outer lane marker by the available maneuver room distance. A warning system senses the available maneuver 
room (e.g., obstacles on the shoulder) and adjusts the road boundary accordingly. The warning system also uses the distance to the road boundary to calculate when to issue a warning.

\subsubsection{Curved Road}

A curved road is a section of road with a radius less than $1000 \mathrm{~m}$.

\subsubsection{Straight Road}

A straight road is a section of road with a radius greater than $1000 \mathrm{~m}$.

\subsubsection{Lane Markings}

The definitions for lane markings come from part three of the Manual on Uniform Traffic

Control Devices (http://mutcd.fhwa.dot.gov/). Lane marker dimensions for the test are:

\begin{tabular}{|l|l|}
\hline Lane marker value & Dimensions \\
\hline Maker width & $10 \mathrm{~cm}$ to $15 \mathrm{~cm}(4$ in to $6 \mathrm{in})$ \\
\hline Dashed marker length & $3 \mathrm{~m}(10 \mathrm{ft}) \pm 5 \%$ \\
\hline $\begin{array}{l}\text { Dash marker gap length (distance between dashed } \\
\text { markers) }\end{array}$ & $9 \mathrm{~m}(30 \mathrm{ft}) \pm 5 \%$ \\
\hline
\end{tabular}

Appendix A "Methods for Determining Road Dimensions and Geometry" contains the recommended method to verify the lane marker dimensions.

The manual states:

Centerline pavement markings, when used, shall be the pavement markings used to delineate the separation of traffic lanes that have opposite directions of travel on a roadway and shall be yellow.

The centerline markings on two-lane, two-way roadways shall be one of the following as shown in Figure 3B-1:

A. Two-direction passing zone markings consisting of a normal broken yellow line where crossing the centerline markings for passing with care is permitted for traffic traveling in either direction;

B. One-direction no-passing zone markings consisting of a normal broken yellow line and a normal solid yellow line where crossing the centerline markings for passing with care is permitted for the traffic traveling adjacent to the broken line, but is prohibited for traffic traveling adjacent to the solid line; and

C. Two-direction no-passing zone markings consisting of two normal solid yellow lines where crossing the centerline markings for passing is prohibited for traffic traveling in either direction. 
Figure 3B-1. Examples of Two-Lane, Two-Way Marking Applications

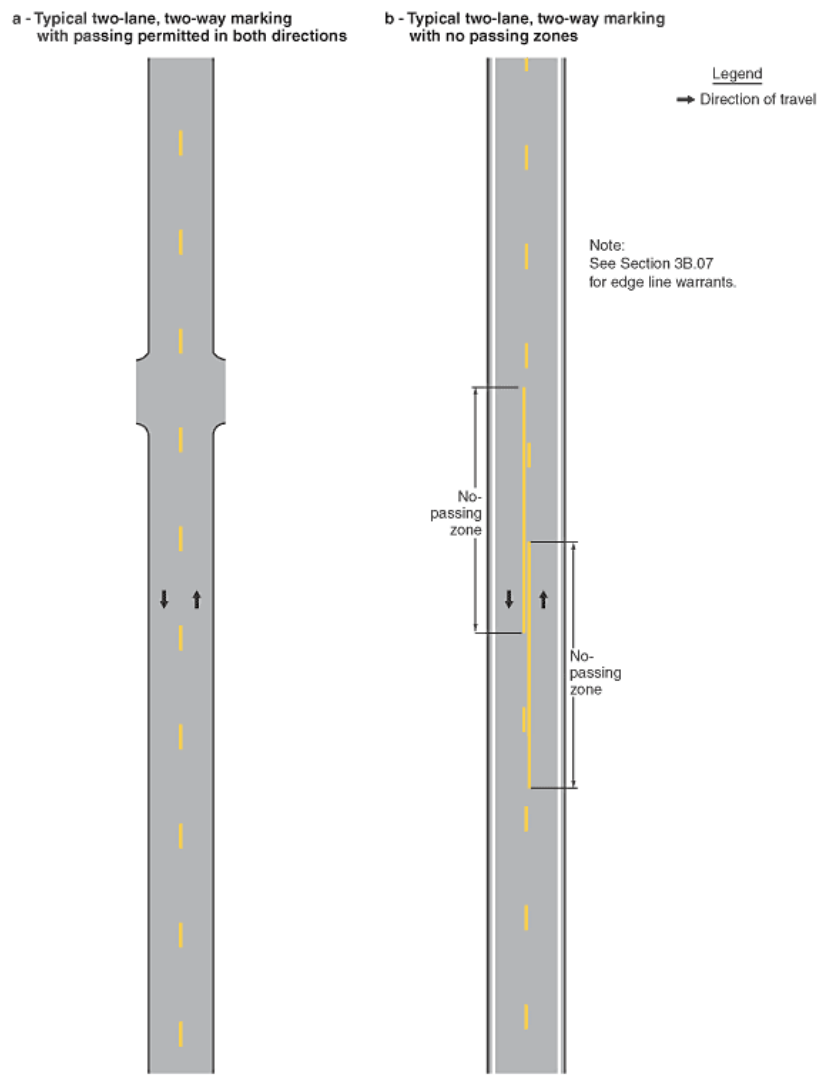

When used, lane line pavement markings delineating the separation of traffic lanes that have the same direction of travel shall be white.

Where crossing the lane line markings with care is permitted, the lane line markings shall consist of a normal broken white line.

Where crossing the lane line markings is discouraged, the lane line markings shall consist of a normal solid white line.

Where crossing the lane line markings is prohibited, the lane line markings shall consist of two normal solid white lines.

If used, the right edge line pavement markings shall consist of a normal solid white line to delineate the right edge of the roadway. 


\subsubsection{Lane Marker Reflectivity}

The lane marker reflectivity (or brightness) is the ratio between the luminance of the marker (light coming from the marker) and the illuminance on the marker (the light reaching the marker).

In daylight conditions, the measure of reflectivity is the coefficient of luminance under diffuse illumination $\left(\mathrm{Q}_{\mathrm{d}}\right)$.

In nighttime conditions, the brightness is a function of the headlights and the retroreflectivity of the lane marker. The measure of retroreflectivity is the coefficient of retroreflected luminance $\left(\mathrm{R}_{\mathrm{L}}\right)$.

Both of these coefficients are in units of millicandelas per square meter per lux (mcd $\bullet \mathrm{m}^{-}$ $\left.{ }^{2} \cdot 1 \mathrm{x}^{-1}\right)$. The geometry regarding the orientation of the light source, the orientation the viewer and the distance to the lane marker is part of the measurement process [9].

The values below come from Table 1 in reference [14].

\begin{tabular}{|c|c|}
\hline $\begin{array}{l}\text { Lane Marker } \\
\text { Color }\end{array}$ & $\begin{array}{l}\text { Minimum coefficient under diffuse } \\
\text { illumination, } Q_{\mathbf{d}}\left(\mathbf{m c d ~ m}^{-2} / \mathbf{l x}\right)\end{array}$ \\
\hline White & $\begin{array}{l}<130 \text { (for Asphalt) } \\
<160 \text { (for Concrete) }\end{array}$ \\
\hline Yellow & $<100$ \\
\hline
\end{tabular}

Appendix B contains the recommended method to verify the lane marker reflectivity.

\subsubsection{Lane Marker Color}

The daytime color of the lane marker is defined by its chromaticity and luminance $(\mathrm{x}, \mathrm{y}, \mathrm{Y})$. The CIE (Commission International d'Eclairage, or International Commission on Illumination, http://www.cie.co.at/cie/) chromaticity chart shows the coordinates (x,y) of visible colors. The luminance $(\mathrm{Y})$ defines the brightness of the color.

The four points listed in the table below bound the region of allowable colors for both white and yellow lane markers. Each color includes the luminance factor $(\mathrm{Y})$. The values come from Table 1 and Table 3 of reference [12] .

\begin{tabular}{|c|c|c|}
\hline $\begin{array}{c}\text { Lane Marker } \\
\text { Color }\end{array}$ & $\begin{array}{c}\text { Daytime Chromaticity } \\
\text { Coordinates (x,y) }\end{array}$ & Luminance factor Y (\%) \\
\hline White & $0.355,0.355$ & Minimum of 45 \\
& $0.305,0.305$ & \\
& $0.285,0.325$, & \\
\hline Yellow & $0.335,0.375$ & Minimum of 30 \\
& $0.560,0.440$ & \\
& $0.490,0.510$ & \\
& $0.420,0.440$, & \\
& $0.460,0.400$ & \\
\hline
\end{tabular}


A commercial measurement device should to measure color properties.

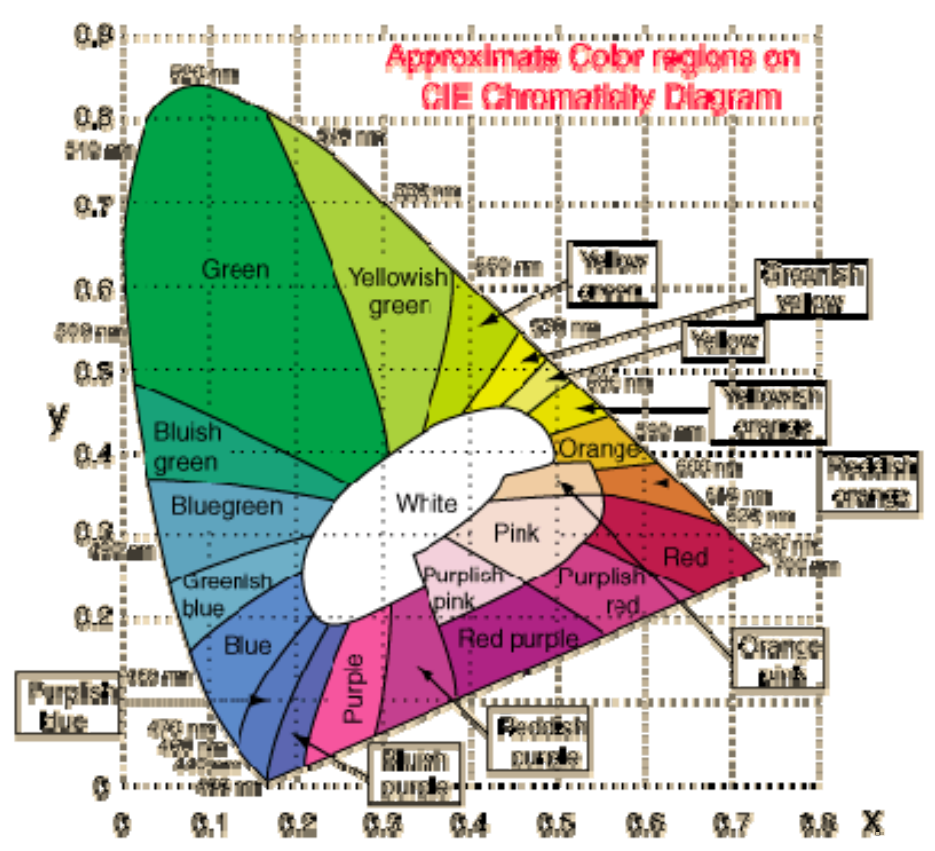

Figure 3. CIE Chromaticity Chart

\subsection{Vehicle Definitions}

Several definitions follow the terminology in reference [6].

\subsubsection{Vehicle Coordinate Systems}

Figure 4 shows the vehicle coordinate system. The origin of this system lies on the road directly under the vehicle's center of gravity. The z-axis points down into the road. 


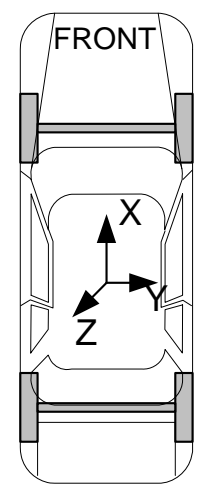

Figure 4. Vehicle coordinate system (z-axis is down)

\subsubsection{Longitudinal Speed}

The longitudinal speed is the vehicle's speed along the x-axis. The ROR report recommends that the warning system should determine vehicle velocity to an accuracy of 3 miles per hour (mph) (L-20 page 24 in [2]). Use the time to travel a known distance to verify the speedometer's accuracy.

The vehicle's speedometer provides vehicle speed during tests. An independent measurement system (IMS) verifies the vehicle's speed during a test using a dash camera aimed at the speedometer.

\subsubsection{Lateral Position}

The lateral position is the position of the vehicle's origin relative to the center of the lane. The lateral position measurement is along the vehicle y-axis. Most measurements in this test use a similar lateral measurement called Distance to Road Edge (see section 2.2.7).

\subsubsection{Lateral Velocity}

The lateral velocity is the rate of change of lateral position.

\subsubsection{Yaw Rate}

Yaw rate is the rate of rotation about the vehicle z-axis.

\subsubsection{Road Departure}

The vehicle departs the road when the outside edge of any of the vehicle tires crosses the outside edge of the road.

\subsubsection{Distance to Road Edge}

The distance to the road edge is the distance from the vehicle's outside front tire to the road edge measured along the vehicle's y-axis. 


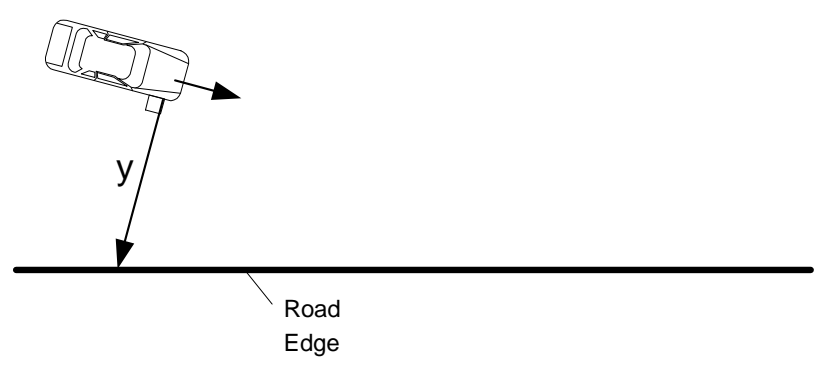

Figure 5. Distance to road edge is perpendicular to vehicle

The tests measure the lateral position using the IMS (see section 4) video. A calibration procedure transforms pixels in the image into distance to road-edge measurements. The analyst selects the pixel on the road edge and the IMS returns the distance.

\subsubsection{Road Departure Rate}

The road departure rate is the rate of change in distance to the road edge.

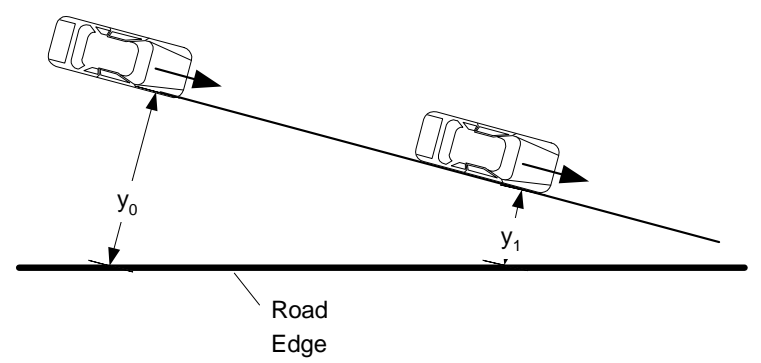

Figure 6. Computing lateral velocity

Use the following equation to compute road departure rate:

$v_{l a t}=\frac{y_{1}-y_{0}}{t_{1}-t_{0}}$

Road Departure Rate

Where:

$y_{1}, y_{0}-$ are the lateral positions at time $t_{l}$ and $t_{0}$ respectively (see figure above).

Many tests require the driver to depart the road at a given rate, for example, above 0.75 $\mathrm{m} / \mathrm{s}$. The driver achieves the desired departure rate by traversing a known distance in a known time interval. For example, the driver starts the departure at $0.75 \mathrm{~m}$ from the lane marker and crosses the lane marker in one second. Afterward, the analyst uses the IMS calibrated video to measure the exact departure rate. 


\subsubsection{Road Departure Angle}

The road departure angle is the angle between the forward $\mathrm{x}$-axis of the vehicle and the road edge.

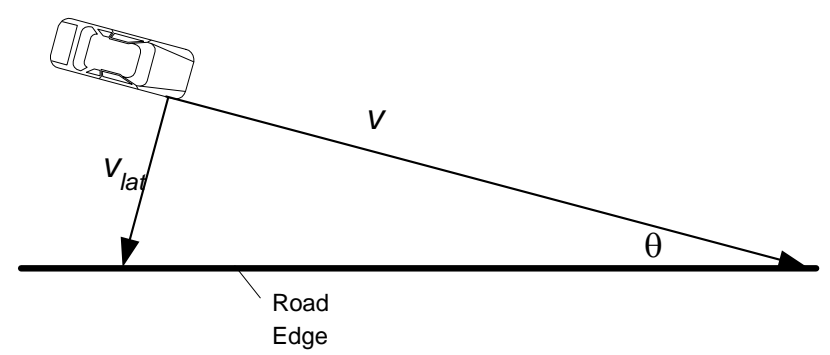

Figure 7. Departure angle on straight road. $V$ is the forward velocity and $V_{\text {lat }}$ is the lateral velocity.

The following formula estimates the departure angle from the departure rate and the forward velocity:

$$
\theta^{\circ}=\tan ^{-1}\left(\frac{v_{l a t}}{v}\right)\left(\frac{180}{\pi}\right) \quad \begin{aligned}
& \text { Departure Angle (in degrees) from forward and } \\
& \text { lateral velocities. }
\end{aligned}
$$

Where:

$v_{\text {lat }}=$ is the road departure rate $(\mathrm{m} / \mathrm{s})($ see section 2.2 .8$)$

$v=$ vehicle forward speed $(\mathrm{m} / \mathrm{s})$

\subsubsection{Distance to Curve Entry Point}

The curve entry point marks the transition between a straight road segment and a curved road segment (see Figure 8). The distance to the curve entry point is measured using the IMS in the following manner:

Determine the start location of the measurement, for example, the time of a warning. Note the lateral distance to the departure-side road edge. Record the initial $\left(n_{2}, e_{2}\right)$ location of the vehicle.

Advance the video until the lateral distance visibly decreases. This marks the curve entry point. Record the location of the vehicle at the curve entry point $\left(n_{2}, e_{2}\right)$.

Determine the distance to the curve entry point using the following formula:

$$
x_{m}=\sqrt{\left(n_{1}-n_{2}\right)^{2}+\left(e_{1}-e_{2}\right)^{2}}
$$

Measured distance to Curve Entry Point 


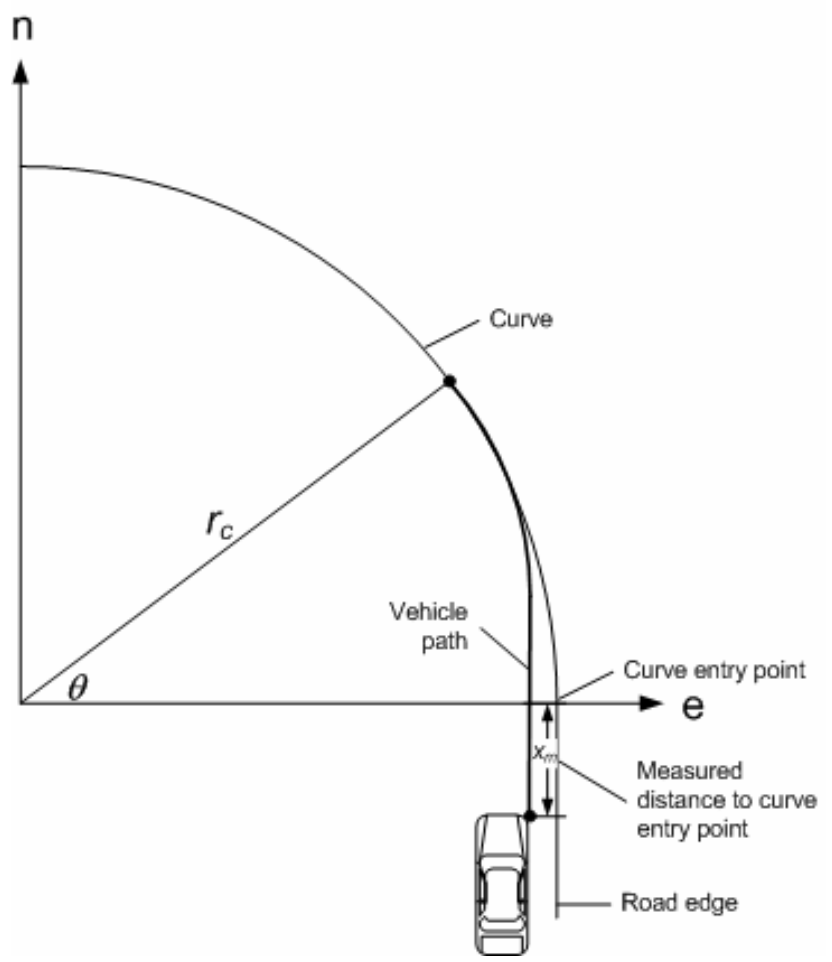

Figure 8. Measured distance to curve entry point at time of warning $\left(x_{m}\right)$

\subsection{Warning System Definitions}

\subsubsection{System Response Rating}

The rating of a system's response to a warning situation falls into one of the following categories [6]:

\begin{tabular}{|c|c|c|}
\hline System Response & $\begin{array}{c}\text { Situations Requiring a } \\
\text { Warning }\end{array}$ & $\begin{array}{c}\text { Situations in Which a } \\
\text { Warning is not Required }\end{array}$ \\
\hline Warning & $\begin{array}{c}\text { True Positive (TP) } \\
\text { Correctly warns of road } \\
\text { departure }\end{array}$ & $\begin{array}{c}\text { False Positive (FP) } \\
\text { False alarm }\end{array}$ \\
\hline No Warning & $\begin{array}{c}\text { False Negative (FN) } \\
\text { Misses road departure }\end{array}$ & $\begin{array}{c}\text { True Negative (TN) } \\
\text { Correctly does not warn of } \\
\text { road departure. }\end{array}$ \\
\hline
\end{tabular}

Table 2 Rating of warning system signals. 


\subsubsection{Imminent Crash Condition}

An imminent crash condition exists when there is a high probability of a lateral collision, a loss of steering control or a rollover. A RDCWS considers leaving the paved portion of the road at a high speed as an imminent crash condition because of the high probability of loss of steering control and rollover.

\subsubsection{Crash Warning}

A crash warning is a signal to the driver indicating an imminent crash condition exists and that the driver should take immediate action to prevent the crash.

\subsubsection{Advisory Warning}

An advisory warning is a signal to the driver that the vehicle is about to drift outside the current lane or that the current speed is too great for an upcoming curve.

\subsubsection{Warning Sensitivity}

Warning sensitivity is a user-controlled variable that determines the timing of a warning. High sensitivity produces earlier warnings and low sensitivities produce later warnings. The sensitivities affect evaluation of a warning system by setting a maximum value for lateral acceleration (due to driver steering correction) on a lateral drift departure and required longitudinal deceleration (due to driver braking to slow the vehicle) on a curve over-speed departure. A summary of the warning system sensitivities appears in the following table [7]. Unless stated otherwise, the sensitivity is at the mid-range value (3).

\begin{tabular}{|c|c|c|}
\hline Sensitivity & $\begin{array}{c}\boldsymbol{a}_{\text {lat }} \\
\mathrm{m} / \mathrm{s}^{2}\end{array}$ & $\begin{array}{c}\boldsymbol{d}_{\text {req }} \\
\mathrm{m} / \mathrm{s}^{2}\end{array}$ \\
\hline 1 & $4.12(0.42 \mathrm{~g})$ & $6.86(0.70 \mathrm{~g})$ \\
\hline 2 & $3.53(0.36 \mathrm{~g})$ & $5.88(0.60 \mathrm{~g})$ \\
\hline 3 & $2.94(0.30 \mathrm{~g})$ & $4.90(0.50 \mathrm{~g})$ \\
\hline 4 & $2.35(0.24 \mathrm{~g})$ & $3.92(0.40 \mathrm{~g})$ \\
\hline 5 & $1.76(0.18 \mathrm{~g})$ & $2.94(0.30 \mathrm{~g})$ \\
\hline
\end{tabular}

Table 3 Warning system sensitivity values for lateral acceleration $\left(a_{l a t}\right)$ and longitudinal deceleration $\left(d_{r e q}\right)$, where g equals gravity $\left(9.8 \mathrm{~m} / \mathrm{s}^{2}\right)$.

\subsubsection{Reaction Time}

The reaction time defines the amount of delay between onset of a warning and onset of a steering maneuver by the driver. An average reaction time for these tests is $t_{r}=1.5 \mathrm{~s}$. A lower bound $\left(t_{l}\right)$ and an upper $\left(t_{h}\right)$ define the range of acceptable reaction times. The evaluation equations use these values to determine the earliest and latest location of a warning. Table 4 gives suggested values for the reaction times. 


\begin{tabular}{|l|l|l|}
\hline Name & Time & Description \\
\hline$t_{l}$ & $0.75 \mathrm{~s}$ & $\begin{array}{l}\text { Lower bound used to define latest possible } \\
\text { warning location. }\end{array}$ \\
\hline$t_{r}$ & $1.5 \mathrm{~s}$ & Ideal reaction time \\
\hline$t_{h}$ & $2.0 \mathrm{~s}$ & $\begin{array}{l}\text { Upper bound used to define earliest } \\
\text { possible warning location. }\end{array}$ \\
\hline
\end{tabular}

Table 4. Reaction time with low and high limits.

\subsubsection{Obstacle}

An obstacle is an object of sufficient mass to cause damage to a vehicle upon collision.

\subsubsection{Radar Corner Cube Reflector}

Radar cross section (RCS) is the measure of a target's ability to reflect radar signals in the direction of the radar receiver. The corner reflector has an RCS almost as high as a flat plate but over a wider reflectance angle (over $\pm 60^{\circ}$ ).

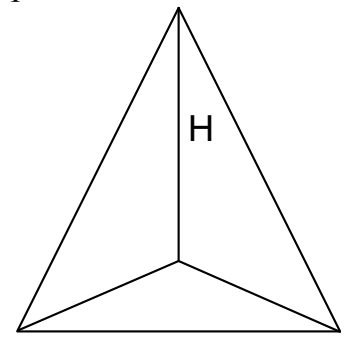

Figure 9. Corner Cube Reflector

Use the following equation to determine the height of a corner cube for a desired RCS.

$H=\left(\frac{3 \lambda^{2} \sigma}{4 \pi}\right)^{1 / 4}$

Height of corner cube

Where:

$\mathrm{H}=$ height of corner cube $(\mathrm{m})$

$\sigma=$ desired radar cross section (RCS) $\left(\mathrm{m}^{2}\right)$

$\lambda=$ radar wavelength $(\mathrm{m})$

\subsubsection{Time of Warning}

The time of warning is the time when the warning system issues an alert. Determining whether a warning is late, early or on time requires the time of the warning relative to the road departure.

The IMS (see section 4) captures the time of the warning from the visual icon on the warning system display or from the audible alert (using a microphone). The analyst searches through the video to locate the warning and uses the GPS clock and the video frame clock (the frames are $29.9 \mathrm{~ms}$ apart) to measure the time of the warning. 


\subsubsection{Time to Line Crossing}

The time to line crossing is the calculated time until the vehicle's lead tire crosses an inpath lane marker given the vehicle continues on its current trajectory.

\subsubsection{Time to Road Departure}

The time to departure is the time until the vehicle's lead tire crosses a line parallel to the outer lane marker offset by the available maneuver room.

\subsubsection{Nuisance Alarm}

Nuisance alarms are warnings deemed unwarranted by a driver. They include false alarms due to incorrectly predicting the time or position of a crash (i.e., early warning) and false positives due to incorrect sensor readings (from noisy or cluttered environments).

\subsection{Environmental Definitions}

\subsubsection{Daylight Time}

Daylight is the times of day starting at sunrise and ending at sunset. The times are available from the US Naval Observatory (http://aa.usno.navy.mil/data/).

\subsubsection{Night Time}

Night is the times of day after civil twilight at sunset and ending at twilight before sunrise. Civil twilight is when the sun is 6 degrees below the horizon. According to definitions used by the US Naval Observatory:

In the morning before the beginning of civil twilight and in the evening after the end of civil twilight, artificial illumination is normally required to carry on ordinary outdoor activities. Complete darkness, however, ends sometime prior to the beginning of morning civil twilight and begins sometime after the end of evening civil twilight.

The times of civil twilight are available from the US Naval Observatory (http://aa.usno.navy.mil/data/).

\subsubsection{Rain}

Rain is precipitation in the form of water drops larger than $0.5 \mathrm{~mm}$ (0.02 in). The following table shows how rainfall intensity is determined:

\begin{tabular}{|c|l|l|}
\hline $\begin{array}{c}\text { Rainfall } \\
\text { Intensity }\end{array}$ & Rate-of-fall in 6 min & \multicolumn{1}{c|}{ Visual Estimation } \\
\hline Light & $\begin{array}{l}\leq 0.25 \mathrm{~mm} \\
(0.01 \mathrm{in})\end{array}$ & $\begin{array}{l}\text { From scattered drops that, regardless of } \\
\text { duration, do not completely wet an exposed } \\
\text { surface up to a condition where individual } \\
\text { drops are easily seen }\end{array}$ \\
\hline Moderate & $0.25 \mathrm{~mm}$ to & From scattered drops that, regardless of \\
\hline
\end{tabular}




\begin{tabular}{|c|l|l|}
\hline & $0.762 \mathrm{~mm}(0.03 \mathrm{in})$ & $\begin{array}{l}\text { duration, do not completely wet an exposed } \\
\text { surface up to a condition where individual } \\
\text { drops are easily seen. }\end{array}$ \\
\hline Heavy & $>0.762 \mathrm{~mm}$ & $\begin{array}{l}\text { Rain seemingly falls in sheets; individual drops } \\
\text { are not identifiable; heavy spray to the height } \\
\text { of several centimeters over hard surfaces. }\end{array}$ \\
\hline
\end{tabular}

Table 5. Rain rate of fall.

\subsubsection{Fog}

Fog is as a visible aggregate of minute water particles (droplets), which is close to the Earth's surface and reduces horizontal visibility to less than $1 \mathrm{~km}(5 / 8 \mathrm{mi})$, and unlike drizzle, it does not fall to the ground.

Names given to fog types identify their methods of formation. The principle types are radiational fog, ice fog, advection fog, upslope fog, rain-induced fog, and steam fog. These types of fog are called "dense" when the surface visibility is equal to or less than $400 \mathrm{~m}$ (1/4 mi). A Dense Fog Advisory occurs when the dense fog becomes widespread.

\subsubsection{Snow}

Snow precipitation consists of water crystals, mostly branched in the form of six-pointed stars. It usually falls steadily for several hours or more. Qualifiers, such as occasional or intermittent, indicate a steady, prolonged (for several hours or more) fall is not expected. Like rain, visibility determines snow intensity. The following table shows snow intensity versus visibility:

\begin{tabular}{|l|l|}
\hline \multicolumn{1}{|c|}{ Snow Intensity } & \multicolumn{1}{c|}{ Visibility } \\
\hline Light & $>1 \mathrm{~km}(5 / 8 \mathrm{mi})$ \\
\hline Moderate & $400 \mathrm{~m}(1 / 4 \mathrm{mi})$ to $1 \mathrm{~km}$ \\
\hline Heavy & $<400 \mathrm{~m}$ \\
\hline
\end{tabular}




\section{Basic Test Organization}

Each test procedure in this document consists of the following subsections.

\section{Test Title}

Each test in this document starts with a short description of the purpose of the test and a figure (see Figure 10) showing the trajectory of the vehicle, the road geometry, pertinent test props and key events of the test.

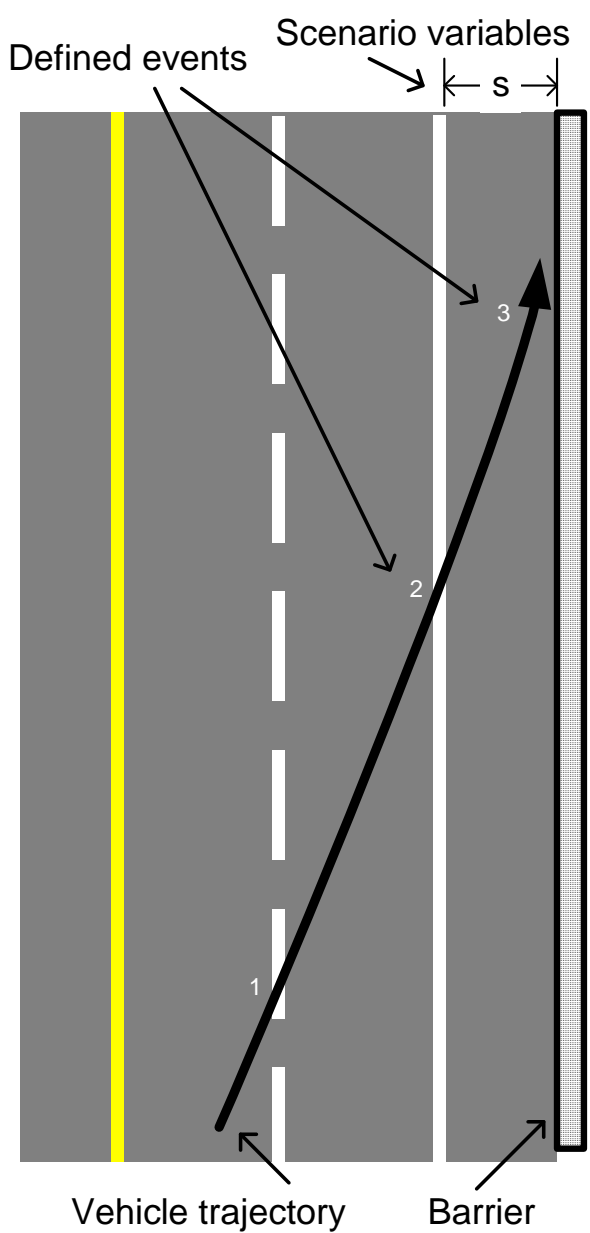

Figure 10. Each test procedure includes a figure that illustrates pertinent aspects of the test.

\section{Events and System Response}

This section describes the events during the test and the response of the warning system to the event. Typically, the response is a specific warning signal. 


\section{Conditions}

This section describes the conditions for the test. Some conditions remain fixed throughout the test, for example, road geometry and lane marker quality. Other conditions may vary between a set of tests, for example, speed and departure rate.

\section{Measurements}

This section lists all the measurements that are required for the tests. Also presented are methods and procedures for processing the measurements.

\section{Procedure}

This section lists the steps of the test, which have the following format:

1. Setup and verify scenario conditions.

2. Drive system/vehicle along desired trajectory

3. Record trajectory and warning system responses

4. Process data to determine performance variables

5. Evaluate the system response to determine whether system passed

\section{Evaluation Methods}

This section contains the methods for judging whether the system passes the test. The methods used for most tests are:

1. Is the correct warning system response given (e.g., did the system miss the event)?

2. Is the timing of the warning acceptable? 


\section{Independent Measurement System}

This section describes an independent measurement system used to collect warningsystem performance data required for system evaluation. Evaluation of the warning system should use sensors and equipment separate from the warning system under test. For example, the distance to a lane marker at the time of warning should not come from the warning system itself. Errors in the warning system camera and image-processing algorithm could lead to incorrect assessment of warning system performance. Details of the IMS appear in [5]. This section provides enough details to understand one approach for collecting data and measurements to analyze warning system performance.

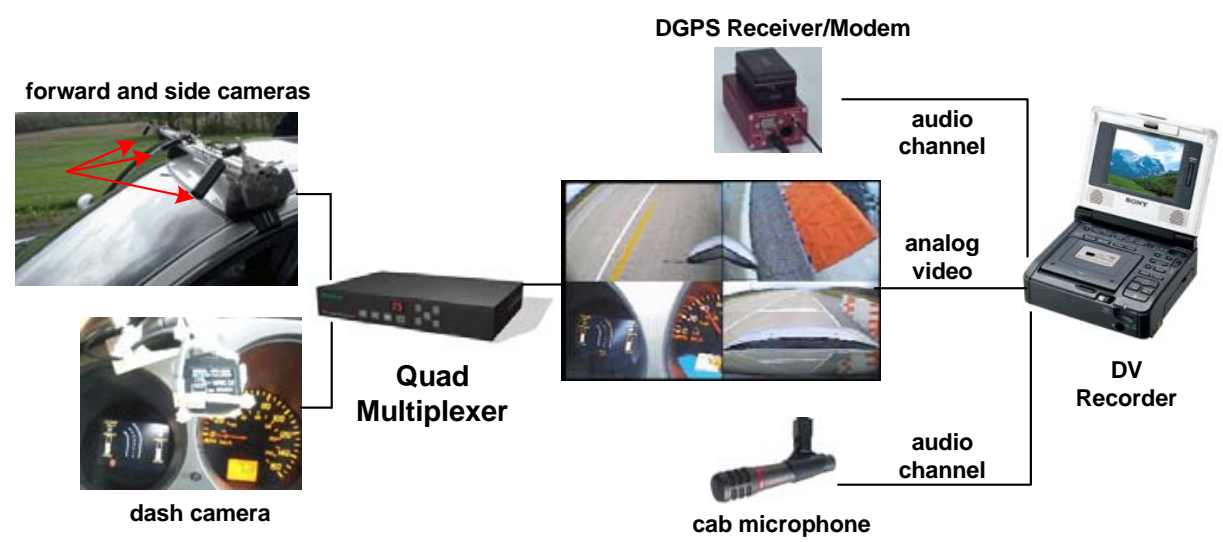

Figure 11. Independent Measurement System

Figure 11 illustrates the components of the IMS described [5]. Three cameras situated on a roof rack provide forward and side-looking video. A fourth camera mounted on the dash captures the warning system display. A quad multiplexer combines the four video signals into a single video stream. The output of the quad appears in Figure 12. 


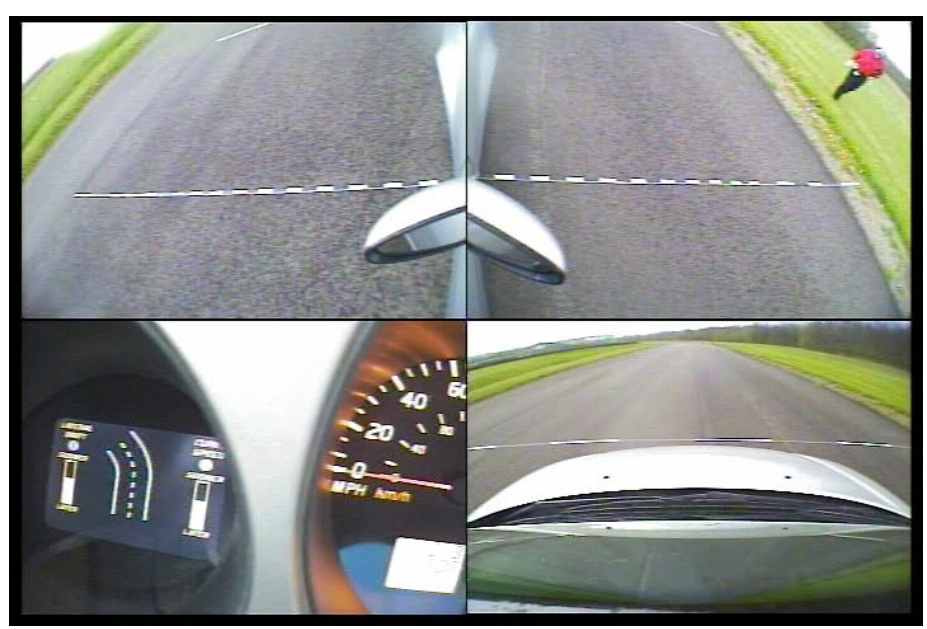

Figure 12. Four camera views combined into one. Top left and right are view of calibration sticks aligned with front wheels. Bottom right shows calibration sticks on the road in front of vehicle. Bottom left is view of warning system display.

The cameras perform the following functions:

1. The left and right downward facing cameras measure lateral distances relative to the front wheels. The top left and right quad in Figure 12 show calibration sticks for calibrating the side looking cameras. The black and white stripes on the calibration sticks have known dimensions. The user identifies the pixel locations of the stripes (see red circles in top left of Figure 13, which defines a transformation between pixel coordinates in the image and ground coordinates relative to the front wheels. After camera calibration, the sticks are no longer required. The overlaid red circles indicate the calibrated portion of the video data (see top right quad in Figure 13). Clicking on a pixel centered with the red circle returns the lateral distance as if the calibrated sticks existed. Linear interpolation gives distance measurements between the circles.

2. A forward facing camera provides lateral measurements forward of the vehicle. The camera calibration uses calibration sticks as well. This view provides a better view of the surrounding environment then just the side looking cameras.

3. A dash view camera shows vehicle speed and when an alert is given. 


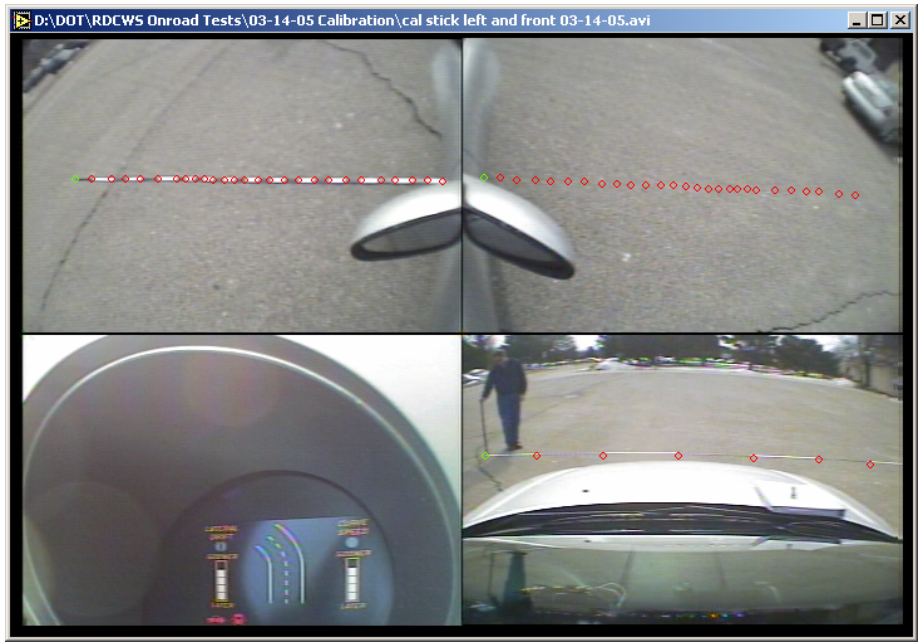

Figure 13. Result of pixel to ground calibration is shown using red circles. Circles in top left view identify transitions from black to white marking on calibration stick. After removal of the calibration stick (e.g., top right view), the circles provide a reference for the operator when measuring lateral distances from the front wheel.

Other components of the IMS include a Differential Global Positioning Satellite (DGPS) receiver to sense vehicle position and velocity, and a cab microphone to pick up the audible alerts and comments from the driver. To simplify data collection, a modem-like device converts the GPS data into an audible signal. A digital videocassette recorder (DV VCR) simultaneously records the output of the video, microphone (one audio channel) and GPS (the second audio channel), ensuring synchronization of the data streams. 


\section{Warning System Evaluations}

This section describes various methods for rating warning system performance that are common among tests. The methods describe how to score the system, but the methods do not specify what score is acceptable. Some example criteria for establishing an acceptable score include:

1. The system must pass $95 \%$ of the runs for a given test (good when there is large number of test runs)

2. The system may only fail 1 time for a given set of test runs (good when there is a small number of test runs)

The organization interested in the test results should specify the acceptable pass/fail criterion.

\subsection{Lateral Drift Evaluation}

A warning must provide a driver with time to react and time to avoid (i.e., steer away from) a departure. The reaction phase starts at the time of warning and continues until the driver initiates the steering correction. The steer phase consists of the trajectory the vehicle follows during the steering correction. The magnitude of the steer should not exceed $a_{\text {lat }}$ limits defined in Table 3 of section 2.3.5 (Warning Sensitivity). The lateral drift analysis uses separate equations for straight or curved roads.

\subsubsection{Straight Road}

A lateral drift on a straight road occurs when the vehicle departs a straight road at a fixed angle to the direction of the roadway.

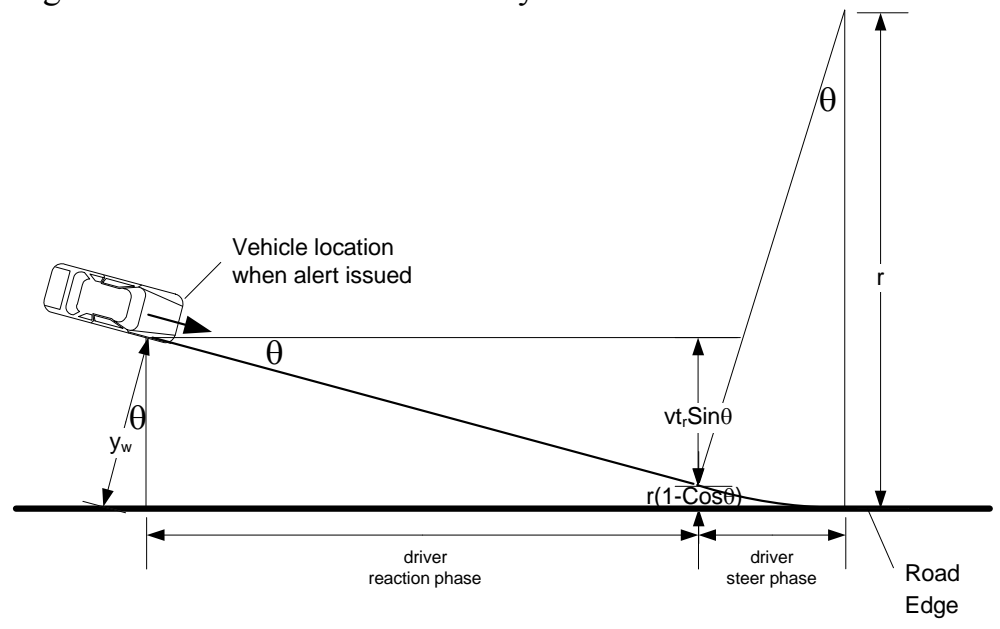

Figure 14. Geometry for a lateral drift scenario on a straight road

Given the geometry of the lateral departure of a straight road shown in Figure 14, one determines the desired lateral distance to issue an alert using the following equation: 
$y_{w}=\frac{v t_{r} \sin \theta+r(1-\cos \theta)}{\cos \theta}$,

Where:

$v=$ vehicle forward speed $(\mathrm{m} / \mathrm{s})$

$t_{r}=$ delay between warning and start of steering (see section 2.3.6) (s).

$\theta=$ the departure angle (radians). If measuring departure rate, convert to angle using equation in section 2.2.9).

$r=$ the radius of the curve the vehicle must travel to avoid the departure (m)

The equation simplifies to:

$y_{w}=v t_{r} \tan \theta+r(1 / \cos \theta-1)$.

Use the measured lateral velocity $\left(v_{\text {lat }}\right)$ to calculate the departure angle (see section 2.2.9):

$\theta=\tan ^{-1}\left(\frac{v_{\text {lat }}}{v}\right)$

The radius should not cause the vehicle to exceed a maximum lateral acceleration ( $a_{\text {lat }}$, see section 2.3.5), therefore:

$r=\frac{v^{2}}{a_{\text {lat }}}$

To determine the desired location of a warning (the lateral distance to road boundary) for comparison with the measured time of a warning:

1. Measure $v$ and $v_{\text {lat }}$ at the time of warning.

2. Obtain $t_{r}$ from section 2.3 .6 and $a_{\text {lat }}$ from section 2.3 .5 sensitivity setting 3 .

3. Calculate desired location using the following equation:

$y_{w}=v_{\text {lat }} t_{r}+\frac{v^{2}}{a_{\text {lat }}}(1 / \cos \theta-1)$

Use the following procedure to determine whether the actual measured warning location during a test is early, late or on time.

1. Use the desired location warning equation to determine the latest warning location $(L W L)$ (warning occurs closer to road boundary) using sensitivity setting 1 (highest $a_{\text {lat }}$ from section 2.3.5) and reaction time $t_{l}$ (shortest reaction time from section 2.3.6).

2. Use the desired location warning equation to determine the earliest warning location $(E W L)$ (warning occurs farther from road boundary) using sensitivity setting 5 (lowest $a_{\text {lat }}$ from section 2.3.5) and reaction time $t_{h}$ (longest reaction time from section 2.3.6). Use the following relationships to compare the measured location of the warning $\left(y_{m}\right)$ with $L W L$ and $E W L$ to determine whether the warning is early, late or on time (refer to Figure 16):

If $y_{m}>E W L$, then the warning is early

If $y_{m}<L W L, \quad$ then the warning is late

If $E W L \geq y_{m} \leq L W L$, then the warning is on time 


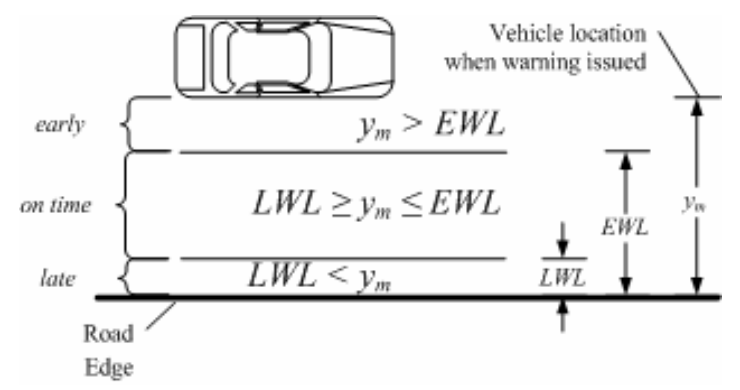

Figure 15 Relationship to determine timeliness of warning on lateral-distance based warning.

\subsubsection{Curved Road}

A lateral drift on a curved road occurs when the vehicle is traveling straight and departs a curved road.

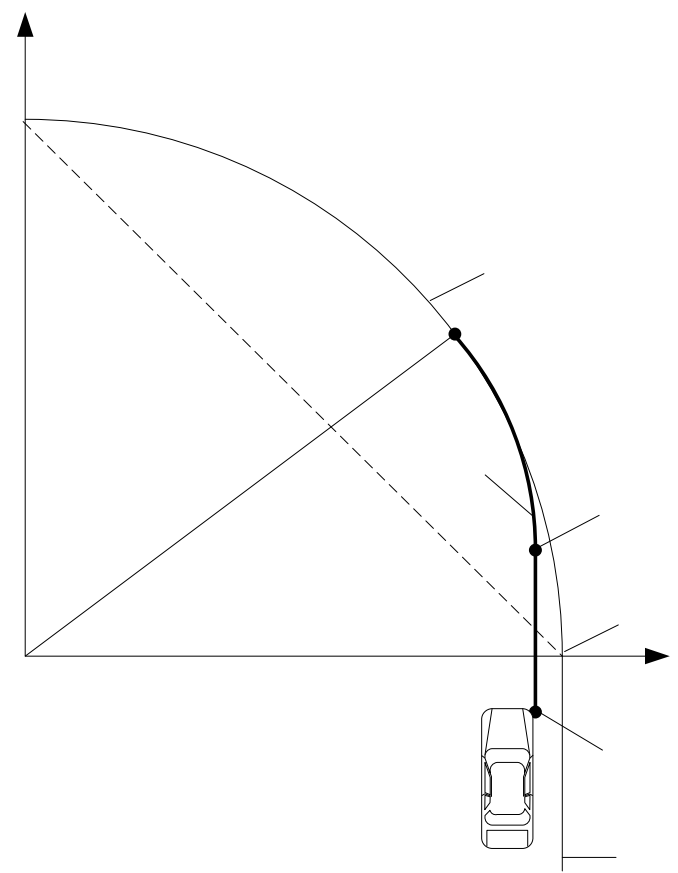

Figure 16. Geometry for a lateral drift scenario on a curved road

Reference [10] describes the derivation of the lateral acceleration required to avoid a lateral departure on a curved road (see Figure 16 - note the change in $\mathrm{x}$ and $\mathrm{y}$ axes to remain consistent with y measurements being lateral from the vehicle): 


$$
a_{\text {lat }}=\frac{v^{2}}{r_{c}-\frac{x_{w}+v t_{r}}{\sin \left(2 \tan ^{-1}\left(\frac{r-y_{w}}{x_{w}+v t_{r}}\right)\right)}}
$$

Where:

$v=$ vehicle forward speed $(\mathrm{m} / \mathrm{s})$

$r_{c}=$ the radius of the curve $(\mathrm{m})$

$t_{r}=$ delay required by driver to initiate the steering correction

$y_{w}=$ the lateral distance to the center of the curve (m).

$x_{w}=$ the longitudinal distance to the curve entry point (m) (See section 2.2.10.)

Subject to:

$v t_{r}<\sqrt{r_{c}^{2}-y_{w}^{2}}-x_{w}$

Formatted: Lowered by $7 \mathrm{pt}$

(The onset of steering must occur prior to the road boundary).

Note that in this scenario, the vehicle must travel straight during the departure (i.e., remain parallel to the road edge until entering the curve). The initial lateral position $\left(y_{w}\right)$ dictates the ideal location for a warning $\left(x_{w},\right)$, which is relative to the curve entry point. The curve entry point is the point where the lateral distance to the road edge starts to decrease. Using the IMS, the distance is derived from speed and time.

To determine the desired location of a warning (longitudinal distance to curve entry) for comparison with the measured time of a warning:

1. Measure $v$, the lateral distance to the road edge $y_{m}$, and $r_{c}$ during a test.

2. Determine $y_{w}$ from $y_{w}=r_{c}-y_{m}$.

3. Obtain $t_{r}$ from section 2.3.6 and $a_{\text {lat }}$ from section 2.3 .5 sensitivity setting 3 .

4. Calculate desired location using the following equation:

$$
x_{w}=\sqrt{\left(r_{c}-y_{w}\right)\left(r_{c}-\frac{2 v^{2}}{a_{\text {lat }}}+y_{w}\right)}-v t_{r}
$$

Use the following procedure to determine whether the actual measured warning location during a test is early, late or on time.

1. Measure $v$, the lateral distance to the road edge $y_{m}$, the longitudinal distance to the curve entry $x_{m}$ (note: $x_{m}$ is negative if vehicle hasn't entered the curve) and $r_{c}$ at the time of a warning.

2. Determine $y_{w}$ and $x_{w}$ from $y_{w}=r_{c}-y_{m}$ and $x_{w}=x_{m}$.

3. If $v t_{r} \geq \sqrt{r_{c}^{2}-y_{w}^{2}}-x_{w}$ then turn begins after vehicle leaves the lane (can't use equation (2)). Classify warning as late.

4. Use equation (2) to determine the lateral acceleration required to stay on the road.

5. If $a_{\text {lat }}>$ sensitivity setting 1 (highest $a_{\text {lat }}$ from section 2.3 .5 ) then classify warning as late.

6. If $a_{\text {lat }}<$ sensitivity setting 5 (lowest $a_{\text {lat }}$ from section 2.3 .5 ) then classify warning as early. 
7. Else $a_{\text {lat }}$ falls within the acceptable limits and classify the warning as on time.

\subsection{Curve Speed Evaluation}

A curve-speed warning must provide a driver with time to react (reaction phase) and to slow (brake phase) to a safe speed. The braking action should not exceed a limit on deceleration.

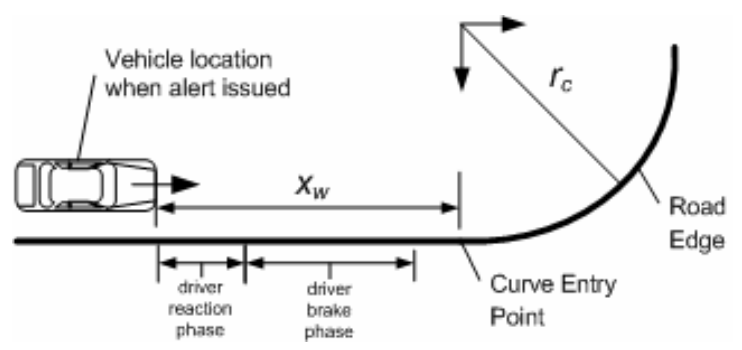

Figure 17. Geometry for a curve speed warning scenario.

Use the following equation to calculate the amount of deceleration required to reach the safe speed:

$d_{r e q}=\frac{v^{2}-v_{s}^{2}}{2\left(x_{w}-t_{r} v\right)}$

Where:

$d_{r e q}=$ required deceleration $\left(\mathrm{m} / \mathrm{s}^{2}\right)$

$v=$ vehicle initial forward speed $(\mathrm{m} / \mathrm{s})$

$v_{s}=$ safe speed for the curve $(\mathrm{m} / \mathrm{s})$

$t_{r}=$ driver reaction time (s)

$x_{w}=$ the distance between the warning location and the start of the curve (m) (See section 2.2.10.)

The safe speed for a curve of radius $r$ does not exceed a maximum lateral acceleration $\left(a_{\text {lat }}\right.$, see section 2.3.5). Use the following equation to calculate the safe speed:

$v_{s}=\sqrt{a_{l a t} r}$

To determine the desired location of a warning (distance to curve entry) for comparison with the measured time of a warning:

1. Measure $v, y_{a}$, and $r_{c}$ during a test.

2. Obtain $t_{r}$ from section 2.3.6, and $a_{\text {lat }}$ and $d_{r e q}$ from section 2.3 .5 sensitivity setting 3 .

3. Calculate desired location using the following equation:

$$
x_{w}=\frac{v^{2}-v_{s}^{2}}{2 d_{r e q}}+t_{r} v
$$

Use the following procedure to determine whether the actual measured warning location during a test is early, late or on time. 
1. Use the desired location warning equation to determine the latest warning location ( $L W L$ ) (warning occurs closer to road boundary) using sensitivity setting 1 (highest $a_{\text {lat }}$ from section 2.3.5) and reaction time $t_{l}$ (shortest reaction time from section 2.3.6).

2. Use the desired location warning equation to determine the earliest warning location $(E W L)$ (warning occurs farther from road boundary) using sensitivity setting 5 (lowest $a_{\text {lat }}$ from section 2.3.5) and reaction time $t_{h}$ (longest reaction time from section 2.3.6).

3. Use the following relationships to compare the measured location of the warning $\left(x_{m}\right)$ with $L W L$ and $E W L$ to determine whether the warning is early, late or on time (refer to Figure 18):

If $x_{m}>E W L, \quad$ then the warning is early

If $x_{m}<L W L, \quad$ then the warning is late

If $E W L \geq x_{m} \leq L W L$, then the warning is on time

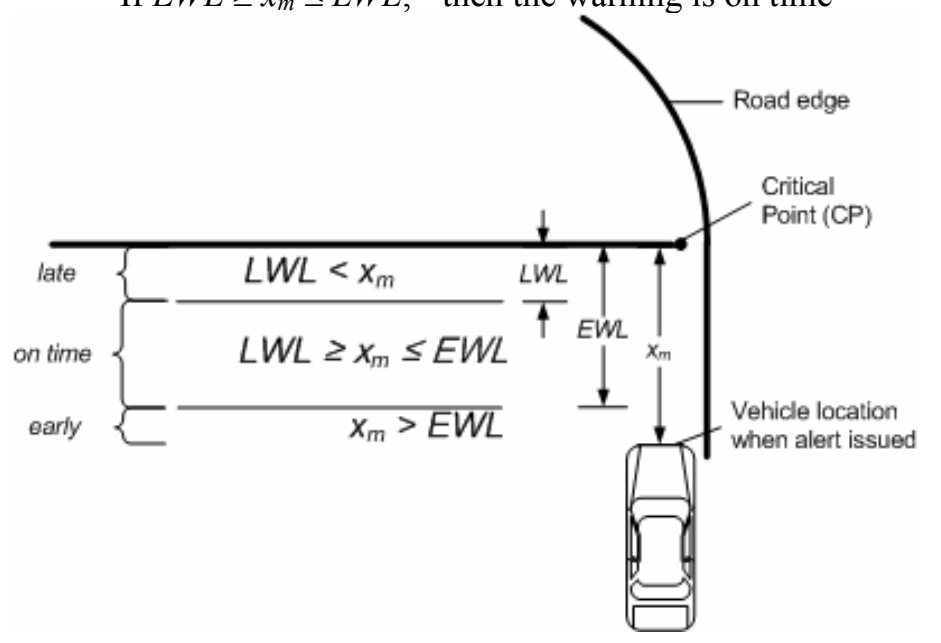

Figure 18. Relationship to determine timeliness of warning on lateral-distance based warning.

\subsection{Timeliness of Warning}

Once warnings are evaluated as early, late or on time for the given scenario (departure on straight road, departure on curved road, curve speed departure), the results are accumulated as follows:

$\begin{array}{ll}\left(\frac{\# \text { Early }}{\# \text { TP }}\right) \cdot 100 & \text { Percent of Warnings Early } \\ \left(\frac{\# \text { OnTime }}{\# \text { TP }}\right) \cdot 100 & \text { Percent of Warnings on Time } \\ \left(\frac{\# \text { Late }}{\# \text { TP }}\right) \cdot 100 & \text { Percent of Warnings Late }\end{array}$


Where \#Early, \#OnTime, \#Late, and \#TP are the total number of early warnings, on time warnings, late warnings, and the true positive warnings. Each test procedure describes how to determine if a warning is a true positive (also referred to as the correct response $(\mathrm{CR})$ ). The results of several test runs are summarized in the following table.

Test Run Summary Results

\begin{tabular}{|c|c|c|c|c|c|c|c|c|}
\hline $\begin{array}{c}\text { Combined Results for } \\
\text { all runs }\end{array}$ & $\begin{array}{c}\# \text { of } \\
\text { runs }\end{array}$ & $\begin{array}{c}\# \\
\text { CR }\end{array}$ & $\begin{array}{c}\# \\
\text { OT }\end{array}$ & $\begin{array}{c}\# \\
\text { E }\end{array}$ & $\begin{array}{l}\# \\
\text { L }\end{array}$ & OT & E & \% \\
\hline Date/time of test runs & & & & & & & & \\
\hline
\end{tabular}

\subsection{Efficacy Rate}

Reference [6] defines the efficacy rate as the rate that a desired effect occurs.

$$
\text { efficacy rate }=\left(\frac{\# T P}{\# T P+\# F N}\right) \cdot 100
$$

\subsection{False Alarm Rate}

Reference [6] defines the false alarm rate.

$$
\text { false alarm rate }=\left(\frac{\# F P}{\# T P+\# F P}\right) \cdot 100
$$




\section{Tests for Departure on Roads with Default Minimum AMR}

The tests in this section evaluate warning system response to road departures where the available maneuver room is at a default minimum. This situation arises when the sensor processing has unacceptable uncertainty, when maps of the road network are unavailable, or when the warning system lacks the sensing capability to detect the drivable surface on the side of the road.

The tests evaluate the system's ability to:

1. Detect lane markers,

2. Determine lane marker type,

3. Determine departure velocities,

4. Determine driver lane change intent

The test scenarios include departures on a divided straight road, on an undivided straight road and on a divided curved road. 


\subsection{One-Way Straight Road (Default Minimum AMR)}

This test assesses the performance of the lateral drift warning function for road departures on divided straight roads. This scenario falls under the "going straight on freeway with two lanes at non-junction" scenario identified in the NHTSA test scenario report as one of the major settings for road departure crashes [3]. The test also examines warning system response during lane changes (i.e., when vehicle crosses dashed lane markers) with and without turn signals.

The figure below illustrates the test scenario. Both a left and a right departure are tested.

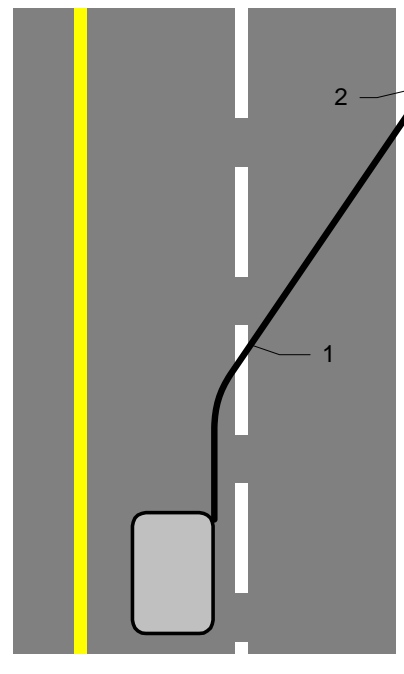

A

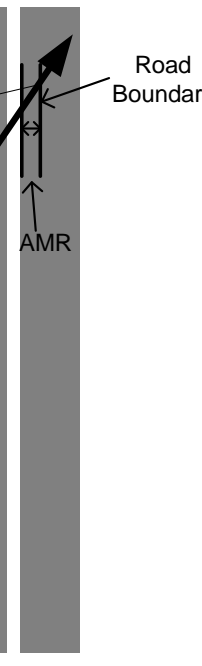

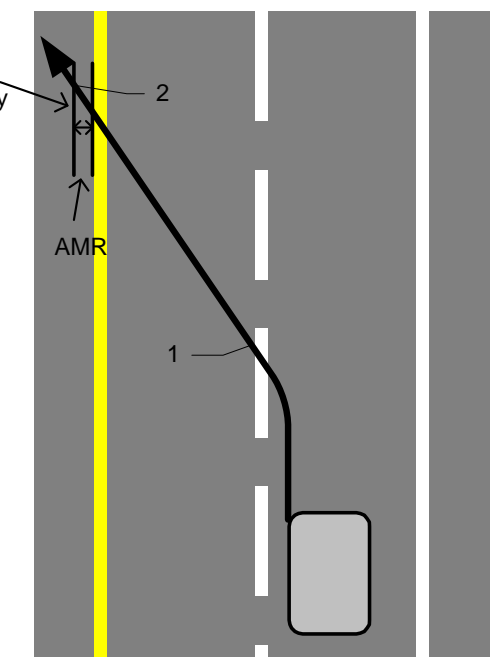

B

Figure 19. Scenario for road departure on divided road

\subsubsection{Events and System Response}

The following table defines the events for this test and the correct warning signal the system shall provide. See the figure above to locate the event (e.g., Id A1 is label 1 in figure A). 


\begin{tabular}{|c|l|l|}
\hline Id & \multicolumn{1}{|c|}{ Event } & \multicolumn{1}{c|}{ Correct Response } \\
\hline A1 & Crossed dashed line & $\begin{array}{l}\text { 1. Issue no warning if correct turn signal active. } \\
\text { 2. Issue an advisory warning if no turn signal active } \\
\text { or incorrect turn signal active. }\end{array}$ \\
\hline A2 & Road departure & 1. Issue road departure warning \\
\hline B1 & Same as A1 & 1. Same as A1 \\
\hline B2 & Same as A2 & 1. Same as A2 \\
\hline
\end{tabular}

\subsubsection{Test Conditions}

The conditions for this test include the following.

\subsubsection{Road Geometry}

The road geometry for the test is the following:

\begin{tabular}{|l|l|}
\hline Road Geometry & Dimension \\
\hline Straight (defined using radius of curvature) & $>1000 \mathrm{~m}$ \\
\hline
\end{tabular}

Appendix A, Methods for Determining Road Dimensions and Geometry, contains the recommended method to verify the road geometry.

\subsubsection{Available Maneuver Room}

In this test, the warning system recognizes the road edge but cannot determine if there is additional AMR. Therefore, the system should assume a default minimum AMR.

\begin{tabular}{|l|l|}
\hline Available Maneuver Room & Comment \\
\hline $15 \mathrm{~cm}(6 \mathrm{in})$ & Default minimum AMR \\
\hline
\end{tabular}

\subsubsection{Speed}

The vehicle speeds during the tests cover warning system operating range.

\begin{tabular}{|l|l|l|}
\hline Low Speed & Medium Speed & High Speed \\
\hline $64 \mathrm{~km} / \mathrm{h}(40 \mathrm{mph})$ & $89 \mathrm{~km} / \mathrm{h}(55 \mathrm{mph})$ & $113 \mathrm{~km} / \mathrm{h}(70 \mathrm{mph})$ \\
\hline
\end{tabular}

\subsubsection{Departure Rate}

The vehicle departure rates are either high or low. These rates are comparable to the rates suggested in the ROR report and in the ISO standard for testing lateral drift warning systems.

\begin{tabular}{|l|l|}
\hline Low Departure Rate & High Departure Rate \\
\hline$\leq 0.75 \mathrm{~m} / \mathrm{s}$ & $>0.75 \mathrm{~m} / \mathrm{s}$ \\
\hline
\end{tabular}

\subsubsection{Measurements}

Measurements collected during the test support the following:

1. Determine whether a warning was given 
2. Determine the desired location of a warning given the conditions

3. Determine the amount of time the warning gives driver

\subsubsection{Time of Warning}

See section 2.3.9.

\subsubsection{Longitudinal Speed}

See section 2.2.2.

\subsubsection{Distance to Road Edge}

See section 2.2.7.

\subsubsection{Lateral Velocity}

See section 2.2.4.

\subsubsection{Test Procedure}

The general procedure for the test repeats a series of road departure trajectories depicted in Figure 19.

\subsubsection{Pre-Test Procedures}

The procedures before the test are:

1. Ensure road geometry meets the test condition. Measure the geometry if it is unknown (see Appendix A, Methods for Determining Road Dimensions and Geometry).

2. Ensure lane marker characteristics meet the test conditions (see sections 2.1.12.1.12).

3. Check speedometer accuracy (see section 2.2.2).

4. Verify IMS camera calibration according to recommendations in [5]. Repeat camera calibration if camera has moved.

5. Ensure microphone detects warning system alerts and driver speech.

6. Ensure recording system is working properly.

7. Ensure GPS has sufficient satellite signals.

8. Verify road boundary for test (i.e., outer lane marker plus AMR) and practice vehicle trajectories (speeds, departure rate, etc.) until accomplished safely and reliably.

\subsubsection{Test Procedures}

The procedures during the test are:

1. Achieve speed. The driver brings the vehicle to the prescribed speed and sets the cruise control at the speed.

2. Set turn signal. The driver runs the first departure with turn signal active. Since this departure will be a True Negative, the operator documents the departure time. The operator runs the remaining departures without activating the turn signal. 
3. Execute departure. The driver steers toward the road boundary at the prescribed departure rate (high or low) until the road boundary is crossed. See section 2.2.8 for a method to achieve the prescribed departure rate.

4. Record departure rate. The assistant records the estimated departure rate as either low or high.

5. Go to start of next departure trajectory. The driver steers the vehicle back onto the road for the next departure.

6. The driver repeats the departure trajectories to fulfill the following conditions:

a. Five left departures at the low departure rate

b. Five right departures at the low departure rate

c. Five left departures at the high departure rate

d. Five right departures at the high departure rate

e. One right departure at the high departure rate with the right turn signal on.

f. One left departure at the high departure rate with the left turn signal on.

7. Repeat steps $1-6$ for the middle prescribed speed.

8. Repeat steps $1-6$ for the high-prescribed speed.

\subsubsection{Post-Test Procedures}

The procedures after the test consist of the following steps:

1. Verify IMS camera orientation has not changed. Repeat tests if changed.

\subsubsection{Evaluation Methods}

Use the following methods to evaluate warning system performance.

\subsubsection{Rate System Response}

Examine the video and audio data and rate each warning scenario.

For the road departure events:

The rating is a true positive if the system responds with a warning before the road boundary is crossed.

The rating is a false negative if the system does not respond with a warning before the road boundary is crossed.

For the cross dashed-line events:

The rating is a true positive if the system responds with a warning before the road boundary is crossed and the turn signal is inactive.

The rating is a false positive if the system responds with a warning before the road boundary is crossed and the turn signal is active. 


\subsubsection{Desired Location of Warning}

For each test run, the evaluator determines the desired location for the warning. The desired location depends on the measured departure rate and the forward speed.

1. Locate the time of warning in the video data (e.g., locate first video frame alert).

2. Measure lateral position and lateral velocity to the road edge at time of warning.

3. Use equation (1) in section 5.1.1 to determine the desired location for a warning on a straight road.

\subsubsection{Timeliness of Warning}

For each test run, the evaluator determines whether the warning is early, late or on time using steps described in section 5.1.1. The results of the timeliness evaluations are accumulated according to section 5.3.

\subsubsection{Reporting}

The evaluator compiles the test reports in accordance with the following tables.

\begin{tabular}{|l|l|}
\hline Name of Test & $\begin{array}{l}\text { Departure on One-Way Straight Road } \\
\text { with Fixed Available Maneuver Room }\end{array}$ \\
\hline Date of Test & \\
\hline Warning System ID & Name and Version Number \\
\hline
\end{tabular}


Test Run Results

\begin{tabular}{|l|l|l|l|c|c|c|c|c|c|c|c|}
\hline \multicolumn{9}{|c|}{ Factors } & \multicolumn{6}{|c|}{ Response } \\
\hline $\begin{array}{c}\text { Speed } \\
\text { mph }\end{array}$ & $\begin{array}{c}\text { Departure } \\
\text { Rate m/s }\end{array}$ & Direction & Signal & $\begin{array}{c}\text { \# of } \\
\text { runs }\end{array}$ & $\begin{array}{c}\text { Run } \\
\text { \# }\end{array}$ & $\begin{array}{c}\text { CR } \\
\text { T/F }\end{array}$ & $\begin{array}{c}\mathbf{v}_{\mathbf{f}} \\
\mathbf{m p h}\end{array}$ & $\begin{array}{c}\mathbf{v}_{\mathbf{l}} \\
\mathbf{m} / \mathbf{s}\end{array}$ & $\begin{array}{c}\mathbf{y}_{\mathbf{m}} \\
\mathbf{m}\end{array}$ & $\begin{array}{c}\mathbf{y}_{\mathbf{w}} \\
\mathbf{m}\end{array}$ & $\begin{array}{c}\mathbf{T} \\
\text { OT/E/L }\end{array}$ \\
\hline 40 & $\leq 0.75$ & Left & No & 5 & 1 & & & & & & \\
\hline$"$ & $"$ & & $n$ & & $2 \ldots$ & & & & & & \\
\hline 40 & $\leq 0.75$ & Right & No & 5 & & & & & & & \\
\hline 40 & $>0.75$ & Left & No & 5 & & & & & & & \\
\hline 40 & $>0.75$ & Right & No & 5 & & & & & & & \\
\hline 40 & $>0.75$ & Left & On & 1 & & & & & & & \\
\hline 55 & $\leq 0.75$ & Left & No & 5 & & & & & & & \\
\hline 55 & $\leq 0.75$ & Right & No & 5 & & & & & & & \\
\hline 55 & $>0.75$ & Left & No & 5 & & & & & & & \\
\hline 55 & $>0.75$ & Right & No & 5 & & & & & & & \\
\hline 55 & $>0.75$ & Left & On & 1 & & & & & & & \\
\hline 55 & $>0.75$ & Right & On & 1 & & & & & & & \\
\hline 70 & $\leq 0.75$ & Left & No & 5 & & & & & & & \\
\hline 70 & $\leq 0.75$ & Right & No & 5 & & & & & & & \\
\hline 70 & $>0.75$ & Left & No & 5 & & & & & & & \\
\hline 70 & $>0.75$ & Right & No & 5 & & & & & & & \\
\hline 70 & $>0.75$ & Left & On & 1 & & & & & & & \\
\hline 70 & $>0.75$ & Right & On & 1 & & & & & & & \\
\hline
\end{tabular}

$\mathrm{CR}=$ System gave correct response $(\mathrm{T})$ or failed to give correct response $(\mathrm{F})$. Refer to subsection 1.1 above to determine correct response.

$\mathrm{v}_{\mathrm{f}}=$ measured vehicle forward speed

$\mathrm{v}_{\mathrm{l}}=$ measured vehicle lateral speed (departure rate)

$\mathrm{y}_{\mathrm{m}}=$ measured lateral distance between the vehicle front tire and the road boundary at the time of warning

$\mathrm{y}_{\mathrm{w}}=$ the desired lateral distance at the time of warning using equation (1)

$\mathrm{T}=$ Timeliness of warning OnTime, Early or Late

Test Run Summary Results (see section 5.3)

\begin{tabular}{|c|c|c|c|c|c|c|c|c|}
\hline $\begin{array}{c}\text { Combined Results for } \\
\text { all runs }\end{array}$ & $\begin{array}{c}\# \text { of } \\
\text { runs }\end{array}$ & $\begin{array}{c}\# \\
\text { CR }\end{array}$ & $\begin{array}{c}\# \\
\text { OT }\end{array}$ & $\begin{array}{l}\# \\
\text { E }\end{array}$ & $\begin{array}{l}\# \\
\text { L }\end{array}$ & $\begin{array}{c}\text { O } \\
\text { OT }\end{array}$ & $\begin{array}{c}\text { \% } \\
\text { E }\end{array}$ & $\begin{array}{c}\text { L } \\
\text { L }\end{array}$ \\
\hline Date/time of test runs & & & & & & & & \\
\hline
\end{tabular}

False Alarm Incident Report

\begin{tabular}{|c|l|}
\hline $\begin{array}{c}\text { Run } \\
\#\end{array}$ & Probable Cause \\
\hline 1 & Example: shadows on road misinterpreted as road edge \\
\hline$\ldots$ & $\ldots$ \\
\hline
\end{tabular}

Lane Change with Turn Signal Event Evaluation

\begin{tabular}{|c|l|l|}
\hline $\begin{array}{l}\text { Number of Lane } \\
\text { Change Tests }\end{array}$ & $\begin{array}{l}\text { Number of Incorrect } \\
\text { Responses }\end{array}$ & $\begin{array}{l}\text { Number of Correct } \\
\text { Responses }\end{array}$ \\
\hline$\#$ & \multicolumn{1}{|c|}{$\#$} \\
\hline
\end{tabular}




\subsection{Two-Way Straight Road (Default minimum AMR)}

The Two-Way Straight Road (with default minimum AMR) test assesses the performance of the lateral drift warning function for road departures on undivided straight roads. This scenario falls under the "going straight on undivided non-freeway with two lanes at nonjunction" scenario identified in the NHTSA test scenario report as one of the major settings for road departure crashes [3].

The figure below illustrates the test. The road boundary depends on the type of marker the vehicle first encounters during the departure trajectory. A solid marker prohibits passing. The road boundary is the left edge of the solid marker and the available maneuver room is zero. A dashed marker allows passing and indicates a wider lane but no AMR. The warning system issues a departure alert only if the turn signal is not active. This test has no available maneuver room because the adjacent lane may have undetected on-coming traffic. The double solid lane case is similar to the solid/dashed marker in figure A.

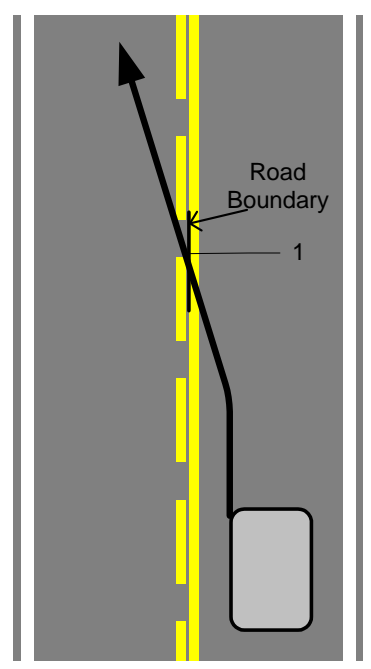

A

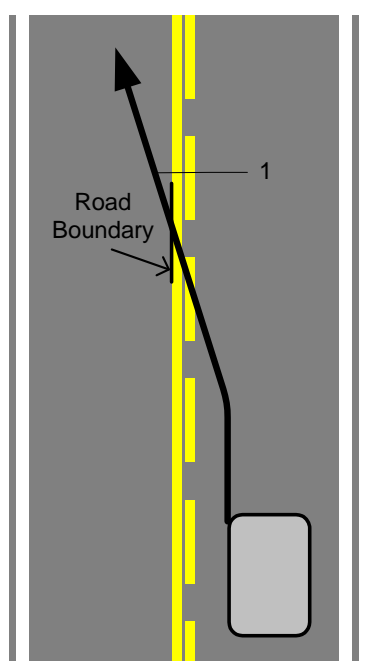

B

Figure 20. Vehicle trajectory during test

\subsubsection{Events and System Response}

The following table defines the events for this test and the correct warning signal the system shall provide. See the figure above to locate the event (e.g., Id A1 is label 1 in figure A). 


\begin{tabular}{|c|l|l|}
\hline Id & \multicolumn{1}{|c|}{ Event } & \multicolumn{1}{c|}{ Correct Response } \\
\hline A1 & $\begin{array}{l}\text { Road Departure with } \\
\text { passing not permitted }\end{array}$ & 1. Issue road departure warning \\
\hline B1 & $\begin{array}{l}\text { Road Departure with } \\
\text { passing permitted }\end{array}$ & $\begin{array}{l}\text { 1. Issue no warning if the correct turn signal active. } \\
\text { 2. Issue a road departure warning if no turn signal } \\
\text { active or incorrect turn signal active. }\end{array}$ \\
\hline
\end{tabular}

\subsubsection{Test Conditions}

The conditions for this test include the following.

\subsubsection{Road Geometry}

The road geometry for the test is the following:

\begin{tabular}{|l|l|}
\hline Road Geometry & Dimension \\
\hline Straight (defined using radius of curvature) & $>1000 \mathrm{~m}$ \\
\hline
\end{tabular}

Appendix A, Methods for Determining Road Dimensions and Geometry, contains the recommended method to verify the geometry.

\subsubsection{Available Maneuver Room}

In this test, the warning system recognizes the road edge but cannot determine if there is on-coming traffic. Therefore, the AMR equals zero.

\begin{tabular}{|l|l|}
\hline Available Maneuver Room & Comment \\
\hline $0 \mathrm{~cm}$ & Not allowed to cross over the lane marker \\
\hline
\end{tabular}

\subsubsection{Speed}

The vehicle speeds during the tests cover the warning system operating range.

\begin{tabular}{|l|l|l|}
\hline Low Speed & Medium Speed & High Speed \\
\hline $64 \mathrm{~km} / \mathrm{h}(40 \mathrm{mph})$ & $89 \mathrm{~km} / \mathrm{h}(55 \mathrm{mph})$ & $113 \mathrm{~km} / \mathrm{h}(70 \mathrm{mph})$ \\
\hline
\end{tabular}

\subsubsection{Departure Rate}

The vehicle departure rates during the tests fall within two ranges. These rates are comparable to the rates suggested in the ROR report and in the ISO standard for testing lateral drift warning systems.

\begin{tabular}{|l|l|}
\hline Low Departure Rate & High Departure Rate \\
\hline$\leq 0.75 \mathrm{~m} / \mathrm{s}$ & $>0.75 \mathrm{~m} / \mathrm{s}$ \\
\hline
\end{tabular}

\subsubsection{Measurements}

Measurements collected during the test support the following:

1. Determine whether a warning was given

2. Determine the desired location of a warning given the conditions 
3. Determine the amount of time the warning gives driver

\subsubsection{Time of Warning}

See section 2.3.9.

\subsubsection{Longitudinal Speed}

See section 2.2.2.

\subsubsection{Distance to Road Edge}

See section 2.2.7.

\subsubsection{Lateral Velocity}

See section 2.2.4.

\subsubsection{Test Procedure}

The general procedure for the test is to repeat a series of road departure trajectories depicted in Figure 20.

\subsubsection{Pre-Test Procedures}

The procedures before the test are:

1. Ensure road geometry meets the test condition. Measure the geometry if it is unknown (see Appendix A, Methods for Determining Road Dimensions and Geometry).

2. Ensure lane marker characteristics meet the test conditions (see sections 2.1.12.1.12).

3. Check speedometer accuracy (see section 2.2.2).

4. Verify IMS camera calibration according to recommendations in [5]. Repeat camera calibration if camera has moved.

5. Ensure microphone detects warning system alerts and driver speech.

6. Ensure recording system is working properly.

7. Ensure GPS has sufficient satellite signals.

8. Verify road boundary for test (i.e., outer lane marker plus AMR) and practice vehicle trajectories (speeds, departure rate, etc.) until accomplished safely and reliably.

\subsubsection{Test Procedures}

The procedures during the test are:

1. Achieve speed. The driver brings the vehicle to the prescribed speed and sets the cruise control at the speed.

2. Set turn signal. The driver runs one departure with turn signal active and documents the departure time. The driver runs the remaining departures with the turn signal inactive. 
3. Execute departure. The driver steers toward the road boundary at the prescribed departure rate (high or low) until the road boundary is crossed. See section 2.2.8 for a method to achieve the prescribed departure rate.

4. Record departure rate. The assistant records the estimated departure rate as either low or high.

5. Go to start of next departure trajectory. The driver steers the vehicle back onto the road for the next departure

6. Repeat steps 1-5 for:

a. Five left departures in no passing zone at the low departure rate

b. Five left departures in no passing zone at the high departure rate

c. Five left departures in passing zone at the low departure rate

d. Five left departures in passing zone at the high departure rate

e. One left departure in passing zone at the high departure rate with turn signal on.

7. Repeat steps $1-6$ for the middle prescribed speed.

8. Repeat steps $1-6$ for the high prescribed speed.

\subsubsection{Post-Test Procedures}

The procedures after the test are:

1. Verify IMS camera orientation has not changed. Repeat tests if changed.

\subsubsection{Evaluation Methods}

Use the following methods to evaluate warning system performance.

\subsubsection{Rate System Response}

Examine the video and audio data and rate each warning scenario.

For the no passing permitted road departure event:

The rating is a true positive if the system responds with a warning before the road boundary is crossed.

The rating is a false negative if the system does not respond with a warning before the road boundary is crossed.

For the passing permitted road departure event:

The rating is a true positive if the system responds with a warning before the road boundary is crossed and the turn signal is inactive.

The rating is a false positive if the system responds with a warning before the road boundary is crossed and the turn signal is active. 


\subsubsection{Desired Location of Warning}

For each test run, the evaluator determines the desired location of the warning. The desired warning location varies with the departure rate and the forward speed.

1. Locate the time of warning in the video data (e.g., the first video frame with the alert).

2. Measure lateral position and lateral velocity to the road edge at the time of the warning.

3. With equation (1) in section 5.1.1, determine the desired location for a warning on a straight road.

\subsubsection{Timeliness of Warning}

For each test run, the evaluator determines whether the warning is early, late or on time using steps described in section 5.1.1. The results of the timeliness evaluations are accumulated according to section 5.3.

\subsubsection{Reporting}

The evaluator completes the following tables with information from each test.

\begin{tabular}{|l|l|}
\hline Name of Test & $\begin{array}{l}\text { Departure on Un-Divided (Two-Way) } \\
\text { Straight Road with No AMR }\end{array}$ \\
\hline Date of Test & \\
\hline Warning System ID & Name and Version Number \\
\hline
\end{tabular}

Test Run Results

\begin{tabular}{|c|c|c|c|c|c|c|c|c|c|c|c|c|}
\hline \multicolumn{6}{|c|}{ Factors } & \multicolumn{7}{|c|}{ Response } \\
\hline $\begin{array}{l}\text { Speed } \\
\text { mph }\end{array}$ & $\begin{array}{c}\text { Departure } \\
\text { Rate m/s }\end{array}$ & Direction & $\begin{array}{l}\text { Pass } \\
\text { Zone }\end{array}$ & Signal & $\begin{array}{l}\text { \# of } \\
\text { runs }\end{array}$ & $\stackrel{\text { Run }}{\#}$ & $\begin{array}{l}\text { CR } \\
\text { T/F }\end{array}$ & $\begin{array}{c}\mathbf{v}_{f} \\
\text { mph }\end{array}$ & $\begin{array}{l}\mathbf{v}_{\mathbf{l}} \\
\mathrm{m} / \mathrm{s}\end{array}$ & $\begin{array}{c}\mathbf{y}_{\mathbf{m}} \\
\mathbf{m}\end{array}$ & $\begin{array}{c}\mathbf{y}_{\mathbf{w}} \\
\mathbf{m}\end{array}$ & $\begin{array}{c}\text { T } \\
\text { OT/E/L }\end{array}$ \\
\hline 40 & $\leq 0.75$ & Left & NP & None & 5 & 1 & & & & & & \\
\hline$"$ & $"$ & $"$ & $"$ & $"$ & $"$ & $2 \ldots$ & & & & & & \\
\hline 40 & $>0.75$ & Left & NP & None & 5 & & & & & & & \\
\hline 40 & $\leq 0.75$ & Left & $\mathrm{P}$ & None & 5 & & & & & & & \\
\hline 40 & $>0.75$ & Left & $\mathrm{P}$ & None & 5 & & & & & & & \\
\hline 40 & $>0.75$ & Left & $\mathrm{P}$ & $\mathrm{ON}$ & 1 & & & & & & & \\
\hline 55 & $\leq 0.75$ & Left & NP & None & 5 & & & & & & & \\
\hline 55 & $>0.75$ & Left & NP & None & 5 & & & & & & & \\
\hline 55 & $\leq 0.75$ & Left & $\mathrm{P}$ & None & 5 & & & & & & & \\
\hline 55 & $>0.75$ & Left & $\mathrm{P}$ & None & 5 & & & & & & & \\
\hline 55 & $>0.75$ & Left & $\mathrm{P}$ & $\mathrm{ON}$ & 1 & & & & & & & \\
\hline 70 & $\leq 0.75$ & Left & NP & None & 5 & & & & & & & \\
\hline 70 & $>0.75$ & Left & NP & None & 5 & & & & & & & \\
\hline 70 & $\leq 0.75$ & Left & $\mathrm{P}$ & None & 5 & & & & & & & \\
\hline 70 & $>0.75$ & Left & $\mathrm{P}$ & None & 5 & & & & & & & \\
\hline 70 & $>0.75$ & Left & $\mathrm{P}$ & $\mathrm{ON}$ & 1 & & & & & & & \\
\hline
\end{tabular}

$\mathrm{CR}=$ System gave correct response $(\mathrm{T})$ or failed to give correct response $(\mathrm{F})$. Refer to subsection 1.1 above to determine correct response. 


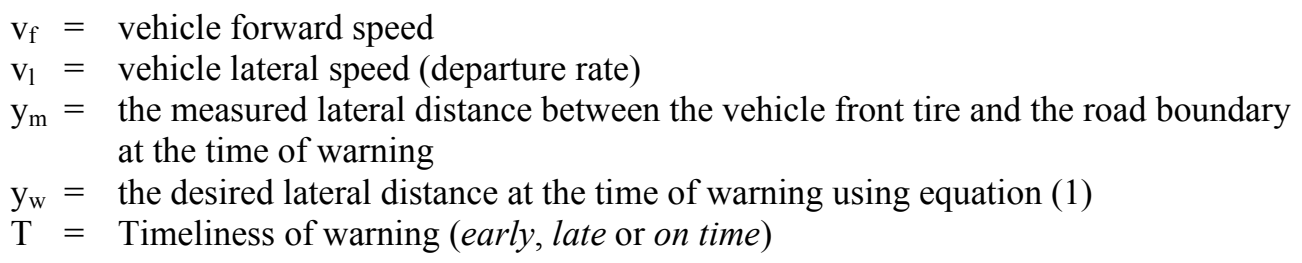

Test Run Summary Results (see section 5.3)

\begin{tabular}{|c|c|c|c|c|c|c|c|c|}
\hline $\begin{array}{c}\text { Combined Results for } \\
\text { all runs }\end{array}$ & $\begin{array}{l}\text { \# of } \\
\text { runs }\end{array}$ & $\begin{array}{c}\# \\
\text { CR }\end{array}$ & $\begin{array}{c}\# \\
\text { OT }\end{array}$ & $\begin{array}{l}\# \\
\mathbf{E}\end{array}$ & $\begin{array}{l}\# \\
\text { L }\end{array}$ & $\begin{array}{l}\% \\
\text { OT }\end{array}$ & $\begin{array}{l}\% \\
\text { E }\end{array}$ & $\begin{array}{l}\text { \% } \\
\text { L }\end{array}$ \\
\hline Date/time of test runs & & & & & & & & \\
\hline
\end{tabular}

False Alarm Incident Report

\begin{tabular}{|c|l|}
\hline $\begin{array}{c}\text { Run } \\
\#\end{array}$ & Probable Cause \\
\hline 1 & Example: shadows on road misinterpreted as road edge \\
\hline$\ldots$ & $\ldots$ \\
\hline
\end{tabular}

Lane Change with Turn Signal Event Evaluation

\begin{tabular}{|c|l|l|}
\hline $\begin{array}{l}\text { Number of Lane } \\
\text { Change Tests }\end{array}$ & $\begin{array}{l}\text { Number of Incorrect } \\
\text { Responses }\end{array}$ & $\begin{array}{l}\text { Number of Correct } \\
\text { Responses }\end{array}$ \\
\hline$\#$ & \multicolumn{1}{|c|}{$\#$} & $\#$ \\
\hline
\end{tabular}

\subsection{Curved Road (Default minimum AMR)}

The Curved Road (default minimum AMR) test examines the performance of the lateral drift warning function for road departures on a curved road. This scenario is not part of the curve speed warning function. During the Curved Road test, the vehicle travels within the established speed limit for the roadway. The test uses three curves of different radii: a slow speed curve, a medium speed curve and a high-speed curve.

In this scenario, the vehicle travels straight until a road departure occurs (Figure 21). The driver uses a guide target to maintain the straight road trajectory during the departure. The test area must have sufficient room for safe recovery after the road departure.

Run the tests in the opposite direction to test a left-side departure (e.g. for a divided highway). 


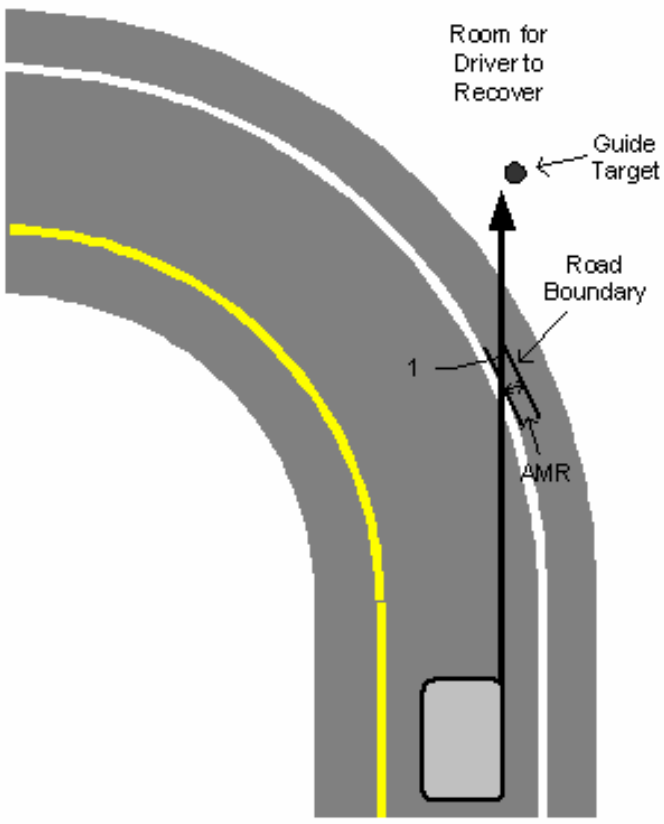

Figure 21. Vehicle trajectory during test

\subsubsection{Events and System Response}

The following table defines the event for this test and the correct warning signal response. The numeral in Figure 21 above displays the proper trajectory for the event.

\begin{tabular}{|c|l|l|}
\hline Id & \multicolumn{1}{|c|}{ Event } & \multicolumn{1}{c|}{ Correct Response } \\
\hline 1 & Crossed solid line & Issue crossed solid line warning \\
\hline
\end{tabular}

\subsubsection{Test Conditions}

The conditions for this test include the following.

\subsubsection{Road Geometry}

The curve radius for this test should be appropriate for the low speed, medium speed and high-speed conditions. The following equation calculates the curve radius for each speed condition using lateral acceleration limits $\left(a_{\text {lat }}\right)$ from section 2.3.5.

$$
r=\frac{v^{2}}{a_{\text {lat }}}
$$

To simplify setting up the test, a curve radius may fall within a range of radii by using the lateral acceleration limits for sensitivities 2 and 4.

\begin{tabular}{|l|l|}
\hline Road Geometry & Dimension \\
\hline Low speed curve, $64 \mathrm{~km} / \mathrm{h}(40 \mathrm{mph})$ & $91 \mathrm{~m}-136 \mathrm{~m}$ \\
\hline
\end{tabular}


Medium speed curve, $89 \mathrm{~km} / \mathrm{h}$ (55 mph)

$172 \mathrm{~m}-257 \mathrm{~m}$

Low speed curve, $113 \mathrm{~km} / \mathrm{h}(70 \mathrm{mph})$

$278 \mathrm{~m}-417 \mathrm{~m}$

Appendix A, Methods for Determining Road Dimensions and Geometry, contains the recommended method to verify the geometry.

\subsubsection{Available Maneuver Room}

In this test, the warning system recognizes the road edge but cannot determine if there is additional AMR. Therefore, the system should assume a default minimum AMR.

\begin{tabular}{|l|l|}
\hline Available Maneuver Room & Comment \\
\hline $15 \mathrm{~cm}(6 \mathrm{in})$ & Default minimum AMR \\
\hline
\end{tabular}

\subsubsection{Speed}

The vehicle speeds during the tests cover the warning system operating range. The operator uses appropriate curve radii as the trajectory speed changes.

\begin{tabular}{|l|l|l|}
\hline Low Speed & Medium Speed & High Speed \\
\hline $64 \mathrm{~km} / \mathrm{h}(40 \mathrm{mph})$ & $89 \mathrm{~km} / \mathrm{h}(55 \mathrm{mph})$ & $113 \mathrm{~km} / \mathrm{h}(70 \mathrm{mph})$ \\
\hline
\end{tabular}

\subsubsection{Measurements}

Measurements collected during the test support the following:

1. Determine whether a warning was given

2. Determine the desired location of a warning given the conditions

3. Determine the amount of time the warning gives driver

\subsubsection{Time of Warning}

See section 2.3.9.

\subsubsection{Longitudinal Speed}

See section 2.2.2.

\subsubsection{Distance to Road Edge}

See section 2.2.7.

\subsubsection{Distance to Curve Entry Point}

See section 2.2.10.

\subsubsection{Test Procedure}

In this test, the driver repeats a series of road departure trajectories as depicted in Figure 21 above.

\subsubsection{Pre-Test Procedures}

The procedures before the test are: 
1. Ensure road geometry meets the test condition. Measure the geometry if it is unknown (see Appendix A, Methods for Determining Road Dimensions and Geometry).

2. Ensure lane marker characteristics meet the test conditions (see sections 2.1.12.1.12).

3. Check speedometer accuracy (see section 2.2.2).

4. Verify IMS camera calibration according to recommendations in [5]. Repeat camera calibration if camera has moved.

5. Ensure microphone detects warning system alerts and driver speech.

6. Ensure recording system is working properly.

7. Ensure GPS has sufficient satellite signals.

8. Verify road boundary for test (i.e., outer lane marker plus AMR) and practice vehicle trajectories (speeds, departure rate, etc.) until accomplished safely and reliably.

\subsubsection{Test Procedures}

The procedures during the test are:

1. Achieve speed. The driver brings the vehicle to the prescribed speed (low speed curve is $64 \mathrm{~km} / \mathrm{h}(40 \mathrm{mph}))$. The driver uses the cruise control if available.

2. Straighten vehicle before curve entry. Before entering the curve, the driver centers the vehicle in the lane using the lane markers as a visual guide. The vehicle remains centered into the curve.

3. Maintain straight trajectory into the curve. As the vehicle enters the curve, the driver uses a guide target to maintain a straight trajectory through the departure.

4. Depart road. The driver crosses over the lane marker and then recovers from the departure.

5. Repeat the departure trajectories for:
a. Five right departures

b. Five left departures

6. Repeat steps $1-5$ for the middle speed curve.

7. Repeat steps $1-5$ for the high-speed curve.

\subsubsection{Post-Test Procedures}

The procedures after the test are:

1. Verify IMS camera orientation has not changed. Repeat tests if changed.

\subsubsection{Evaluation Methods}

Use the following methods to evaluate warning system performance.

\subsubsection{Rate System Response}

Examine the video and audio data and rate each warning scenario. 
The rating is a true positive if the system responds with a warning before the road boundary is crossed.

The rating is a false negative if the system does not respond with a warning before the road boundary is crossed.

\subsubsection{Desired Location of Warning}

For each test run, the evaluator determines the desired location of the warning. This will vary depending on the measured departure rate and forward speed.

1. Locate the time of warning in the video data (for example, by locating video frame where alert is first displayed).

2. Measure lateral position to the road edge at time of warning.

3. Use equation (4) in section 5.1.2 to determine the desired location for a warning on a curve.

\subsubsection{Timeliness of Warning}

For each test run, the evaluator determines whether the warning is early, late or on time using steps described in section Error! Reference source not found.. The results of the timeliness evaluations are accumulated according to section 5.3.

\subsubsection{Reporting}

The evaluator completes the following tables from the data from each test.

\begin{tabular}{|l|l|}
\hline Name of Test & $\begin{array}{l}\text { Departure on Divided Curved Road with Fixed Available } \\
\text { Maneuver Room }\end{array}$ \\
\hline Date of Test & \\
\hline Warning System ID & Name and Version Number \\
\hline
\end{tabular}

Test Run Results

\begin{tabular}{|l|l|l|l|l|l|c|c|c|c|}
\hline \multicolumn{3}{|c|}{ Factors } & \multicolumn{6}{|c|}{ Results } \\
\hline Curve & $\begin{array}{c}\text { Speed } \\
\text { mph }\end{array}$ & Direction & $\begin{array}{c}\text { \# of } \\
\text { runs }\end{array}$ & $\begin{array}{c}\text { Run } \\
\#\end{array}$ & $\begin{array}{c}\text { CR } \\
\text { T/F }\end{array}$ & $\begin{array}{c}\mathbf{v}_{\mathbf{f}} \\
\mathbf{m p h}\end{array}$ & $\begin{array}{c}\mathbf{y}_{\mathbf{m}} \\
\mathbf{m}\end{array}$ & $\begin{array}{c}\mathbf{x}_{\mathbf{w}} \\
\mathbf{m}\end{array}$ & $\begin{array}{c}\mathbf{T} \\
\text { OT/E/L }\end{array}$ \\
\hline LS & 40 & Left & 5 & 1 & & & & & \\
\hline$"$ & $"$ & $"$ & & $2 \ldots$ & & & & & \\
\hline LS & 40 & Right & 5 & & & & & & \\
\hline MS & 55 & Left & 5 & & & & & & \\
\hline MS & 55 & Right & 5 & & & & & & \\
\hline HS & 70 & Left & 5 & & & & & & \\
\hline HS & 70 & Right & 5 & & & & & & \\
\hline
\end{tabular}

Curve $=$ Low speed curve, medium speed curve or high speed curve

$\mathrm{CR}=$ System gave correct response $(\mathrm{T})$ or failed to give correct response $(\mathrm{F})$. Refer to subsection 1.1 above to determine correct response.

$\mathrm{v}_{\mathrm{f}}=$ vehicle forward speed 
$\mathrm{y}_{\mathrm{m}}=$ the measured lateral distance between the vehicle front tire and the road boundary at the time of warning

$\mathrm{x}_{\mathrm{W}}=$ the desired distance to curve entry point at the time of warning using equation (4)

$\mathrm{T}=$ Timeliness of warning (early, late or on time)

Test Run Summary Results (see section 5.3)

\begin{tabular}{|c|c|c|c|c|c|c|c|c|}
\hline $\begin{array}{c}\text { Combined Results for } \\
\text { all runs }\end{array}$ & $\begin{array}{c}\# \text { of } \\
\text { runs }\end{array}$ & $\begin{array}{c}\# \\
\text { CR }\end{array}$ & $\begin{array}{c}\# \\
\text { OT }\end{array}$ & $\begin{array}{c}\# \\
\text { E }\end{array}$ & $\begin{array}{c}\# \\
\text { L }\end{array}$ & $\begin{array}{c}\text { \% } \\
\text { OT }\end{array}$ & $\begin{array}{c}\text { \% } \\
\text { E }\end{array}$ & $\begin{array}{c}\text { L } \\
\text { L }\end{array}$ \\
\hline Date/time of test runs & & & & & & & & \\
\hline
\end{tabular}

False Alarm Incident Report

\begin{tabular}{|c|l|}
\hline $\begin{array}{c}\text { Run } \\
\#\end{array}$ & Probable Cause \\
\hline 1 & Example: shadows on road misinterpreted as road edge \\
\hline$\ldots$ & $\ldots$ \\
\hline
\end{tabular}




\section{Tests for Departures on Roads with Maximum AMR}

The Departure on Roads with Maximum AMR tests evaluate warning system response to road departures where a shoulder provides increased available maneuver room. These tests do not assess the ability of the system to detect obstacles on the shoulder.

Therefore, these tests do not provide a complete assessment of a fully functional warning system. A warning system must also pass the "Tests for Departures Toward an Obstacle" that appear in section 8.

These tests assess the capability of a warning system to detect the shoulder and measure its width. The transition between on-road and off-road must have some discriminating visual property, such as color or texture. The tests employ two off-road materials: dirt and artificial grass. The off-road material lies upon a paved surface so that the vehicle may depart from the road with minimal risk.

The tests employ two shoulder widths. The large shoulder width is twice the maximum AMR. The system should detect the large shoulder but limit the AMR to the maximum in order to provide consistent warnings. The small shoulder is half the maximum AMR. The system should detect the small shoulder and issue the warning earlier than for a large shoulder. An earlier warning indicates the system successfully detected the decreased shoulder width.

The test scenarios include departures on a straight road and on a curved road. 


\subsection{Straight Road with Shoulder (Maximum AMR)}

The Straight Road with Shoulder test assesses the warning system's ability to detect the shoulder width and to adjust the warning location on a straight road departure.

The figure below illustrates the test scenario. The left-side departure is similar.
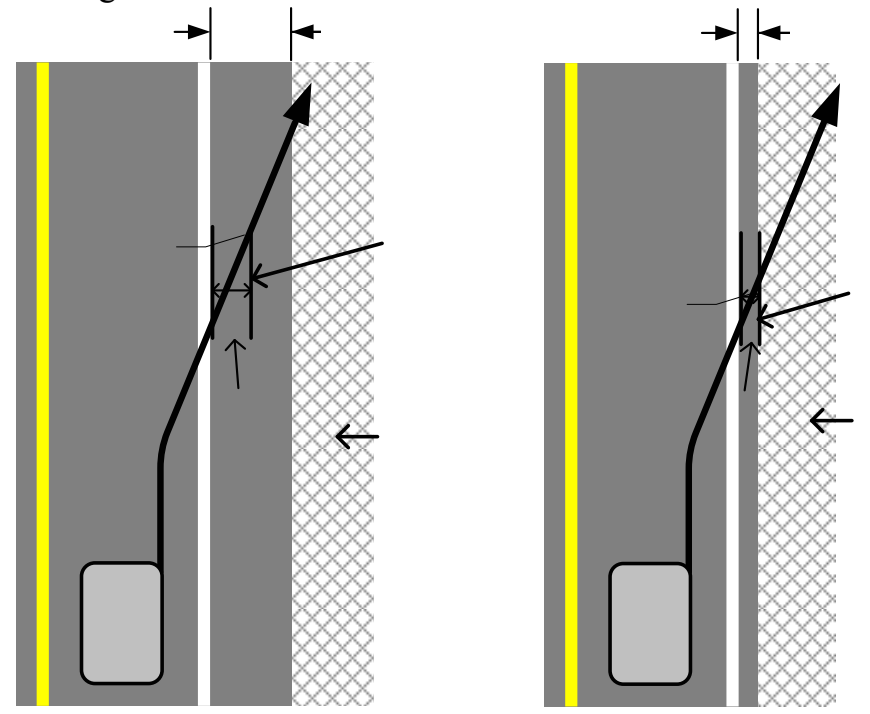

Figure 22. Straight Road with Shoulder Test Trajectory

\subsubsection{Events and System Response}

The following table defines the events for this test and the correct warning response. See the figure above to locate the event (e.g., Id A1 is label 1 in figure A).

\begin{tabular}{|c|l|l|}
\hline Id & \multicolumn{1}{|c|}{ Event } & \multicolumn{1}{c|}{ Correct Response } \\
\hline A1 & Road departure & 1. Issue road departure warning \\
\hline B1 & Same as A1 & 1. Same as A1, except earlier \\
\hline
\end{tabular}

\subsubsection{Test Conditions}

The conditions for this test include the following.

\subsubsection{Road Geometry}

The road geometry for the test is:

\begin{tabular}{|l|l|}
\hline Road Geometry & Dimension \\
\hline Straight (defined using radius of curvature) & $>1000 \mathrm{~m}$ \\
\hline
\end{tabular}


Appendix A, Methods for Determining Road Dimensions and Geometry, contains the recommended method to verify the road geometry.

\subsubsection{Available Maneuver Room}

In this test, shoulder width affects the AMR. However, the test limits the AMR to a default maximum value to prevent large variations in warning location due to large variations in shoulder width.

\begin{tabular}{|l|l|}
\hline Available Maneuver Room & Comment \\
\hline $1 \mathrm{~m}(39 \mathrm{in})$ & Default maximum AMR \\
\hline
\end{tabular}

\subsubsection{Speed}

The vehicle speeds during the tests cover the warning system operating range.

\begin{tabular}{|l|l|l|}
\hline Low Speed & Medium Speed & High Speed \\
\hline $64 \mathrm{~km} / \mathrm{h}(40 \mathrm{mph})$ & $89 \mathrm{~km} / \mathrm{h}(55 \mathrm{mph})$ & $113 \mathrm{~km} / \mathrm{h}(70 \mathrm{mph})$ \\
\hline
\end{tabular}

\subsubsection{Departure Rate}

The vehicle departure rates during the tests fall within two ranges. These rates are comparable to the rates suggested in the ROR report and in the ISO standard for testing lateral drift warning systems.

\begin{tabular}{|l|l|}
\hline Low Departure Rate & High Departure Rate \\
\hline$\leq 0.75 \mathrm{~m} / \mathrm{s}$ & $>0.75 \mathrm{~m} / \mathrm{s}$ \\
\hline
\end{tabular}

\subsubsection{Shoulder Width}

This test uses two shoulder widths. The small shoulder is one half the default maximum AMR and the large shoulder is twice the AMR. Update these values if the default minimum or maximum AMR changes.

\begin{tabular}{|l|l|}
\hline Shoulder Width Name & \multicolumn{1}{c|}{ Dimension } \\
\hline Small Shoulder & $0.5 \mathrm{~m}(19.5 \mathrm{in})$ \\
\hline Large Shoulder & $2 \mathrm{~m}$ \\
\hline
\end{tabular}

\subsubsection{Off-Road Material}

These tests use two types of material to simulate off-road surfaces. The operator places the material on a wide shoulder to reduce the visual width of the shoulder to the widths specified above.

\begin{tabular}{|l|l|}
\hline \multicolumn{1}{|c|}{ Off-road } & \multicolumn{1}{c|}{ Characteristics } \\
\hline Dirt & Brown or variations. \\
\hline Grass & Green artificial grass material \\
\hline
\end{tabular}




\subsubsection{Measurements}

Measurements collected during the test support the following:

1. Determine whether a warning was given

2. Determine the desired location of a warning given the conditions

3. Determine the amount of time the warning gives driver

\subsubsection{Time of Warning}

See section 2.3.9.

\subsubsection{Longitudinal Speed}

See section 2.2.2.

\subsubsection{Distance to Road Edge}

See section 2.2.7.

\subsubsection{Lateral Velocity}

See section 2.2.4.

\subsubsection{Test Procedure}

The test repeats a series of road departure trajectories as depicted in Figure 22.

\subsubsection{Pre-Test Procedures}

The procedures before the test are:

1. Ensure road geometry meets the test condition. Measure the geometry if it is unknown (see Appendix A, Methods for Determining Road Dimensions and Geometry).

2. Ensure lane marker characteristics meet the test conditions (see sections 2.1.12.1.12).

3. Check speedometer accuracy (see section 2.2.2).

4. Verify IMS camera calibration according to recommendations in [5]. Repeat camera calibration if camera has moved.

5. Ensure microphone detects warning system alerts and driver speech.

6. Ensure recording system is working properly.

7. Ensure GPS has sufficient satellite signals.

8. Verify road boundary for test (i.e., outer lane marker plus AMR) and practice vehicle trajectories (speeds, departure rate, etc.) until accomplished safely and reliably.

9. Set AMR width with dirt off-road material.

\subsubsection{Test Procedures}

The procedures during the test are: 
1. Achieve speed. The driver brings the vehicle to the prescribed speed. The driver may use cruise control if the vehicle is properly equipped.

2. Execute departure. The driver steers toward the road boundary at the prescribed departure rate (high or low) until the road boundary (dirt or grass) is crossed. See section 2.2.8 for a method to achieve the prescribed departure rate.

3. Record departure rate. The assistant records the estimated departure rate as either low or high.

4. Go to start of next departure trajectory. The driver steers the vehicle back onto the road for the next departure.

5. Repeat steps 1-4 for:

a. Five right departures at the low departure rate

b. Five right departures at the high departure rate

6. Repeat steps $1-5$ for the prescribed middle speed.

7. Repeat steps $1-5$ for the prescribed high speed.

8. Repeat steps $1-7$ with the small shoulder width.

9. Repeat steps $1-8$ for grass off-road material.

\subsubsection{Post-Test Procedures}

The procedures after the test are:

1. Verify IMS camera orientation has not changed. Repeat tests if changed.

\subsubsection{Evaluation Methods}

Use the following methods to evaluate warning system performance.

\subsubsection{Rate System Response}

Examine the video and audio data and rate each warning scenario.

The rating is a true positive if the system responds with a warning before the road boundary is crossed.

The rating is a false negative if the system does not respond with a warning before the road boundary is crossed.

\subsubsection{Desired Location of Warning}

For each test run, the evaluator determines the desired location of the warning. The desired location varies with the lane curvature and the forward speed.

1. Locate the time of warning in the video data (e.g., first video frame with an alert).

2. Measure lateral position and lateral velocity to the road edge at time of warning.

3. Use equation (1) in section 5.1.1 to determine the desired location for a warning on a straight road. 


\subsubsection{Timeliness of Warning}

For each test run, the evaluator determines whether the warning is early, late or on time using steps described in section 5.1.1. The results of the timeliness evaluations are accumulated according to section 5.3.

\subsubsection{Reporting}

The evaluator completes the following tables from the data in each test.

\begin{tabular}{|l|l|}
\hline Name of Test & Departure on Straight Road with Shoulder \\
\hline Date of Test & \\
\hline Warning System ID & Name and Version Number \\
\hline
\end{tabular}

Test Run Results

\begin{tabular}{|c|c|c|c|c|c|c|c|c|c|c|c|c|}
\hline \multicolumn{6}{|c|}{ Factors } & \multicolumn{7}{|c|}{ Results } \\
\hline $\begin{array}{l}\text { Offroad } \\
\text { Mat. }\end{array}$ & $\begin{array}{c}\text { Speed } \\
\text { mph }\end{array}$ & $\begin{array}{l}\text { Departure } \\
\text { Rate } \mathbf{m} / \mathrm{s}\end{array}$ & Direction & Signal & $\begin{array}{l}\text { \# of } \\
\text { runs }\end{array}$ & $\begin{array}{c}\text { Run } \\
\#\end{array}$ & $\begin{array}{l}\text { CR } \\
\text { T/F }\end{array}$ & $\begin{array}{c}\mathbf{v}_{\mathbf{f}} \\
\mathbf{m p h}\end{array}$ & $\begin{array}{l}\mathbf{v}_{\mathbf{l}} \\
\mathrm{m} / \mathrm{s}\end{array}$ & $\begin{array}{c}\mathbf{y}_{\mathbf{m}} \\
\mathbf{m}\end{array}$ & $\begin{array}{c}\mathbf{y}_{\mathbf{w}} \\
\mathbf{m}\end{array}$ & $\begin{array}{c}T \\
\text { OT/E/L } \\
\end{array}$ \\
\hline Dirt & 40 & $\leq 0.75$ & Left & No & 5 & 1 & & & & & & \\
\hline$"$ & $"$ & " & $"$ & $"$ & & $2 \ldots$ & & & & & & \\
\hline Dirt & 40 & $\leq 0.75$ & Right & No & 5 & & & & & & & \\
\hline Dirt & 40 & $>0.75$ & Left & No & 5 & & & & & & & \\
\hline Dirt & 40 & $>0.75$ & Right & No & 5 & & & & & & & \\
\hline Dirt & 40 & $>0.75$ & Left & On & 1 & & & & & & & \\
\hline Dirt & 55 & $\leq 0.75$ & Left & No & 5 & & & & & & & \\
\hline Dirt & 55 & $\leq 0.75$ & Right & No & 5 & & & & & & & \\
\hline Dirt & 55 & $>0.75$ & Left & No & 5 & & & & & & & \\
\hline Dirt & 55 & $>0.75$ & Right & No & 5 & & & & & & & \\
\hline Dirt & 55 & $>0.75$ & Left & On & 1 & & & & & & & \\
\hline Dirt & 55 & $>0.75$ & Right & On & 1 & & & & & & & \\
\hline Dirt & 70 & $\leq 0.75$ & Left & No & 5 & & & & & & & \\
\hline Dirt & 70 & $\leq 0.75$ & Right & No & 5 & & & & & & & \\
\hline Dirt & 70 & $>0.75$ & Left & No & 5 & & & & & & & \\
\hline Dirt & 70 & $>0.75$ & Right & No & 5 & & & & & & & \\
\hline Dirt & 70 & $>0.75$ & Left & On & 1 & & & & & & & \\
\hline Dirt & 70 & $>0.75$ & Right & On & 1 & & & & & & & \\
\hline Grass & 40 & $\leq 0.75$ & Left & No & 5 & & & & & & & \\
\hline Grass & 40 & $\leq 0.75$ & Right & No & 5 & & & & & & & \\
\hline Grass & 40 & $>0.75$ & Left & No & 5 & & & & & & & \\
\hline Grass & 40 & $>0.75$ & Right & No & 5 & & & & & & & \\
\hline Grass & 40 & $>0.75$ & Left & On & 1 & & & & & & & \\
\hline Grass & 55 & $\leq 0.75$ & Left & No & 5 & & & & & & & \\
\hline Grass & 55 & $\leq 0.75$ & Right & No & 5 & & & & & & & \\
\hline Grass & 55 & $>0.75$ & Left & No & 5 & & & & & & & \\
\hline Grass & 55 & $>0.75$ & Right & No & 5 & & & & & & & \\
\hline Grass & 55 & $>0.75$ & Left & On & 1 & & & & & & & \\
\hline Grass & 55 & $>0.75$ & Right & On & 1 & & & & & & & \\
\hline
\end{tabular}




\begin{tabular}{|l|l|l|l|l|l|l|l|l|l|l|l|l|}
\hline Grass & 70 & $\leq 0.75$ & Left & No & 5 & & & & & & & \\
\hline Grass & 70 & $\leq 0.75$ & Right & No & 5 & & & & & & & \\
\hline Grass & 70 & $>0.75$ & Left & No & 5 & & & & & & & \\
\hline Grass & 70 & $>0.75$ & Right & No & 5 & & & & & & & \\
\hline Grass & 70 & $>0.75$ & Left & On & 1 & & & & & & & \\
\hline Grass & 70 & $>0.75$ & Right & On & 1 & & & & & & & \\
\hline
\end{tabular}

$\mathrm{CR}=$ System gave correct response $(\mathrm{T})$ or failed to give correct response $(\mathrm{F})$. Refer to subsection 1.1 above to determine correct response.

$\mathrm{v}_{\mathrm{f}}=$ measured vehicle forward speed

$\mathrm{v}_{\mathrm{l}}=$ measured vehicle lateral speed (departure rate)

$\mathrm{y}_{\mathrm{m}}=$ measured lateral distance between the vehicle front tire and the road boundary at the time of warning

$\mathrm{y}_{\mathrm{w}}=$ the desired lateral distance at the time of warning using equation (1)

$\mathrm{T}=$ Timeliness of warning (early, late or on time)

Test Run Summary Results (see section 5.3)

\begin{tabular}{|c|c|c|c|c|c|c|c|c|}
\hline $\begin{array}{c}\text { Combined Results for } \\
\text { all runs }\end{array}$ & $\begin{array}{l}\text { \# of } \\
\text { runs }\end{array}$ & $\begin{array}{c}\# \\
\text { CR }\end{array}$ & $\begin{array}{c}\# \\
\text { OT }\end{array}$ & $\begin{array}{l}\# \\
\mathbf{E}\end{array}$ & $\begin{array}{l}\# \\
\mathbf{L}\end{array}$ & $\begin{array}{l}\text { \% } \\
\text { OT }\end{array}$ & $\begin{array}{l}\text { \% } \\
\text { E }\end{array}$ & $\begin{array}{l}\% \\
\text { L }\end{array}$ \\
\hline Date/time of test runs & & & & & & & & \\
\hline
\end{tabular}

False Alarm Incident Report

\begin{tabular}{|c|l|}
\hline $\begin{array}{c}\text { Run } \\
\#\end{array}$ & Probable Cause \\
\hline 1 & Example: shadows on road misinterpreted as road edge \\
\hline$\ldots$ & $\ldots$ \\
\hline
\end{tabular}




\subsection{Curved Road with Shoulder (Maximum AMR)}

The Curved Road with Shoulder test assesses the warning system's ability to detect the shoulder width and to adjust the warning location on a curved road departure. This test is similar to the test in section 7.1.

The figure below illustrates the test scenario. The left-side departure test uses a right turn curve.
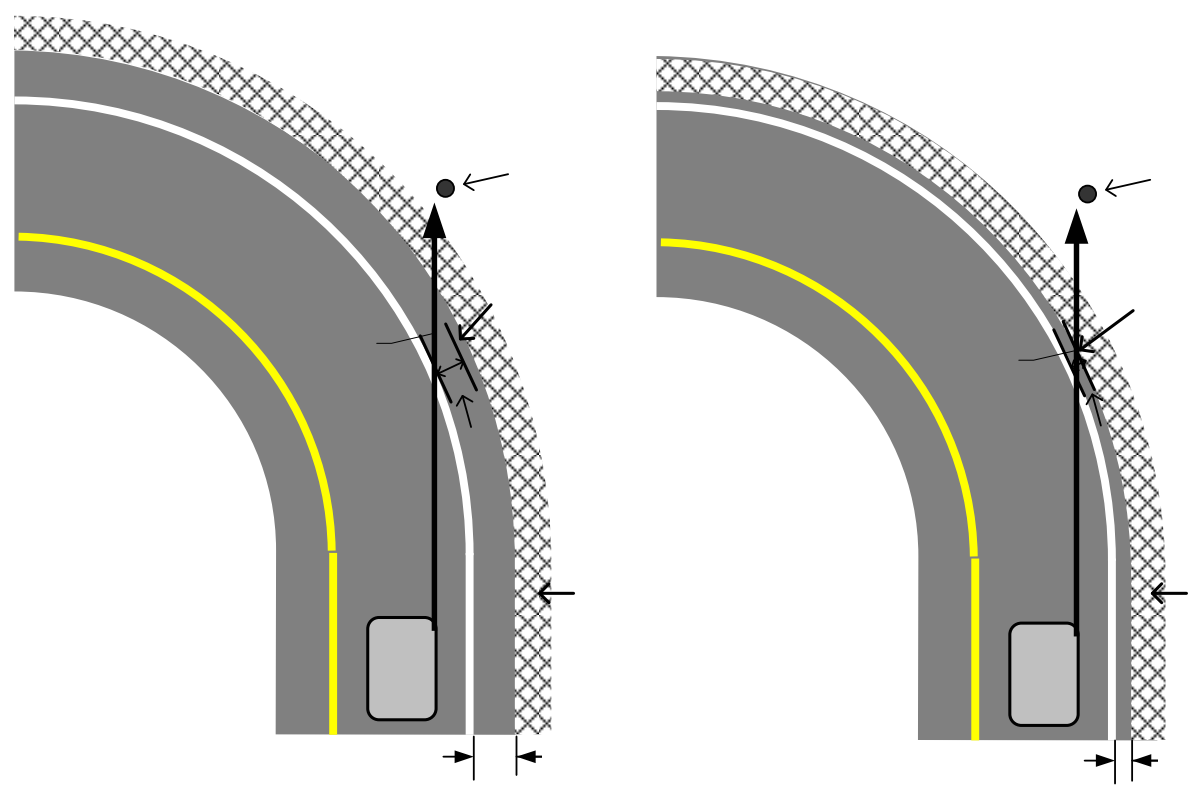

Figure 23. Curved Road with Shoulder Test Trajectory

\subsubsection{Events and System Response}

The following table defines the events for this test and the correct warning signal the system shall provide. See the figure above to locate the event.

\begin{tabular}{|c|l|l|}
\hline Id & \multicolumn{1}{|c|}{ Event } & \multicolumn{1}{c|}{ Correct Response } \\
\hline A1 & Road departure & 1. Issue road departure warning \\
\hline B1 & Same as A1 & 1. Same as A1 except earlier \\
\hline
\end{tabular}

\subsubsection{Test Conditions}

The conditions for this test include the following. 


\subsubsection{Road Geometry}

The curve radius for this test is appropriate for the low speed, medium speed and highspeed conditions. The following equation calculates the curve radius for each speed condition using lateral acceleration limits $\left(a_{\text {lat }}\right)$ from section 2.3.5.

$r=\frac{v^{2}}{a_{\text {lat }}}$

For the test, the curve radius falls within a range of radii by using the lateral acceleration limits for sensitivities 2 and 4.

\begin{tabular}{|l|l|}
\hline Road Geometry & Dimension \\
\hline Low speed curve, $64 \mathrm{~km} / \mathrm{h}(40 \mathrm{mph})$ & $91 \mathrm{~m}-136 \mathrm{~m}$ \\
\hline Medium speed curve, $89 \mathrm{~km} / \mathrm{h}(55 \mathrm{mph})$ & $172 \mathrm{~m}-257 \mathrm{~m}$ \\
\hline Low speed curve, $113 \mathrm{~km} / \mathrm{h}(70 \mathrm{mph})$ & $278 \mathrm{~m}-417 \mathrm{~m}$ \\
\hline
\end{tabular}

Appendix A, Methods for Determining Road Dimensions and Geometry, contains the recommended method to verify the geometry.

\subsubsection{Available Maneuver Room}

In this test, shoulder width affects the AMR. However, the test limits the AMR to a default maximum value to prevent large variations in warning location due to large variations in shoulder width.

\begin{tabular}{|l|l|}
\hline Available Maneuver Room & Comment \\
\hline $1 \mathrm{~m}(39 \mathrm{in})$ & Default maximum AMR \\
\hline
\end{tabular}

\subsubsection{Speed}

The vehicle speeds during the tests cover the warning system operating range.

\begin{tabular}{|l|l|l|}
\hline Low Speed & Medium Speed & High Speed \\
\hline $64 \mathrm{~km} / \mathrm{h}(40 \mathrm{mph})$ & $89 \mathrm{~km} / \mathrm{h}(55 \mathrm{mph})$ & $113 \mathrm{~km} / \mathrm{h}(70 \mathrm{mph})$ \\
\hline
\end{tabular}

\subsubsection{Shoulder Width}

This test uses two shoulder widths. The small shoulder is one half the maximum AMR and the large shoulder is twice the AMR. Update these values if the default minimum or maximum AMR changes.

\begin{tabular}{|l|l|}
\hline Shoulder Width Name & \multicolumn{1}{|c|}{ Shoulder Width Dimension } \\
\hline Small Shoulder & $0.5 \mathrm{~m}(19.5 \mathrm{in})$ \\
\hline Large Shoulder & $2 \mathrm{~m}$ \\
\hline
\end{tabular}

\subsubsection{Off-Road Material}

These tests use two types of material to simulate off-road surfaces. The operator places the material on a wide shoulder to reduce the visual width of the shoulder to the widths specified above. 


\begin{tabular}{|l|l|}
\hline Off-road & \multicolumn{1}{c|}{ Characteristics } \\
\hline Dirt & Brown or variations. \\
\hline Grass & Green artificial grass material \\
\hline
\end{tabular}

\subsubsection{Measurements}

Measurements collected during the test support the following:

1. Determine whether a warning was given

2. Determine the desired location of a warning given the conditions

3. Determine the amount of time the warning gives driver

\subsubsection{Time of Warning}

See section 2.3.9.

\subsubsection{Longitudinal Speed}

See section 2.2.2.

\subsubsection{Distance to Road Edge}

See section 2.2.7. For this test, the road edge is the base of the barrier.

\subsubsection{Distance to Curve Entry Point}

See section 2.2.10.

\subsubsection{Test Procedure}

The test repeats a series of road departure trajectories as depicted in Figure 23.

\subsubsection{Pre-Test Procedures}

The procedures before the test are:

1. Ensure road geometry meets the test condition. Measure the geometry if it is unknown (see Appendix A, Methods for Determining Road Dimensions and Geometry).

2. Ensure lane marker characteristics meet the test conditions (see sections 2.1.12.1.12).

3. Check speedometer accuracy (see section 2.2.2).

4. Verify IMS camera calibration according to recommendations in [5]. Repeat camera calibration if camera has moved.

5. Ensure microphone detects warning system alerts and driver speech.

6. Ensure recording system is working properly.

7. Ensure GPS has sufficient satellite signals.

8. Verify road boundary for test (i.e., outer lane marker plus AMR) and practice vehicle trajectories (speeds, departure rate, etc.) until accomplished safely and reliably.

9. Set the AMR width with dirt off-road material. 


\subsubsection{Test Procedures}

The procedures during the test are:

1. Achieve speed. The driver brings the vehicle to the prescribed speed. The driver may use cruise control if the vehicle is properly equipped.

2. Straighten vehicle and center vehicle in the lane before entering the curve. The driver centers the vehicle in the lane using the lane markers as a visual guide. The vehicle remains in the center until the start of the curve.

3. Maintain straight trajectory into the curve. As the vehicle enters the curve, the driver uses a guide target to maintain the straight lane trajectory through the departure.

4. Depart road. The driver crosses over the lane marker and then recovers from the departure.

5. Repeat steps 1-4 for:

a. Five right departures.

b. Five left departures.

6. Repeat steps $1-5$ for the middle speed curve.

7. Repeat steps $1-5$ for the high-speed curve.

8. Repeat steps $1-7$ with the small shoulder width.

9. Repeat steps $1-8$ with the grass off-road material.

\subsubsection{Post-Test Procedures}

The procedures after the test are:

1. Verify IMS camera orientation has not changed. Repeat tests if changed.

\subsubsection{Evaluation Methods}

Use the following methods to evaluate warning system performance.

\subsubsection{Rate System Response}

Examine the video and audio data and rate each warning scenario.

The rating is a true positive if the system responds with a warning before the road boundary is crossed.

The rating is a false negative if the system does not respond with a warning before the road boundary is crossed.

\subsubsection{Desired Location of Warning}

For each test run, the evaluator determines the desired location of the warning. The desired location varies with the departure rate and forward speed.

1. Locate the time of warning in the video data (for example, by locating video frame where alert is first displayed).

2. Measure lateral position and lateral velocity to the road edge at time of warning. 
3. Use equation (4) in section 5.1.2 to determine the desired location for a warning on a curve.

\subsubsection{Timeliness of Warning}

For each test run, the evaluator determines whether the warning is early, late or on time using steps described in section Error! Reference source not found.. The results of the timeliness evaluations are accumulated according to section 5.3.

\subsubsection{Reporting}

The evaluator completes the following tables from the data in each test.

\begin{tabular}{|l|l|}
\hline Name of Test & Departure on Curved Road with Shoulder \\
\hline Date of Test & \\
\hline Warning System ID & Name and Version Number \\
\hline
\end{tabular}

Test Run Results

\begin{tabular}{|l|l|l|l|l|l|l|c|c|c|c|}
\hline \multicolumn{9}{|c|}{ Factors } & \multicolumn{6}{|c|}{ Results } \\
\hline $\begin{array}{c}\text { Offroad } \\
\text { Mat. }\end{array}$ & Curve & $\begin{array}{c}\text { Speed } \\
\mathbf{m p h}\end{array}$ & Direction & $\begin{array}{c}\text { \# of } \\
\text { runs }\end{array}$ & $\begin{array}{c}\text { Run } \\
\#\end{array}$ & $\begin{array}{c}\mathbf{C R} \\
\mathbf{T} / \mathbf{F}\end{array}$ & $\begin{array}{c}\mathbf{v}_{\mathbf{f}} \\
\mathbf{m p h}\end{array}$ & $\begin{array}{c}\mathbf{y}_{\mathbf{m}} \\
\mathbf{m}\end{array}$ & $\begin{array}{c}\mathbf{x}_{\mathbf{w}} \\
\mathbf{m}\end{array}$ & $\begin{array}{c}\mathbf{T} \\
\mathbf{O T} / \mathbf{E} / \mathbf{L}\end{array}$ \\
\hline Dirt & LS & 40 & Left & 5 & 1 & & & & & \\
\hline$"$ & $"$ & $"$ & $"$ & & $2 \ldots$ & & & & & \\
\hline Dirt & LS & 40 & Right & 5 & & & & & & \\
\hline Dirt & MS & 55 & Left & 5 & & & & & & \\
\hline Dirt & MS & 55 & Right & 5 & & & & & & \\
\hline Dirt & HS & 70 & Left & 5 & & & & & & \\
\hline Dirt & HS & 70 & Right & 5 & & & & & & \\
\hline Grass & LS & 40 & Left & 5 & & & & & & \\
\hline Grass & LS & 40 & Right & 5 & & & & & & \\
\hline Grass & MS & 55 & Left & 5 & & & & & & \\
\hline Grass & MS & 55 & Right & 5 & & & & & & \\
\hline Grass & HS & 70 & Left & 5 & & & & & & \\
\hline Grass & HS & 70 & Right & 5 & & & & & & \\
\hline
\end{tabular}

Curve $=$ Low speed curve, medium speed curve and high speed curve

$\mathrm{CR}=$ System gave correct response $(\mathrm{T})$ or failed to give correct response $(\mathrm{F})$. Refer to subsection 1.1 above to determine correct response.

$\mathrm{v}_{\mathrm{f}}=$ vehicle forward speed

$\mathrm{y}_{\mathrm{m}}=$ the measured lateral distance between the vehicle front tire and the road boundary at the time of warning

$\mathrm{x}_{\mathrm{w}}=$ the desired distance to curve entry point at the time of warning using equation (4)

$\mathrm{T}=$ Timeliness of warning (early, late or on time)

Test Run Summary Results (see section 5.3)

\begin{tabular}{|c|c|c|c|c|c|c|c|c|}
\hline $\begin{array}{c}\text { Combined Results for } \\
\text { all runs }\end{array}$ & $\begin{array}{c}\# \text { of } \\
\text { runs }\end{array}$ & $\begin{array}{c}\# \\
\text { CR }\end{array}$ & $\begin{array}{c}\# \\
\text { OT }\end{array}$ & $\begin{array}{c}\# \\
\text { E }\end{array}$ & $\begin{array}{c}\# \\
\text { L }\end{array}$ & $\begin{array}{c}\text { \% } \\
\text { OT }\end{array}$ & $\begin{array}{c}\text { \% } \\
\text { E }\end{array}$ & $\begin{array}{c}\text { \% } \\
\text { L }\end{array}$ \\
\hline Date/time of test runs & & & & & & & & \\
\hline
\end{tabular}


False Alarm Incident Report

\begin{tabular}{|c|l|}
\hline $\begin{array}{c}\text { Run } \\
\#\end{array}$ & Probable Cause \\
\hline 1 & Example: shadows on road misinterpreted as road edge \\
\hline$\ldots$ & $\ldots$ \\
\hline
\end{tabular}




\section{Tests for Departures Toward an Obstacle}

These tests evaluate warning system response to road departures toward an obstacle on the shoulder. The warning system detects the obstacle, decreases the available maneuver room, and issues a warning based on the location of the obstacles. These tests do not assess the system's ability to determine whether the shoulder ends before the obstacle (i.e.., the obstacle is off-road). Therefore, a warning system must also pass the "Tests for Departures on Roads with Maximum AMR" that appear in section 7.

These tests evaluate the system's ability to detect an obstacle and to warn when there is a potential for a lateral collision. A map may provide information regarding obstacles, but this information may be incomplete, inaccurate or unavailable. Thus, a warning system must use a real-time sensor (e.g., radar) to detect obstacles. The warning system deals with longitudinal barriers and discrete objects.

A longitudinal barrier appears as one long continuous obstacle, such as a jersey barrier. The vehicle drifts slowly toward the barrier much like a lane departure. The warning system uses a side-looking sensor to detect these obstacles.

A discrete object appears on the shoulder ahead of the vehicle. Examples of discrete obstacles include parked cars, bridge supports, and the beginning of a longitudinal barrier. If the vehicle drifts too far outside the lane, it will collide with the object. After a warning, the driver steers away from the obstacle to avoid a collision. The system uses a side-forward sensor (i.e., aimed forward and to the side) to detect these obstacles.

The sensors used by the system must detect a wide range of obstacles with varying properties. Small obstacles, such as pedestrians and motorcycles, pose the greater challenges. The tests use similar sized obstacles made of safer material to reduce the risk of serious damage.

The tests involve placing the obstacles at two distances (i.e., offsets) from the lane edge. The large offset is twice the maximum AMR. The system should detect the large obstacle offset but limit the AMR to the maximum in order to provide consistent warnings. The small obstacle offset is half the maximum AMR. The system should detect the small offset and adjust the warning accordingly. The earlier warning indicates the system successfully detected the obstacle.

The test scenarios include obstacles on a straight road and on a curved road. 


\subsection{Barrier on Straight Road}

The Barrier on Straight Road test assesses the warning system's ability to detect the location of a barrier and to adjust the warning location on a straight road departure.

The figure below illustrates the test scenario. Left-side collision tests position the barrier outside of the yellow line.

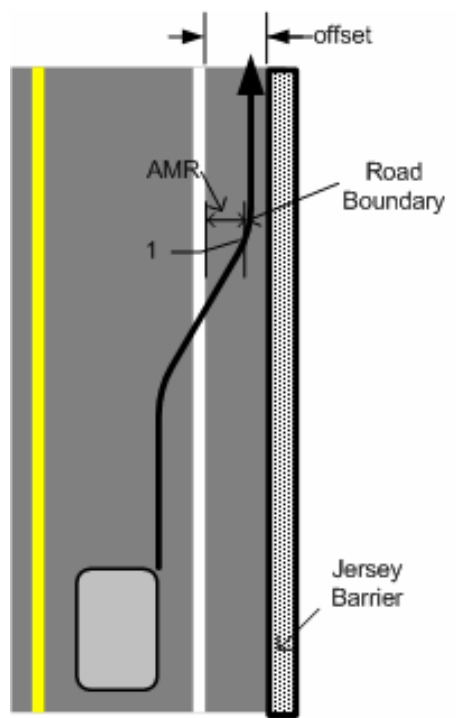

A

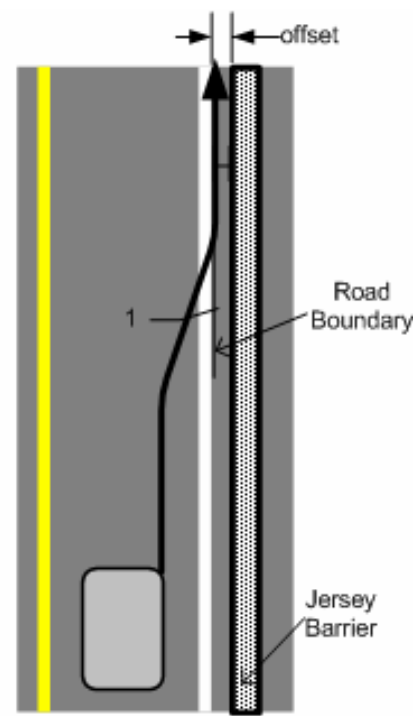

B

Figure 24. Barrier on Straight Road Test, Vehicle Trajectory

\subsubsection{Events and System Response}

The following table defines the events for this test and the correct system response. See the figure above to locate the event (e.g., Id A1 is label 1 in figure A).

\begin{tabular}{|c|l|l|}
\hline Id & \multicolumn{1}{|c|}{ Event } & \multicolumn{1}{c|}{ Correct Response } \\
\hline A1 & Lateral collision & 1. Issue lateral collision warning \\
\hline B1 & Lateral collision & 1. Same as A1 \\
\hline
\end{tabular}

\subsubsection{Test Conditions}

The conditions for this test include the following.

\subsubsection{Road Geometry}

The road geometry for the test is: 


\begin{tabular}{|l|l|}
\hline Road Geometry & Dimension \\
\hline Straight (defined using radius of curvature) & $>1000 \mathrm{~m}$ \\
\hline
\end{tabular}

Appendix A, Methods for Determining Road Dimensions and Geometry, contains the recommended method to verify the road geometry.

\subsubsection{Available Maneuver Room}

In this test, obstacle offset affects the AMR. However, the test limits the AMR to a default maximum value to prevent large variations in warning location due to large variations in obstacle offset.

\begin{tabular}{|l|l|}
\hline Available Maneuver Room & Comment \\
\hline $1 \mathrm{~m}(39 \mathrm{in})$ & Maximum AMR \\
\hline
\end{tabular}

\subsubsection{Speed}

The vehicle speeds during the tests cover the warning system operating range.

\begin{tabular}{|l|l|l|}
\hline Low Speed & Medium Speed & High Speed \\
\hline $64 \mathrm{~km} / \mathrm{h}(40 \mathrm{mph})$ & $89 \mathrm{~km} / \mathrm{h}(55 \mathrm{mph})$ & $113 \mathrm{~km} / \mathrm{h}(70 \mathrm{mph})$ \\
\hline
\end{tabular}

\subsubsection{Departure rate}

The vehicle departure rates during the tests fall within two ranges. These rates are comparable to the rates suggested in the ROR report and in the ISO standard for testing lateral drift warning systems.

\begin{tabular}{|l|l|}
\hline Low Departure Rate & High Departure Rate \\
\hline$\leq 0.75 \mathrm{~m} / \mathrm{s}$ & $>0.75 \mathrm{~m} / \mathrm{s}$ \\
\hline
\end{tabular}

\subsubsection{Barrier}

For safety purposes, this test uses a plastic, water-filled jersey barrier as shown in Figure 25. 


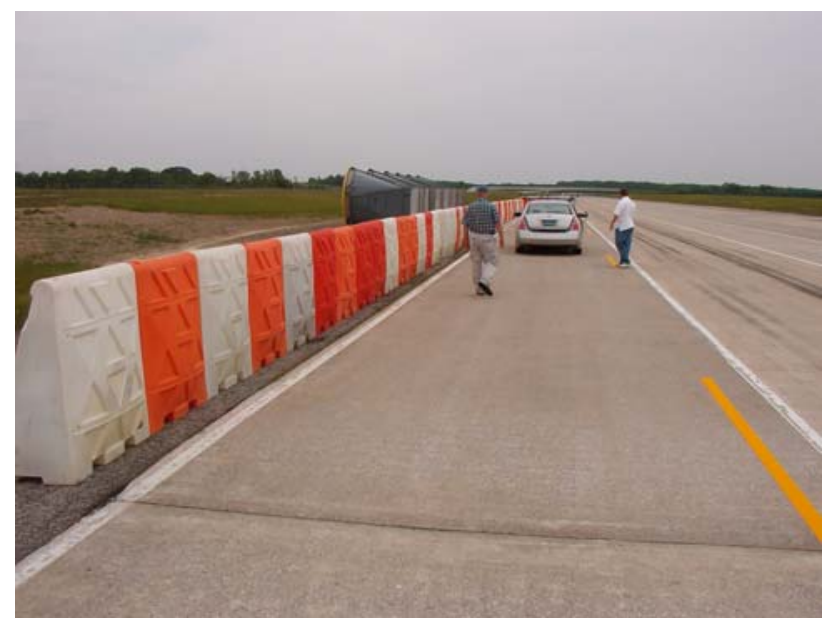

Figure 25. Water-filled Plastic Jersey Barrier

\subsubsection{Barrier Offset}

The tests involve placing the obstacles at two offsets measured from the outside edge of the lane marker. The small offset is one half the maximum AMR and the large offset is twice the AMR. The system evaluator updates these values if the default minimum or maximum AMR changes.

\begin{tabular}{|l|l|}
\hline Barrier Offset Name & \multicolumn{1}{c|}{ Dimension } \\
\hline Small Offset & $0.5 \mathrm{~m}(19.5 \mathrm{in})$ \\
\hline Large Offset & $2 \mathrm{~m}(79 \mathrm{in})$ \\
\hline
\end{tabular}

\subsubsection{Measurements}

Measurements collected during the test support the following:

1. Determine whether a warning was given

2. Determine the desired location of a warning given the conditions

3. Determine the amount of time the warning gives driver

\subsubsection{Time of Warning}

See section 2.3.9.

\subsubsection{Longitudinal Speed}

See section 2.2.2.

\subsubsection{Distance to Road Edge}

See section 2.2.7. For this test, the road edge is the base of the barrier.

\subsubsection{Lateral Velocity}

See section 2.2.4. 


\subsubsection{Test Procedure}

The general procedure for the test repeats a series of road departure trajectories depicted in Figure 24.

\subsubsection{Pre-Test Procedures}

The procedures before the test are:

1. Ensure road geometry meets the test condition and measure the geometry if unknown (see Appendix A, Methods for Determining Road Dimensions and Geometry).

2. Ensure lane marker characteristics meet the test conditions and measure the characteristics if it is unknown (see sections 2.1.1-2.1.12).

3. Check speedometer accuracy (see section 2.2.2).

4. Verify IMS camera calibration according to recommendations in [5] and repeat camera calibration if camera has moved.

5. Ensure microphone detects warning system alerts and driver speech.

6. Ensure recording system is working properly.

7. Ensure GPS has sufficient satellite signals.

8. Assemble the barriers at the desired offset (large distance for first test set).

9. Verify road boundary for test (i.e., outer lane marker plus AMR) and practice vehicle trajectories (speeds, departure rate, etc.) until accomplished safely and reliably.

\subsubsection{Test Procedures}

The procedures during the test are:

1. Achieve speed. The driver brings the vehicle to the prescribed speed. The driver may use cruise control if the vehicle is properly equipped.

2. Execute departure. The driver steers toward the barrier at the prescribed departure rate (high or low) until a warning occurs or the driver decides to avoid the barrier. Section 2.2.8 provides a method to achieve the prescribed departure rate.

3. Record departure rate. The assistant records the estimated departure rate as either low or high.

4. Go to start of next collision trajectory. The driver brings the vehicle back around in preparation for the next collision trajectory.

5. Repeat steps 1-4 for:

a. Five right-side collisions at the low departure rate.

b. Five left-side collisions at the low departure rate.

c. Five right-side collisions at the high departure rate.

d. Five left-side collisions at the high departure rate.

6. Repeat steps $1-5$ for the prescribed middle speed.

7. Repeat steps $1-5$ for the prescribed high speed.

8. Repeat steps $1-7$ with the barriers at the small offset.

\subsubsection{Post-Test Procedures}


The procedures after the test are:

1. Verify IMS camera orientation has not changed. Repeat tests if changed.

\subsubsection{Evaluation Methods}

Use the following methods to evaluate warning system performance.

\subsubsection{Rate System Response}

Examine the video and audio data and rate each warning scenario.

The rating is a true positive if the system responds with a warning before the road boundary is crossed.

The rating is a false negative if the system does not respond with a warning before the road boundary is crossed.

\subsubsection{Desired Location of Warning}

For each test run, the evaluator determines the desired location of the warning. The desired location varies with the forward speed and curve radii.

1. Locate the time of warning in the video data (for example, by locating video frame where alert is first displayed).

2. Measure lateral position and lateral velocity to the road edge at time of warning.

3. Use equation (1) in section 5.1.1 to determine the desired location for a warning on a straight road.

\subsubsection{Timeliness of Warning}

For each test run, the evaluator determines whether the warning is early, late or on time using steps described in section 5.1.1. The results of the timeliness evaluations are accumulated according to section 5.3.

\subsubsection{Reporting}

The evaluator completes the following tables from the data in each test.

\begin{tabular}{|l|l|}
\hline Name of Test & Collision with Barrier on Straight Road \\
\hline Date of Test & \\
\hline Warning System ID & Name and Version Number \\
\hline
\end{tabular}

Test Run Results

\begin{tabular}{|c|c|c|c|c|c|c|c|c|c|c|c|c|}
\hline \multicolumn{6}{|c|}{ Factors } & \multicolumn{7}{|c|}{ Results } \\
\hline $\begin{array}{c}\text { Offset } \\
\text { m }\end{array}$ & $\begin{array}{c}\begin{array}{c}\text { Speed } \\
\text { mph }\end{array} \\
\end{array}$ & $\begin{array}{c}\text { Departure } \\
\text { Rate } \mathrm{m} / \mathrm{s}\end{array}$ & Direction & Signal & $\begin{array}{l}\text { \# of } \\
\text { runs }\end{array}$ & $\begin{array}{c}\text { Run } \\
\#\end{array}$ & $\begin{array}{l}\text { CR } \\
\text { T/F }\end{array}$ & $\begin{array}{c}\mathbf{v}_{\mathrm{f}} \\
\mathrm{mph}\end{array}$ & $\begin{array}{l}\mathbf{v}_{\mathbf{l}} \\
\mathbf{m} / \mathbf{s}\end{array}$ & $\begin{array}{c}\mathbf{y}_{\mathbf{m}} \\
\mathrm{m}\end{array}$ & $\begin{array}{c}\mathbf{y}_{\mathrm{w}} \\
\mathbf{m}\end{array}$ & $\begin{array}{c}\mathbf{T} \\
\text { OT/E/L }\end{array}$ \\
\hline 0.5 & 40 & $\leq 0.75$ & Left & No & 5 & 1 & & & & & & \\
\hline 0.5 & $"$ & " & " & $"$ & & $2 \ldots$ & & & & & & \\
\hline 0.5 & 40 & $\leq 0.75$ & Right & No & 5 & & & & & & & \\
\hline
\end{tabular}




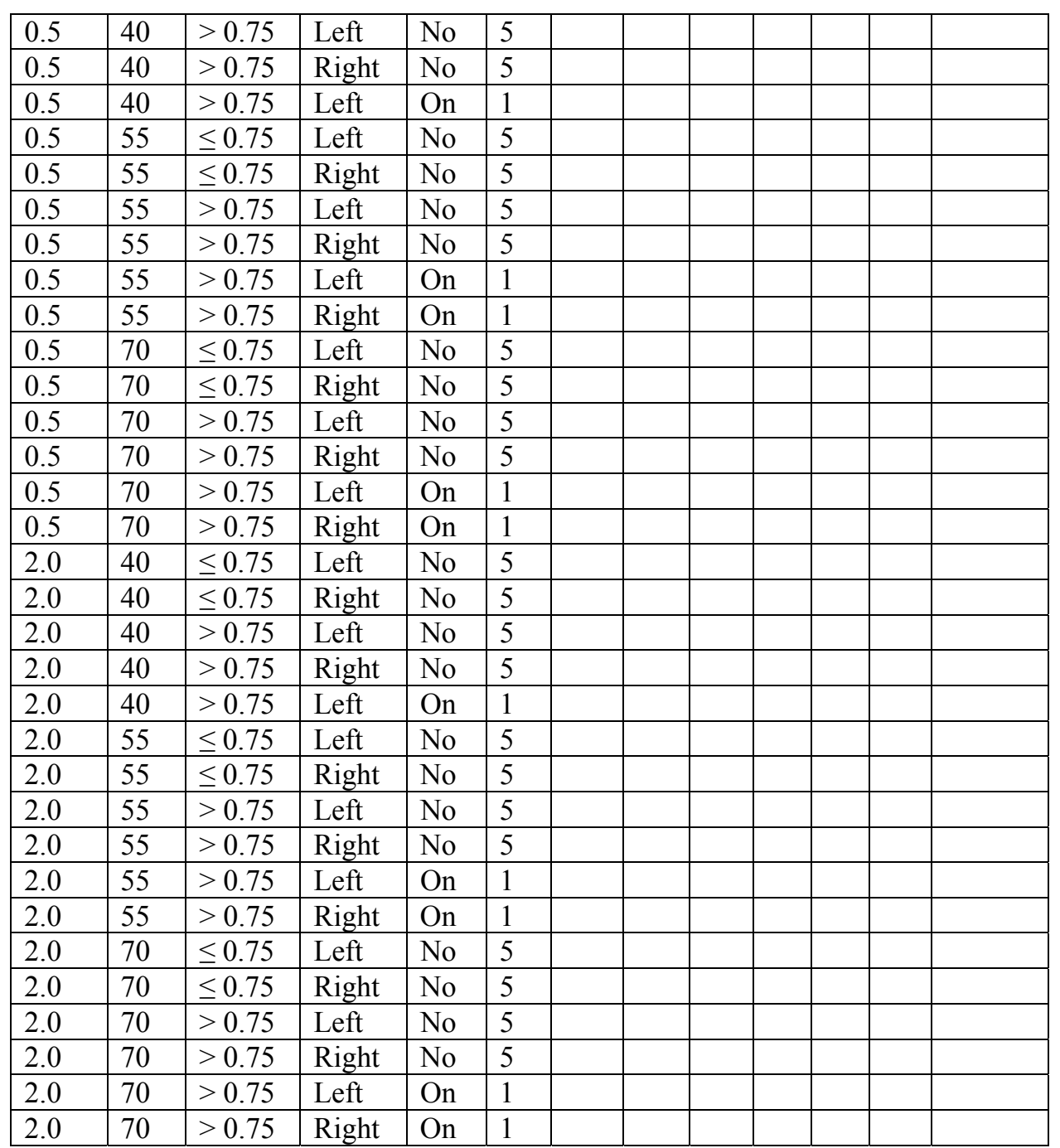

Offset $=$ Offset distance (in meters) of barrier from outside edge of lane marker

$\mathrm{CR}=$ System gave correct response $(\mathrm{T})$ or failed to give correct response (F). Refer to subsection 1.1 above to determine correct response.

$\mathrm{v}_{\mathrm{f}}=$ measured vehicle forward speed

$\mathrm{v}_{\mathrm{l}}=$ measured vehicle lateral speed (departure rate)

$\mathrm{y}_{\mathrm{m}}=$ measured lateral distance between the vehicle front tire and the road boundary at the time of warning

$\mathrm{y}_{\mathrm{w}}=$ the desired lateral distance at the time of warning using equation (1)

$\mathrm{T}=$ Timeliness of warning (early, late or on time)

Test Run Summary Results (see section 5.3)

\begin{tabular}{|c|c|c|c|c|c|c|c|c|}
\hline $\begin{array}{l}\text { Combined Results for } \\
\text { all runs }\end{array}$ & $\begin{array}{l}\# \text { of } \\
\text { runs }\end{array}$ & $\begin{array}{c}\# \\
\text { CR }\end{array}$ & $\begin{array}{c}\# \\
\text { OT }\end{array}$ & $\mathbf{E}$ & $\mathbf{L}$ & $\begin{array}{c}\% \\
\text { O1 }\end{array}$ & 0 & $\%$ \\
\hline
\end{tabular}




\begin{tabular}{|l|l|l|l|l|l|l|l|l|}
\hline Date/time of test runs & & & & & & & & \\
\hline
\end{tabular}

False Alarm Incident Report

\begin{tabular}{|c|l|}
\hline $\begin{array}{c}\text { Run } \\
\#\end{array}$ & Probable Cause \\
\hline 1 & Example: shadows on road misinterpreted as road edge \\
\hline$\ldots$ & $\ldots$ \\
\hline
\end{tabular}




\subsection{Barrier on Curved Road}

The Barrier on Curved Road test assesses the warning system's ability to detect the location of a barrier and to adjust the warning location on a curved road departure.

The figure below illustrates the test scenario. The left-side collision avoidance test uses a right turn curve.

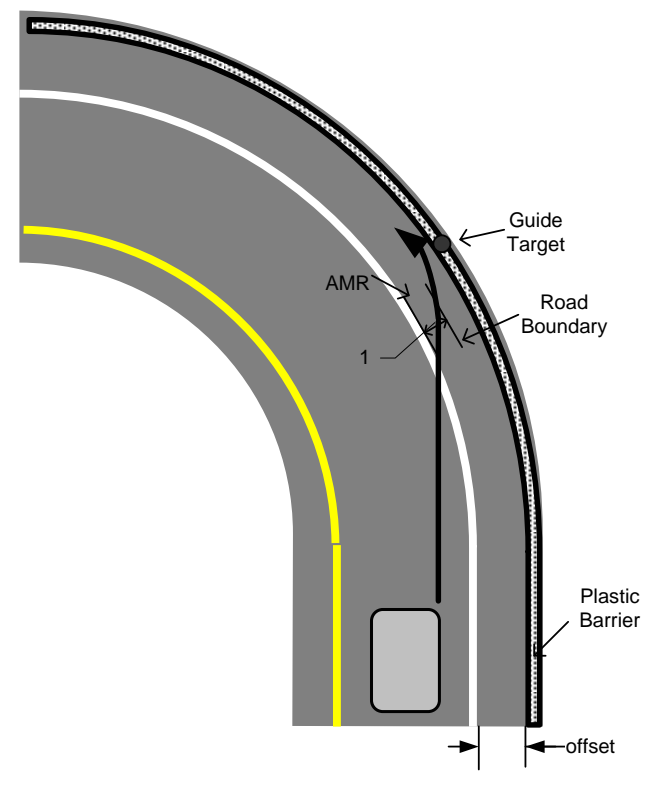

A

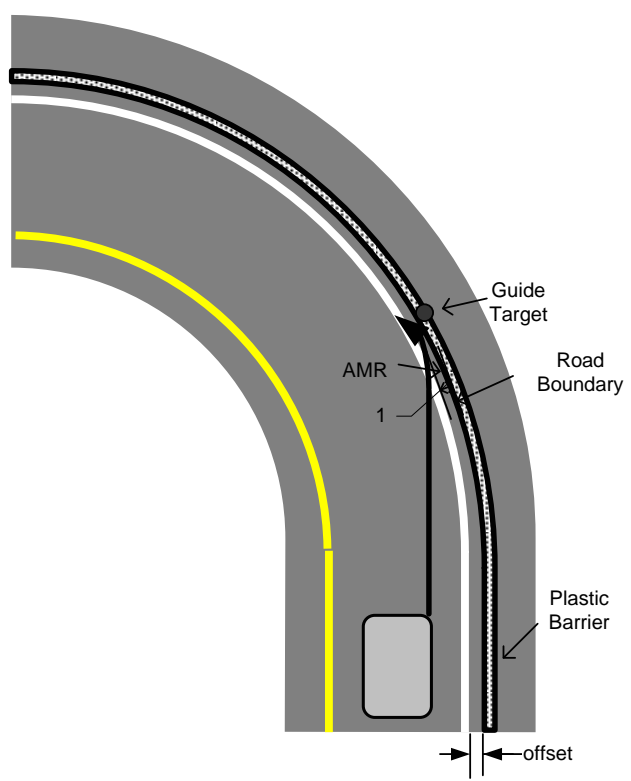

B

Figure 26. Barrier on Curved Road Test Vehicle Trajectory

\subsubsection{Events and System Response}

The following table defines the events for this test and the correct system response. See the figure above to locate the event (e.g., Id A1 is label 1 in figure A).

\begin{tabular}{|c|l|l|}
\hline Id & \multicolumn{1}{|c|}{ Event } & \multicolumn{1}{c|}{ Correct Response } \\
\hline A1 & Lateral collision & 1. Issue lateral collision warning \\
\hline B1 & Lateral collision & 1. Same as A1 except earlier \\
\hline
\end{tabular}

\subsubsection{Test Conditions}

The conditions for this test include the following. 


\subsubsection{Road Geometry}

The curve radius for this test should be appropriate for the low speed, medium speed and high-speed conditions. The following equation calculates the curve radius for each speed condition using lateral acceleration limits $\left(a_{\text {lat }}\right)$ from section 2.3.5.

$r=\frac{v^{2}}{a_{\text {lat }}}$

For the test, the curve radius falls within a range of radii by using the lateral acceleration limits for sensitivities 2 and 4.

\begin{tabular}{|l|l|}
\hline Road Geometry & Dimension \\
\hline Low speed curve, $64 \mathrm{~km} / \mathrm{h}(40 \mathrm{mph})$ & $91 \mathrm{~m}-136 \mathrm{~m}$ \\
\hline Medium speed curve, $89 \mathrm{~km} / \mathrm{h}(55 \mathrm{mph})$ & $172 \mathrm{~m}-257 \mathrm{~m}$ \\
\hline Low speed curve, $113 \mathrm{~km} / \mathrm{h}(70 \mathrm{mph})$ & $278 \mathrm{~m}-417 \mathrm{~m}$ \\
\hline
\end{tabular}

Appendix A, Methods for Determining Road Dimensions and Geometry, contains the recommended method to verify the road geometry.

\subsubsection{Available Maneuver Room}

In this test, obstacle offset affects the AMR. However, the test limits the AMR to a default maximum value to prevent large variations in warning location due to large variations in obstacle offset.

\begin{tabular}{|l|l|}
\hline Available Maneuver Room & Comment \\
\hline $1 \mathrm{~m}(39$ in $)$ & Maximum AMR \\
\hline
\end{tabular}

\subsubsection{Speed}

The vehicle speeds during the tests cover the warning system operating range.

\begin{tabular}{|l|l|l|}
\hline Low Speed & Medium Speed & High Speed \\
\hline $64 \mathrm{~km} / \mathrm{h}(40 \mathrm{mph})$ & $89 \mathrm{~km} / \mathrm{h}(55 \mathrm{mph})$ & $113 \mathrm{~km} / \mathrm{h}(70 \mathrm{mph})$ \\
\hline
\end{tabular}

\subsubsection{Barrier}

For safety purposes, this test uses a plastic, water-filled jersey barrier (see Figure 25).

\subsubsection{Barrier Offset}

The tests involve placing the obstacles at two offsets measured from the outside edge of the lane marker. The small offset is one half the maximum AMR and the large offset is twice the AMR. The test operator updates these values if the default minimum or maximum AMR changes.

\begin{tabular}{|l|l|}
\hline Barrier Offset Name & \multicolumn{1}{c|}{ Dimension } \\
\hline Small Offset & $0.5 \mathrm{~m}(19.5 \mathrm{in})$ \\
\hline Large Offset & $2 \mathrm{~m}(79 \mathrm{in})$ \\
\hline
\end{tabular}




\subsubsection{Measurements}

Measurements collected during the test support the following:

1. Determine whether a warning was given

2. Determine the desired location of a warning given the conditions

3. Determine the amount of time the warning gives driver

\subsubsection{Time of Warning}

See section 2.3.9.

\subsubsection{Longitudinal Speed}

See section 2.2.2.

\subsubsection{Distance to Road Edge}

See section 2.2.7. For this test, the road edge is the base of the barrier.

\subsubsection{Distance to Curve Entry Point}

See section 2.2.10.

\subsubsection{Test Procedure}

The general procedure for the test repeats a series of road departure trajectories depicted in Figure 26.

\subsubsection{Pre-Test Procedures}

The procedures before the test are:

1. Ensure road geometry meets the test condition. The operator measures the road geometry as per Appendix A if required.

2. Ensure the lane marker characteristics meet the test conditions. The operator measures the characteristics if it is unknown (see sections 2.1.1-2.1.12).

3. Check speedometer accuracy (see section 2.2.2).

4. Verify IMS camera calibration according to recommendations in [5]. Repeat camera calibration if camera has moved.

5. Ensure microphone detects warning system alerts and driver speech.

6. Ensure recording system is working properly.

7. Ensure the GPS has sufficient satellites and operates properly.

8. Assemble the barriers and position for test (use large offset for first test set).

9. Verify road boundary for test (i.e., outer lane marker plus AMR) and practice vehicle trajectories (speeds, departure rate, etc.) until accomplished safely and reliably.

\subsubsection{Test Procedures}

The procedures during the test are: 
1. Achieve speed. The driver brings the vehicle to the prescribed speed (low speed curve is $64 \mathrm{~km} / \mathrm{h}(40 \mathrm{mph}))$. The driver may use cruise control if the vehicle is properly equipped.

2. Straighten and center vehicle before entering curve. The driver centers the vehicle with the lane markers as a visual guide. The vehicle remains in the center until the start of the curve.

3. Maintain straight trajectory into the curve. As the vehicle enters the curve, the driver uses a guide target to maintain a straight trajectory toward the collision.

4. Collide with obstacle. The driver continues straight until a warning takes place or the driver aborts before a collision.

5. Repeat steps 1-4 for:

a. Five right-side collisions.

b. Five left-side collisions.

6. Repeat steps 1 - 5 for the collision on the middle speed curve.

7. Repeat steps $1-5$ for the collision on the high-speed curve.

8. Repeat steps $1-7$ with the obstacles at the small offset.

\subsubsection{Post-Test Procedures}

The procedures after the test are:

1. Verify IMS camera orientation has not changed. Repeat tests if changed.

\subsubsection{Evaluation Methods}

Use the following methods to evaluate warning system performance.

\subsubsection{Rate System Response}

Examine the video and audio data and rate each warning scenario.

The rating is a true positive if the system responds with a warning before the road boundary is crossed.

The rating is a false negative if the system does not respond with a warning before the road boundary is crossed.

\subsubsection{Desired Location of Warning}

For each test run, the evaluator determines the desired location of the warning. The desired location varies with the forward speed and curve radii.

1. Locate the time of warning in the video data (for example, by locating video frame where alert is first displayed).

2. Measure lateral position and lateral velocity to the road edge at time of warning.

3. Use equation (4) in section 5.1.2 to determine the desired location for a warning on a curve. 


\subsubsection{Timeliness of Warning}

The evaluator determines the timeliness of each warning (early, late or on time) and compiles statistics according to section 5.3.

\subsubsection{Reporting}

The evaluator completes the following tables from the data in each test.

\begin{tabular}{|l|l|}
\hline Name of Test & Collision with Barrier on Curved Road \\
\hline Date of Test & \\
\hline Warning System ID & Name and Version Number \\
\hline
\end{tabular}

Test Run Results

\begin{tabular}{|l|l|l|l|l|l|l|l|l|l|l|}
\hline \multicolumn{9}{|c|}{ Factors } & \multicolumn{7}{|c|}{ Results } \\
\hline $\begin{array}{c}\text { Offset } \\
\mathbf{m}\end{array}$ & Curve & $\begin{array}{c}\text { Speed } \\
\mathbf{m p h}\end{array}$ & Direction & $\begin{array}{c}\text { \# of } \\
\text { runs }\end{array}$ & $\begin{array}{c}\text { Run } \\
\#\end{array}$ & $\begin{array}{c}\text { CR } \\
\mathbf{T} / \mathbf{F}\end{array}$ & $\begin{array}{c}\mathbf{v}_{\mathbf{f}} \\
\mathbf{m p h}\end{array}$ & $\begin{array}{c}\mathbf{y}_{\mathbf{m}} \\
\mathbf{m}\end{array}$ & $\begin{array}{c}\mathbf{x}_{\mathbf{w}} \\
\mathbf{m}\end{array}$ & $\begin{array}{c}\mathbf{T} \\
\text { OT/E/L }\end{array}$ \\
\hline 0.075 & LS & 40 & Left & 5 & 1 & & & & & \\
\hline$"$ & $"$ & $"$ & $"$ & & $2 \ldots$ & & & & & \\
\hline 0.075 & LS & 40 & Right & 5 & & & & & & \\
\hline 0.075 & MS & 55 & Left & 5 & & & & & & \\
\hline 0.075 & MS & 55 & Right & 5 & & & & & & \\
\hline 0.075 & HS & 70 & Left & 5 & & & & & & \\
\hline 0.075 & HS & 70 & Right & 5 & & & & & & \\
\hline 2.0 & LS & 40 & Left & 5 & & & & & & \\
\hline 2.0 & LS & 40 & Right & 5 & & & & & & \\
\hline 2.0 & MS & 55 & Left & 5 & & & & & & \\
\hline 2.0 & MS & 55 & Right & 5 & & & & & & \\
\hline 2.0 & HS & 70 & Left & 5 & & & & & & \\
\hline 2.0 & HS & 70 & Right & 5 & & & & & & \\
\hline
\end{tabular}

Offset $=$ Offset distance (in meters) of barrier from outside edge of lane marker

Curve $=$ Low speed curve, medium speed curve and high speed curve

$\mathrm{CR}=$ System gave correct response $(\mathrm{T})$ or failed to give correct response $(\mathrm{F})$. Refer to subsection 1.1 above to determine correct response.

$\mathrm{v}_{\mathrm{f}}=$ vehicle forward speed

$\mathrm{y}_{\mathrm{m}}=$ the measured lateral distance between the vehicle front tire and the road boundary at the time of warning

$\mathrm{x}_{\mathrm{W}}=$ the desired distance to curve entry point at the time of warning using equation (4)

$\mathrm{T}=$ Timeliness of warning (early, late or on time)

Test Run Summary Results (see section 5.3)

\begin{tabular}{|c|c|c|c|c|c|c|c|c|}
\hline $\begin{array}{c}\text { Combined Results for } \\
\text { all runs }\end{array}$ & $\begin{array}{c}\# \text { of } \\
\text { runs }\end{array}$ & $\begin{array}{c}\# \\
\text { CR }\end{array}$ & $\begin{array}{c}\# \\
\text { OT }\end{array}$ & $\begin{array}{c}\# \\
\text { E }\end{array}$ & $\begin{array}{c}\# \\
\text { L }\end{array}$ & $\begin{array}{c}\text { O } \\
\text { OT }\end{array}$ & $\begin{array}{c}\text { \% } \\
\text { E }\end{array}$ & $\begin{array}{c}\text { \% } \\
\text { L }\end{array}$ \\
\hline Date/time of test runs & & & & & & & & \\
\hline
\end{tabular}

False Alarm Incident Report

Run Probable Cause 


\begin{tabular}{|c|l|}
\hline$\#$ & \\
\hline 1 & Example: shadows on road misinterpreted as road edge \\
\hline$\ldots$ & $\ldots$ \\
\hline
\end{tabular}




\subsection{Obstacle on Shoulder on Straight Road}

This test assesses the warning system's ability to detect an obstacle on the shoulder ahead of the vehicle and to adjust the warning location on a straight road departure.

The figure below illustrates the test scenario. Reverse the vehicle's direction to test a left-side collision.

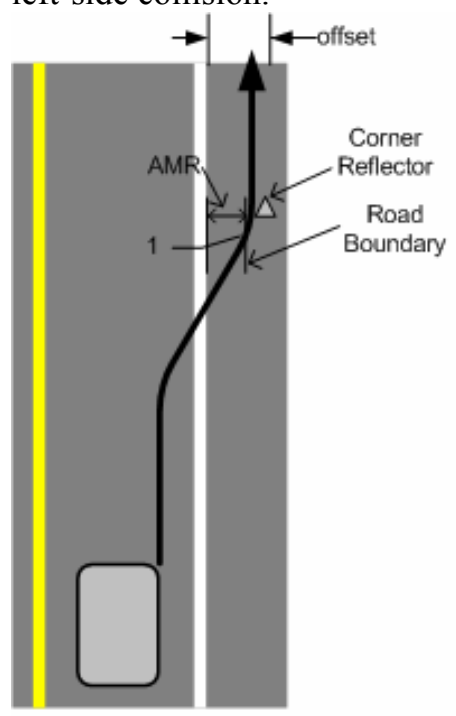

A

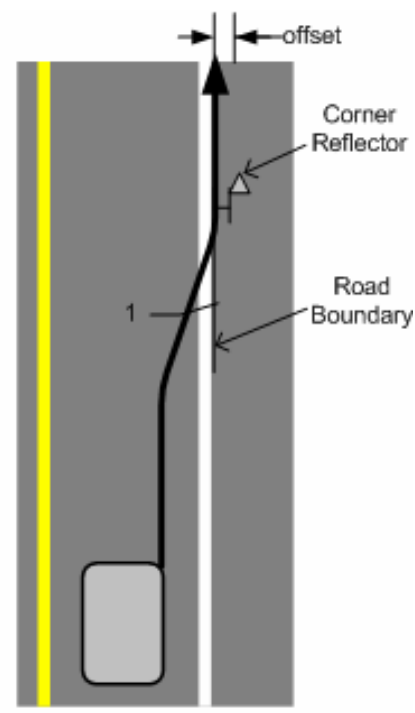

B

Figure 27. Obstacle on Shoulder on Straight Road Test, Vehicle Trajectory

\subsubsection{Events and System Response}

The following table defines the events for this test and the correct system response. See the figure above to locate the event (e.g., Id A1 is label 1 in figure A).

\begin{tabular}{|c|l|l|}
\hline Id & \multicolumn{1}{|c|}{ Event } & \multicolumn{1}{c|}{ Correct Response } \\
\hline A1 & Lateral collision & 1. Issue lateral collision warning \\
\hline B1 & Lateral collision & 1 . Same as A1 except earlier \\
\hline
\end{tabular}

\subsubsection{Test Conditions}

The conditions for this test include the following.

\subsubsection{Road Geometry}

The road geometry for the test is:

\begin{tabular}{|l|l|}
\hline Road Geometry & Dimension \\
\hline Straight (defined using radius of curvature) & $>1000 \mathrm{~m}$ \\
\hline
\end{tabular}


Appendix A, Methods for Determining Road Dimensions and Geometry, contains the recommended method to verify the road geometry.

\subsubsection{Available Maneuver Room}

In this test, obstacle offset determines the AMR. However, the AMR is limited to a maximum value to prevent large variations warning location caused by large variations shoulder width.

\section{Available Maneuver Room} $1 \mathrm{~m}(39 \mathrm{in})$

\section{Comment}

Maximum AMR

\subsubsection{Speed}

The vehicle speeds during the tests cover the warning system operating range.

\begin{tabular}{|l|l|l|}
\hline Low Speed & Medium Speed & High Speed \\
\hline $64 \mathrm{~km} / \mathrm{h}(40 \mathrm{mph})$ & $89 \mathrm{~km} / \mathrm{h}(55 \mathrm{mph})$ & $113 \mathrm{~km} / \mathrm{h}(70 \mathrm{mph})$ \\
\hline
\end{tabular}

\subsubsection{Departure rate}

The vehicle departure rates during the tests fall within two ranges. These rates are comparable to the rates suggested in the ROR report and in the ISO standard for testing lateral drift warning systems.

\begin{tabular}{|l|l|}
\hline Low Departure Rate & High Departure Rate \\
\hline$\leq 0.75 \mathrm{~m} / \mathrm{s}$ & $>0.75 \mathrm{~m} / \mathrm{s}$ \\
\hline
\end{tabular}

\subsubsection{Obstacle}

For safety purposes, this test uses a plastic, traffic barrel (see Figure 28).

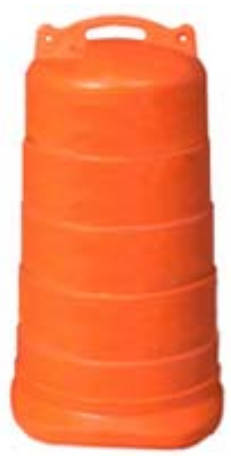

Figure 28. Traffic barrel 


\subsubsection{Obstacle Offset}

The tests involve placing the obstacles at two offsets measured from the outside edge of the lane marker. The small offset is one half the maximum AMR and the large offset is twice the AMR. The operator updates these values if the default minimum or maximum AMR changes..

\begin{tabular}{|l|l|}
\hline Barrier Offset Name & \multicolumn{1}{c|}{ Dimension } \\
\hline Small Offset & $0.5 \mathrm{~m}(19.5 \mathrm{in})$ \\
\hline Large Offset & $2 \mathrm{~m}(79 \mathrm{in})$ \\
\hline
\end{tabular}

\subsubsection{Measurements}

Measurements collected during the test support the following:

1. Determine whether a warning was given

2. Determine the desired location of a warning given the conditions

3. Determine the amount of time the warning gives driver

\subsubsection{Time of Warning}

See section 2.3.9.

\subsubsection{Longitudinal Speed}

See section 2.2.2.

\subsubsection{Distance to Road Edge}

See section 2.2.7. For this test, the base of the obstacle defines the road edge. The obstacle will not be adjacent to the front wheel at the time of warning. The evaluator calculates the distance to the road edge using the distance to the lane marker plus the obstacle offset (see figure below). To take into account the departure angle ( $\theta$ requires departure rate, see section 2.2.9), the evaluator uses the following equation (see Figure 29):

$y_{w}=l+h=l+\frac{o}{\cos \theta}$

Where:

$y_{w}=$ the lateral distance between the vehicle front tire and the road boundary

$l=$ the measured lateral distance to the lane marker

$o=$ the known offset of the obstacle

$\theta=$ the departure angle 


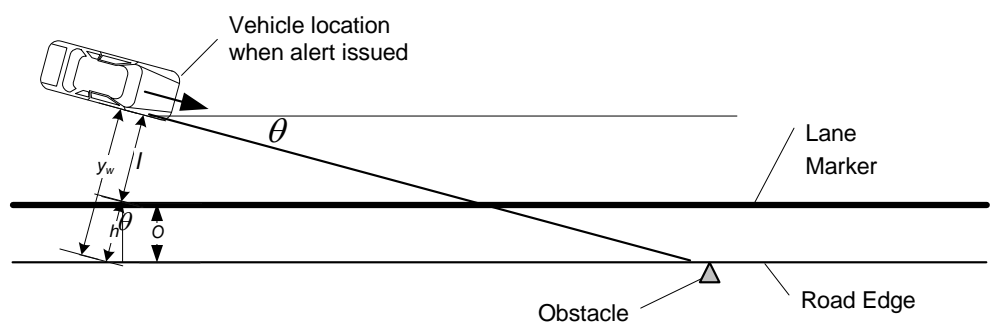

Figure 29. Indirect Distance to Road Edge

\subsubsection{Lateral Velocity}

See section 2.2.4.

\subsubsection{Test Procedure}

The general procedure for the test repeats a series of road departure trajectories depicted in Figure 27.

\subsubsection{Pre-Test Procedures}

The procedures before the test consist of the following steps:

1. Ensure road geometry meets the test condition and measure the geometry if unknown (see Appendix A, Methods for Determining Road Dimensions and Geometry).

2. Ensure lane marker characteristics meet the test conditions and measure the characteristics if unknown (see sections 2.1.1-2.1.12).

3. Check speedometer accuracy (see section 2.2.2).

4. Verify IMS camera calibration according to recommendations in [5]. Repeat camera calibration if camera has moved.

5. Ensure microphone detects warning system alerts and driver speech.

6. Ensure recording system is working properly.

7. Ensure GPS has sufficient satellite signals.

8. Assemble the barrels at the desired offset (large distance for first test set).

9. Verify road boundary for test (i.e., outer lane marker plus AMR) and practice vehicle trajectories (speeds, departure rate, etc.) until accomplished safely and reliably.

\subsubsection{Test Procedures}

The procedures during the test are:

1. Achieve speed. The driver brings the vehicle to the prescribed speed. The driver may use cruise control if the vehicle is properly equipped.

2. Execute departure. The driver steers toward the obstacle at the prescribed departure rate (high or low) until a warning occurs or the driver decides to avoid the obstacle. Section 2.2.8 provides a method to achieve the prescribed departure rate. 
3. Go to start of next collision trajectory. The driver brings the vehicle back around in preparation for the next collision trajectory.

4. Repeat steps 1-3 for:

a. Five right-side collisions at the low departure rate.

b. Five left-side collisions at the low departure rate.

c. Five right-side collisions at the high departure rate.

d. Five left-side collisions at the high departure rate.

5. Repeat steps 1 - 4 for the prescribed middle speed.

6. Repeat steps $1-4$ for the prescribed high speed.

7. Repeat steps $1-6$ with the barriers at the large offset.

\subsubsection{Post-Test Procedures}

The procedures after the test are:

1. Verify IMS camera orientation has not changed. Repeat tests if changed.

\subsubsection{Evaluation Methods}

Use the following methods to evaluate warning system performance.

\subsubsection{Rate System Response}

Examine the video and audio data and rate each warning scenario.

The rating is a true positive if the system responds with a warning before the road boundary is crossed.

The rating is a false negative if the system does not respond with a warning before the road boundary is crossed.

\subsubsection{Desired Location of Warning}

For each test run, the evaluator determines the desired location of the warning. This will vary depending on the measured departure rate and forward speed.

1. Locate the time of warning in the video data (for example, by locating video frame where alert is first displayed).

2. Measure lateral position and lateral velocity to the road edge at time of warning.

3. Use equation (1) in section 5.1.1 to determine the desired location for a warning on a straight road.

\subsubsection{Timeliness of Warning}

For each test run, the evaluator determines whether the warning is early, late or on time using steps described in section 5.1.1. The results of the timeliness evaluations are accumulated according to section 5.3.

\subsubsection{Reporting}

The evaluator completes the following tables from the data in each test. 


\begin{tabular}{|l|l|}
\hline Name of Test & Collision with Obstacle on Shoulder on Straight Road \\
\hline Date of Test & \\
\hline Warning System ID & Name and Version Number \\
\hline
\end{tabular}

Test Run Results

\begin{tabular}{|c|c|c|c|c|c|c|c|c|c|c|c|c|}
\hline & & Fact & & & & & & & $\operatorname{Res}$ & ults & & \\
\hline $\begin{array}{c}\text { Offset } \\
\text { m }\end{array}$ & $\begin{array}{c}\begin{array}{c}\text { Speed } \\
\text { mph }\end{array} \\
\text {. }\end{array}$ & $\begin{array}{c}\text { Departure } \\
\text { Rate } \mathrm{m} / \mathrm{s}\end{array}$ & \begin{tabular}{|l|l|} 
Direction \\
\end{tabular} & Signal & $\begin{array}{l}\text { \# of } \\
\text { runs }\end{array}$ & $\begin{array}{c}\text { Run } \\
\#\end{array}$ & $\begin{array}{l}\mathbf{C R} \\
\text { T/F }\end{array}$ & $\begin{array}{c}\mathbf{v}_{\mathbf{f}} \\
\mathrm{mph}\end{array}$ & $\begin{array}{l}\mathbf{v}_{\mathbf{l}} \\
\mathbf{m} / \mathbf{s}\end{array}$ & $\begin{array}{c}\mathbf{y}_{\mathbf{m}} \\
\mathbf{m}\end{array}$ & $\begin{array}{c}\mathbf{y}_{\mathrm{w}} \\
\mathbf{m}\end{array}$ & $\begin{array}{c}T \\
\text { OT/E/L }\end{array}$ \\
\hline 0.5 & 40 & $\leq 0.75$ & Left & No & 5 & 1 & & & & & & \\
\hline 0.5 & $"$ & " & " & " & & $2 \ldots$ & & & & & & \\
\hline 0.5 & 40 & $\leq 0.75$ & Right & No & 5 & & & & & & & \\
\hline 0.5 & 40 & $>0.75$ & Left & No & 5 & & & & & & & \\
\hline 0.5 & 40 & $>0.75$ & Right & No & 5 & & & & & & & \\
\hline 0.5 & 40 & $>0.75$ & Left & On & 1 & & & & & & & \\
\hline 0.5 & 55 & $\leq 0.75$ & Left & No & 5 & & & & & & & \\
\hline 0.5 & 55 & $\leq 0.75$ & Right & No & 5 & & & & & & & \\
\hline 0.5 & 55 & $>0.75$ & Left & No & 5 & & & & & & & \\
\hline 0.5 & 55 & $>0.75$ & Right & No & 5 & & & & & & & \\
\hline 0.5 & 55 & $>0.75$ & Left & On & 1 & & & & & & & \\
\hline 0.5 & 55 & $>0.75$ & Right & On & 1 & & & & & & & \\
\hline 0.5 & 70 & $\leq 0.75$ & Left & No & 5 & & & & & & & \\
\hline 0.5 & 70 & $\leq 0.75$ & Right & No & 5 & & & & & & & \\
\hline 0.5 & 70 & $>0.75$ & Left & No & 5 & & & & & & & \\
\hline 0.5 & 70 & $>0.75$ & Right & No & 5 & & & & & & & \\
\hline 0.5 & 70 & $>0.75$ & Left & On & 1 & & & & & & & \\
\hline 0.5 & 70 & $>0.75$ & Right & On & 1 & & & & & & & \\
\hline 2.0 & 40 & $\leq 0.75$ & Left & No & 5 & & & & & & & \\
\hline 2.0 & 40 & $\leq 0.75$ & Right & No & 5 & & & & & & & \\
\hline 2.0 & 40 & $>0.75$ & Left & No & 5 & & & & & & & \\
\hline 2.0 & 40 & $>0.75$ & Right & No & 5 & & & & & & & \\
\hline 2.0 & 40 & $>0.75$ & Left & On & 1 & & & & & & & \\
\hline 2.0 & 55 & $\leq 0.75$ & Left & No & 5 & & & & & & & \\
\hline 2.0 & 55 & $\leq 0.75$ & Right & No & 5 & & & & & & & \\
\hline 2.0 & 55 & $>0.75$ & Left & No & 5 & & & & & & & \\
\hline 2.0 & 55 & $>0.75$ & Right & $\mathrm{No}$ & 5 & & & & & & & \\
\hline 2.0 & 55 & $>0.75$ & Left & On & 1 & & & & & & & \\
\hline 2.0 & 55 & $>0.75$ & Right & On & 1 & & & & & & & \\
\hline 2.0 & 70 & $\leq 0.75$ & Left & No & 5 & & & & & & & \\
\hline 2.0 & 70 & $\leq 0.75$ & Right & No & 5 & & & & & & & \\
\hline 2.0 & 70 & $>0.75$ & Left & No & 5 & & & & & & & \\
\hline 2.0 & 70 & $>0.75$ & Right & No & 5 & & & & & & & \\
\hline 2.0 & 70 & $>0.75$ & Left & On & 1 & & & & & & & \\
\hline 2.0 & 70 & $>0.75$ & Right & On & 1 & & & & & & & \\
\hline
\end{tabular}

Offset $=$ Offset distance (in meters) of obstacle from outside edge of lane marker 
$\mathrm{CR}=$ System gave correct response $(\mathrm{T})$ or failed to give correct response $(\mathrm{F})$. Refer to subsection 1.1 above to determine correct response.

$\mathrm{v}_{\mathrm{f}}=$ measured vehicle forward speed

$\mathrm{v}_{\mathrm{l}}=$ measured vehicle lateral speed (departure rate)

$\mathrm{y}_{\mathrm{m}}=$ measured lateral distance between the vehicle front tire and the road boundary at the time of warning

$\mathrm{y}_{\mathrm{w}}=$ the desired lateral distance at the time of warning using equation (1)

$\mathrm{T}=$ Timeliness of warning (early, late or on time)

Test Run Summary Results (see section 5.3)

\begin{tabular}{|c|c|c|c|c|c|c|c|c|}
\hline $\begin{array}{c}\text { Combined Results for } \\
\text { all runs }\end{array}$ & $\begin{array}{c}\# \text { of } \\
\text { runs }\end{array}$ & $\begin{array}{c}\# \\
\text { CR }\end{array}$ & $\begin{array}{c}\# \\
\text { OT }\end{array}$ & $\begin{array}{c}\# \\
\text { E }\end{array}$ & $\begin{array}{c}\# \\
\text { L }\end{array}$ & $\begin{array}{c}\text { \% } \\
\text { OT }\end{array}$ & $\begin{array}{c}\text { \% } \\
\text { E }\end{array}$ & $\begin{array}{c}\text { \% } \\
\text { L }\end{array}$ \\
\hline Date/time of test runs & & & & & & & & \\
\hline
\end{tabular}

False Alarm Incident Report

\begin{tabular}{|c|l|}
\hline $\begin{array}{c}\text { Run } \\
\#\end{array}$ & Probable Cause \\
\hline 1 & Example: shadows on road misinterpreted as road edge \\
\hline$\ldots$ & $\ldots$ \\
\hline
\end{tabular}




\subsection{Obstacle on Shoulder on Curved Road}

The Obstacle on Shoulder on Curved Road test assesses the warning system's ability to detect an obstacle on the shoulder ahead of the vehicle and to adjust the warning location on a curved road departure.

The figure below illustrates the test scenario. The left-side collision avoidance test uses a right turn curve.

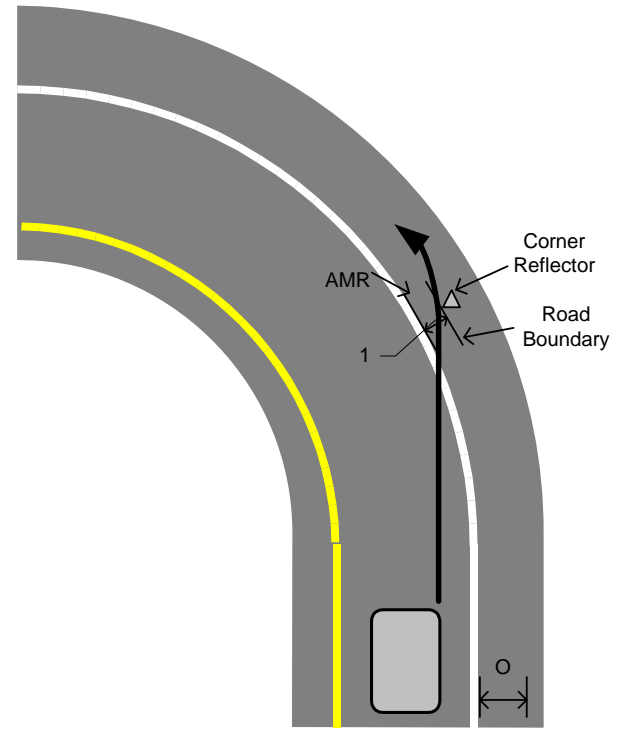

A

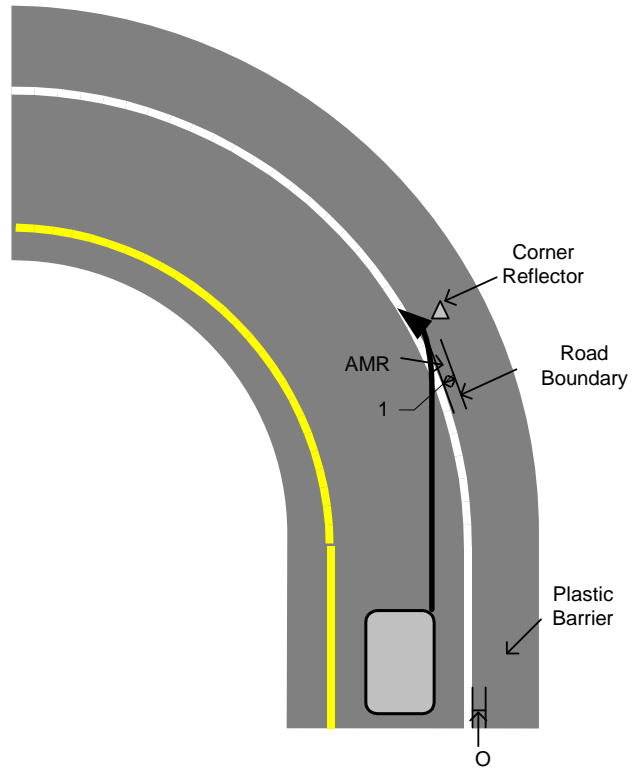

B

Figure 30. Obstacle on Shoulder on Curved Road Test, Vehicle trajectory

\subsubsection{Events and System Response}

The following table defines the events for this test and the correct system response. See the figure above to locate the event (e.g., Id A1 is label 1 in figure A).

\begin{tabular}{|c|l|l|}
\hline Id & \multicolumn{1}{|c|}{ Event } & \multicolumn{1}{c|}{ Correct Response } \\
\hline A1 & Lateral collision & 1. Issue lateral collision warning \\
\hline B1 & Lateral collision & 1 . Same as A1 \\
\hline
\end{tabular}

\subsubsection{Test Conditions}

The conditions for this test include the following. 


\subsubsection{Road Geometry}

The curve radius for this test should be appropriate for the low speed, medium speed and high-speed conditions. The following equation calculates the curve radius for each speed condition using lateral acceleration limits $\left(a_{\text {lat }}\right)$ from section 2.3.5.

$r=\frac{v^{2}}{a_{\text {lat }}}$

For the test, the curve radius falls within a range of radii by using the lateral acceleration limits for sensitivities 2 and 4.

\begin{tabular}{|l|l|}
\hline Road Geometry & Dimension \\
\hline Low speed curve, $64 \mathrm{~km} / \mathrm{h}(40 \mathrm{mph})$ & $91 \mathrm{~m}-136 \mathrm{~m}$ \\
\hline Medium speed curve, $89 \mathrm{~km} / \mathrm{h}(55 \mathrm{mph})$ & $172 \mathrm{~m}-257 \mathrm{~m}$ \\
\hline Low speed curve, $113 \mathrm{~km} / \mathrm{h}(70 \mathrm{mph})$ & $278 \mathrm{~m}-417 \mathrm{~m}$ \\
\hline
\end{tabular}

Appendix A, Methods for Determining Road Dimensions and Geometry, contains the recommended method to verify the road geometry.

\subsubsection{Maximum Available Maneuver Room}

In this test, obstacle offset determines the AMR. However, the AMR is limited to a maximum value to prevent large variations warning location caused by large variations shoulder width.

\begin{tabular}{|l|l|}
\hline Available Maneuver Room & Comment \\
\hline $1 \mathrm{~m}(39$ in $)$ & Maximum AMR \\
\hline
\end{tabular}

\subsubsection{Speed}

The vehicle speeds during the tests cover the warning system operating range.

\begin{tabular}{|l|l|l|}
\hline Low Speed & Medium Speed & High Speed \\
\hline $64 \mathrm{~km} / \mathrm{h}(40 \mathrm{mph})$ & $89 \mathrm{~km} / \mathrm{h}(55 \mathrm{mph})$ & $113 \mathrm{~km} / \mathrm{h}(70 \mathrm{mph})$ \\
\hline
\end{tabular}

\subsubsection{Obstacle}

For safety purposes, this test uses a plastic, traffic barrel (see Figure 28).

\subsubsection{Obstacle Offset}

The tests involve placing the obstacles at two offsets measured from the outside edge of the lane marker. The small offset is one half the maximum AMR and the large offset is twice the AMR. The test operator updates these values if the default minimum or maximum AMR changes.

\begin{tabular}{|l|l|}
\hline Barrier Offset Name & \multicolumn{1}{c|}{ Dimension } \\
\hline Small Offset & $0.5 \mathrm{~m}(19.5 \mathrm{in})$ \\
\hline Large Offset & $2 \mathrm{~m}(79 \mathrm{in})$ \\
\hline
\end{tabular}




\subsubsection{Measurements}

Measurements determine whether a warning was given, the desired location of a warning given the conditions and the amount of time the warning gives driver.

\subsubsection{Time of Warning}

See section 2.3.9.

\subsubsection{Longitudinal Speed}

See section 2.2.2.

\subsubsection{Distance to Road Edge}

See section 2.2.7. For this test, the base of the obstacle defines the road edge. If the obstacle is not adjacent to the front wheel at the time of warning and a direct measurement is not possible, then the evaluator calculates the distance to the road edge using the distance to the lane marker plus the obstacle offset (see Figure 29). To take into account the departure angle ( $\theta$ requires departure rate, see section 2.2.9), use the following equation:

$y_{w}=l+h=l+\frac{o}{\cos \theta}$

Where:

$y_{w}=$ the lateral distance between the vehicle front tire and the road boundary

$l=$ the measured lateral distance to the lane marker

$o=$ the known offset of the obstacle

$\theta=$ the departure angle

\subsubsection{Distance to Curve Entry Point}

See section 2.2.10.

\subsubsection{Test Procedure}

The general procedure for the test repeats a series of road departure trajectories depicted in Figure 30.

\subsubsection{Pre-Test Procedures}

The procedures before the test are:

1. Ensure road geometry meets the test condition. The operator measures the road geometry as per Appendix A if required.

2. Ensure the lane marker characteristics meet the test conditions. The operator measures the characteristics if it is unknown (see sections 2.1.1-2.1.12).

3. Check speedometer accuracy (see section 2.2.2).

4. Verify IMS camera calibration according to recommendations in [5]. Repeat camera calibration if camera has moved.

5. Ensure microphone detects warning system alerts and driver speech.

6. Check Ensure recording system is working properly. 
7. Ensure the GPS has sufficient satellites and operates properly.

8. Assemble the barrels and position for tests (use large offset for first test).

9. Verify road boundary for test (i.e., outer lane marker plus AMR) and practice vehicle trajectories (speeds, departure rate, etc.) until accomplished safely and reliably.

\subsubsection{Test Procedures}

The procedures during the test are:

1. Achieve speed. The driver brings the vehicle to the prescribed speed (low speed curve is $64 \mathrm{~km} / \mathrm{h}(40 \mathrm{mph}))$ and sets the cruise control at the speed.

2. Straighten and center vehicle before entering curve. The driver centers the vehicle with the lane markers as a visual guide. The vehicle remains in the center until the start of the curve.

3. Maintain straight trajectory into the curve. As the vehicle enters the curve, the driver uses the obstacle as a guide to maintain a straight trajectory toward the collision.

4. Collide with obstacle. The driver continues straight until a warning takes place or the driver aborts before collision.

5. Repeat steps 1-4 for:

a. Five right-side collisions.

b. Five left-side collisions.

6. Repeat steps $1-5$ for the collision on the middle speed curve.

7. Repeat steps $1-5$ for the collision on the high-speed curve.

8. Repeat steps $1-7$ with the obstacles at the large offset.

\subsubsection{Post-Test Procedures}

The procedures after the test are:

1. Verify IMS camera orientation has not changed. Repeat tests if changed.

\subsubsection{Evaluation Methods}

Use the following methods to evaluate warning system performance.

\subsubsection{Rate System Response}

Examine the video and audio data and rate each warning scenario.

The rating is a true positive if the system responds with a warning before the road boundary is crossed.

The rating is a false negative if the system does not respond with a warning before the road boundary is crossed. 


\subsubsection{Desired Location of Warning}

For each test run, the evaluator determines the desired location of the warning. The desired location varies with the forward speed and curve radii.

1. Locate the time of warning in the video data (for example, by locating video frame where alert is first displayed).

2. Measure lateral position and lateral velocity to the road edge at time of warning.

3. Use equation (4) in section 5.1.2 to determine the desired location for a warning on a curve.

\subsubsection{Timeliness of Warning}

The evaluator determines the timeliness of each warning (early, late or on time) and compiles statistics according to section 5.3.

\subsubsection{Reporting}

The evaluator completes the following tables from the data in each test.

\begin{tabular}{|l|l|}
\hline Name of Test & $\begin{array}{l}\text { Collision with Obstacle on Curved } \\
\text { Road }\end{array}$ \\
\hline Date of Test & \\
\hline Warning System ID & Name and Version Number \\
\hline
\end{tabular}

Test Run Results

\begin{tabular}{|l|l|l|l|l|l|l|l|l|l|l|}
\hline \multicolumn{9}{|c|}{ Factors } & \multicolumn{7}{c|}{ Results } \\
\hline $\begin{array}{c}\text { Offset } \\
\mathbf{m}\end{array}$ & Curve & $\begin{array}{c}\text { Speed } \\
\mathbf{m p h}\end{array}$ & Direction & $\begin{array}{c}\text { \# of } \\
\text { runs }\end{array}$ & $\begin{array}{c}\text { Run } \\
\#\end{array}$ & $\begin{array}{c}\mathbf{C R} \\
\mathbf{T} / \mathbf{F}\end{array}$ & $\begin{array}{c}\mathbf{v}_{\mathbf{f}} \\
\mathbf{m p h}\end{array}$ & $\begin{array}{c}\mathbf{y}_{\mathbf{m}} \\
\mathbf{m}\end{array}$ & $\begin{array}{c}\mathbf{x}_{\mathbf{w}} \\
\mathbf{m}\end{array}$ & $\begin{array}{c}\mathbf{T} \\
\mathbf{\text { OT/E/L }}\end{array}$ \\
\hline 0.075 & LS & 40 & Left & 5 & 1 & & & & & \\
\hline$"$ & $"$ & $"$ & $"$ & & $2 \ldots$ & & & & & \\
\hline 0.075 & LS & 40 & Right & 5 & & & & & & \\
\hline 0.075 & MS & 55 & Left & 5 & & & & & & \\
\hline 0.075 & MS & 55 & Right & 5 & & & & & & \\
\hline 0.075 & HS & 70 & Left & 5 & & & & & & \\
\hline 0.075 & HS & 70 & Right & 5 & & & & & & \\
\hline 2.0 & LS & 40 & Left & 5 & & & & & & \\
\hline 2.0 & LS & 40 & Right & 5 & & & & & & \\
\hline 2.0 & MS & 55 & Left & 5 & & & & & & \\
\hline 2.0 & MS & 55 & Right & 5 & & & & & & \\
\hline 2.0 & HS & 70 & Left & 5 & & & & & & \\
\hline 2.0 & HS & 70 & Right & 5 & & & & & & \\
\hline
\end{tabular}

Offset $=$ Offset distance (in meters) of obstacle from outside edge of lane marker

Curve $=$ Low speed curve, medium speed curve and high speed curve

$\mathrm{CR}=$ System gave correct response $(\mathrm{T})$ or failed to give correct response $(\mathrm{F})$. Refer to subsection 1.1 above to determine correct response.

$\mathrm{v}_{\mathrm{f}}=$ vehicle forward speed 
$\mathrm{y}_{\mathrm{m}}=$ the measured lateral distance between the vehicle front tire and the road boundary at the time of warning

$\mathrm{x}_{\mathrm{W}}=$ the desired distance to curve entry point at the time of warning using equation (4)

$\mathrm{T}=$ Timeliness of warning (early, late or on time)

Test Run Summary Results (see section 5.3)

\begin{tabular}{|c|c|c|c|c|c|c|c|c|}
\hline $\begin{array}{c}\text { Combined Results for } \\
\text { all runs }\end{array}$ & $\begin{array}{c}\# \text { of } \\
\text { runs }\end{array}$ & $\begin{array}{c}\# \\
\text { CR }\end{array}$ & $\begin{array}{c}\# \\
\text { OT }\end{array}$ & $\begin{array}{c}\# \\
\text { E }\end{array}$ & $\begin{array}{c}\# \\
\text { L }\end{array}$ & $\begin{array}{c}\text { \% } \\
\text { OT }\end{array}$ & $\begin{array}{c}\text { \% } \\
\text { E }\end{array}$ & $\begin{array}{c}\text { L } \\
\text { L }\end{array}$ \\
\hline Date/time of test runs & & & & & & & & \\
\hline
\end{tabular}

False Alarm Incident Report

\begin{tabular}{|c|l|}
\hline $\begin{array}{c}\text { Run } \\
\#\end{array}$ & Probable Cause \\
\hline 1 & Example: shadows on road misinterpreted as road edge \\
\hline$\ldots$ & $\ldots$ \\
\hline
\end{tabular}




\section{Tests for Departures on Curves at Excessive Speed}

The tests for Departures on Curves at Excessive Speed evaluate the warning system's ability to detect whether the vehicle is traveling too fast for an approaching curve and to warn the driver in time to slow the vehicle to a safe speed. Since the curve may be beyond visual limits, curve-speed warnings rely on a map to obtain the curvature of upcoming road segments and a positioning system (e.g., Global Positioning System) to provide the vehicle location relative to the curve. The system estimates the amount of deceleration required to slow the vehicle to a safe speed given the distance to the curve. The safe speed takes into account the radius of the curve and other available data such as temperature and precipitation (e.g., windshield wiper on or off), which provides an indirect measure of road friction. The speeds and curve radii are consistent with a rural road scenario where this type of crash most often occurs.

The curve-branch test examines the system's ability to correctly determine whether the vehicle is taking the curve or straight branch when a road network splits. Curve-speed warnings for the curve-branch of the road are a nuisance when the vehicle is heading toward the straight branch of the road. 


\subsection{Excessive Speed on Curved Road}

The Excessive Speed on Curved Road test examines the performance of the curve speed warning system for the scenario where a vehicle is traveling too fast for an upcoming curve in the road. This scenario falls under the "negotiating a curve on undivided nonfreeway with two lanes at non-junction" scenario identified in the NHTSA test scenario report as one of the major settings for road departure crashes [3]. This test assumes the warning system uses GPS and a digital map to determine the distance to approaching curves and the radius of the curve.

The figure below illustrates the test scenario.

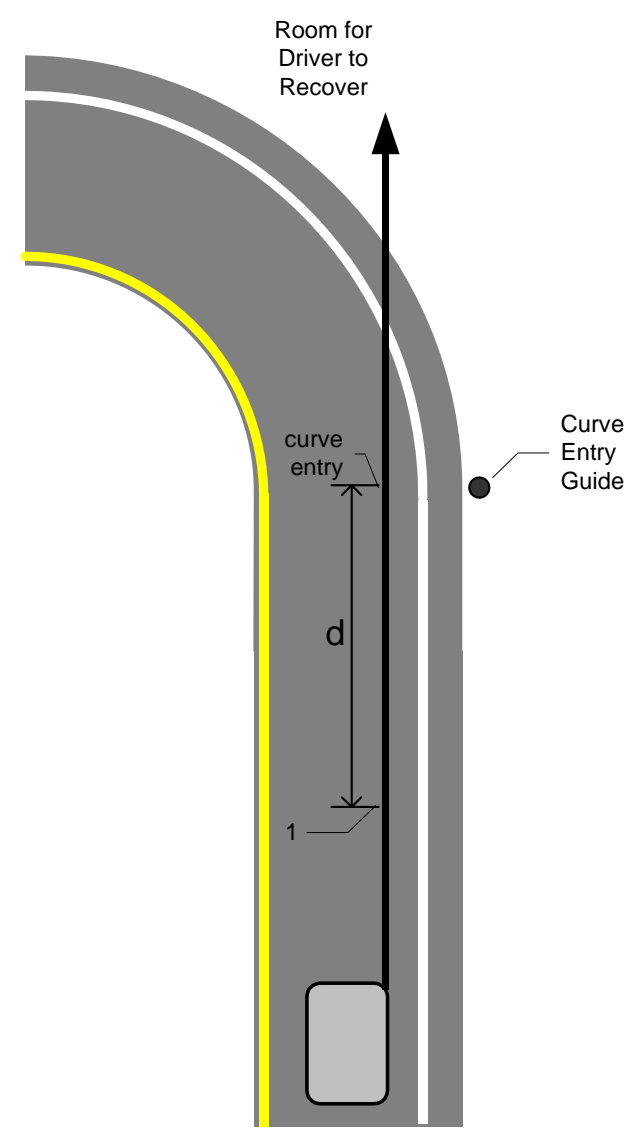

Figure 31. Excessive Speed on Curved Road Test, Vehicle Trajectory

\subsubsection{Events and System Response}

The following table defines the events for this test and the correct warning signal the system shall provide. The primary event is the vehicle reaching a distance from a curve 
where the current speed is too fast for the driver to safely negotiate the curve. See the figure above to locate the event.

\begin{tabular}{|c|c|c|}
\hline Id & Event & Correct Response \\
\hline 1 & Excessive speed for curve & 1 . Issue excessive speed for curve warning \\
\hline
\end{tabular}

\subsubsection{Test Conditions}

The conditions for this test include the following.

\subsubsection{Road Geometry}

The test examines two types of curves suggested in [3]. A range of curve radii provides flexibility in selecting suitable test sites.

\begin{tabular}{|l|l|}
\hline Road Type & Dimension \\
\hline Non-freeway & $50 \mathrm{~m}-100 \mathrm{~m}$ \\
\hline Freeway & $200 \mathrm{~m}-350 \mathrm{~m}$ \\
\hline
\end{tabular}

Appendix A, Methods for Determining Road Dimensions and Geometry, contains the recommended method to verify the road geometry.

\subsubsection{Temperature}

The temperature condition for the test falls within the following ranges:

\begin{tabular}{|l|l|}
\hline Condition & Temperature \\
\hline Cold & $0^{\circ} \mathrm{C}-3^{\circ} \mathrm{C}\left(32^{\circ} \mathrm{F}-38^{\circ} \mathrm{F}\right)$ \\
\hline Warm & $4^{\circ} \mathrm{C}-7^{\circ} \mathrm{C}\left(39^{\circ} \mathrm{F}-45^{\circ} \mathrm{F}\right)$ \\
\hline
\end{tabular}

Artificial means to achieve the temperature at the sensor are acceptable (e.g., ice).

\subsubsection{Precipitation}

The precipitation during the test is either dry or wet. The warning system determines precipitation from windshield wiper status.

\begin{tabular}{|l|l|}
\hline Condition & Windshield Wiper \\
\hline Dry & Off \\
\hline Wet & On \\
\hline
\end{tabular}

\subsubsection{Speed}

The test requires the driver to approach the curve at a speed in excess of the safe speed for the curve. Use equation (6) to compute the safe speed for the curve. Use $3 \mathrm{~m} / \mathrm{s}^{2}(0.3$ g) lateral acceleration limit (sensitivity $=3$ ) for warm, dry condition. Use $1 \mathrm{~m} / \mathrm{s}^{2}(0.1 \mathrm{~g})$ for cold, wet condition ([12] page 10.27). The following table provides an example of the speeds to use for a test on two radii chosen for freeway and non-freeway roads. 


\begin{tabular}{|l|l|l|c|c|c|}
\hline Road Type & $\begin{array}{c}\text { Curve } \\
\text { Radius }\end{array}$ & Condition & Safe speed & $\begin{array}{c}\text { Over- } \\
\text { speed 1 } \\
(\mathbf{1 1 5} \%)\end{array}$ & $\begin{array}{c}\text { Over- } \\
\text { speed 2 } \\
(\mathbf{1 3 0} \%)\end{array}$ \\
\hline Freeway & \multirow{2}{*}{$200 \mathrm{~m}$} & Warm, dry & $\begin{array}{c}87 \mathrm{~km} / \mathrm{h} \\
(54 \mathrm{mph})\end{array}$ & $\begin{array}{c}100 \mathrm{~km} / \mathrm{h} \\
(62 \mathrm{mph})\end{array}$ & $\begin{array}{c}113 \mathrm{~km} / \mathrm{h} \\
(70 \mathrm{mph})\end{array}$ \\
\cline { 3 - 6 } & & Cold, wet & $\begin{array}{c}50 \mathrm{~km} / \mathrm{h} \\
(31 \mathrm{mph})\end{array}$ & $\begin{array}{c}58 \mathrm{~km} / \mathrm{h} \\
(36 \mathrm{mph})\end{array}$ & $\begin{array}{c}68 \mathrm{~km} / \mathrm{h} \\
(41 \mathrm{mph})\end{array}$ \\
\hline $\begin{array}{l}\text { Non- } \\
\text { Freeway }\end{array}$ & \multirow{2}{*}{$75 \mathrm{~m}$} & Warm, dry & $\begin{array}{c}53 \mathrm{~km} / \mathrm{h} \\
(33 \mathrm{mph})\end{array}$ & $\begin{array}{c}61 \mathrm{~km} / \mathrm{h} \\
(38 \mathrm{mph})\end{array}$ & $\begin{array}{c}33 \mathrm{~km} / \mathrm{h} \\
(38 \mathrm{mph})\end{array}$ \\
\cline { 3 - 6 } & & Cold, wet & $\begin{array}{c}31 \mathrm{~km} / \mathrm{h} \\
(19 \mathrm{mph})\end{array}$ & $\begin{array}{c}35 \mathrm{~km} / \mathrm{h} \\
(22 \mathrm{mph})\end{array}$ & $\begin{array}{c}40 \mathrm{~km} / \mathrm{h} \\
(25 \mathrm{mph})\end{array}$ \\
\hline
\end{tabular}

\subsubsection{Measurements}

Measurements collected during the test support the following:

1. Determine whether a warning was given

2. Determine the desired location of a warning given the conditions

3. Determine the amount of time the warning gives driver

\subsubsection{Time of Warning}

See section 2.3.9.

\subsubsection{Longitudinal Speed}

See section 2.2.2.

\subsubsection{Distance to Curve Entry Point}

See section 2.2.10.

\subsubsection{Test Procedure}

The general procedure for the test repeats a series of road departure trajectories depicted in Figure 31.

\subsubsection{Pre-Test Procedures}

The procedures before the test are:

1. Ensure road geometry meets the test condition and measure the geometry as per Appendix A if required.

2. Ensure the lane marker characteristics meet the test conditions. The operator measures the characteristics if it is unknown (see sections 2.1.1-2.1.12).

3. Check speedometer accuracy (see section 2.2.2).

4. Verify IMS camera calibration according to recommendations in [5]. Repeat camera calibration if camera has moved.

5. Ensure microphone detects warning system alerts and driver speech. 
6. Ensure recording system is working properly.

7. Ensure the GPS has sufficient satellites and is operating properly.

8. Establish proper initial sensor conditions (initial test is a warm, dry, non-freeway scenario).

9. Verify curve entry point for test (i.e., outer lane marker plus AMR) and practice vehicle trajectories (speeds, decelerations, etc.) until accomplished safely and reliably. Ensure recovery area exists in case driver is not able to keep vehicle in lane through curve.

\subsubsection{Test Procedures}

The procedures during the test are:

1. Achieve temperature. Set the system's temperature sensor too output prescribed condition (warm or cold).

2. Achieve precipitation condition. Set the windshield wiper to the prescribed condition $($ dry $=$ off, wet $=$ on $)$.

3. Achieve speed. The driver brings the vehicle to the over-speed condition.

4. Maintain vehicle centered in lane until past curve entry point. Vehicle may depart out of lane into recovery area if traveling too fast.

5. Document the warning type and result.

6. Repeat steps 1- 5 five times.

7. Repeat steps $1-6$ for over-speed 2 ( $130 \%$ of maximum)

8. Repeat steps $1-7$ for cold, wet condition

9. Repeat steps $1-8$ for freeway

\subsubsection{Post-Test Procedures}

The procedures after the test are:

1. Verify IMS camera orientation has not changed. Repeat tests if changed.

\subsubsection{Evaluation Methods}

Use the following methods to evaluate warning system performance.

\subsubsection{Rate System Response}

Examine the video and audio data and rate each warning scenario.

The rating is a true positive if the system responds with a warning before the vehicle has completed travel through the curve.

The rating is a false negative if the system does not respond with a warning before the vehicle exits the curve.

\subsubsection{Desired Location of Warning}

For each test run, the evaluator determines the desired location of the warning. 
1. Locate the time of warning in the video data (for example, by locating video frame where alert is first displayed).

2. Measure distance to curve entry at time of warning.

3. Use equation (7) in section 5.2 to determine the desired location for a warning when traveling too fast for an approaching curve.

\subsubsection{Timeliness of Warning}

For each test run, the evaluator determines whether the warning is early, late or on time using steps described in section 5.2. The results of the timeliness evaluations are accumulated according to section 5.3.

\subsubsection{Reporting}

The evaluator completes the following tables from the data in each test.

\begin{tabular}{|l|l|}
\hline Name of Test & Curve Speed Warning Test \\
\hline Date of Test & \\
\hline Warning System ID & Name and Version Number \\
\hline
\end{tabular}

Test Run Results

\begin{tabular}{|l|l|l|l|l|l|l|l|l|}
\hline \multicolumn{3}{|c|}{ Factors } & \multicolumn{6}{c|}{ Results } \\
\hline Condition & $\begin{array}{c}\text { Speed } \\
\mathbf{m p h}\end{array}$ & $\begin{array}{c}\text { \# of } \\
\text { runs }\end{array}$ & $\begin{array}{c}\text { Run } \\
\#\end{array}$ & $\begin{array}{c}\text { CR } \\
\mathbf{T} / \mathbf{F}\end{array}$ & $\begin{array}{c}\mathbf{v}_{\mathbf{f}} \\
\mathbf{m p h}\end{array}$ & $\begin{array}{c}\mathbf{x}_{\mathbf{m}} \\
\mathbf{m}\end{array}$ & $\begin{array}{c}\mathbf{x}_{\mathbf{w}} \\
\mathbf{m}\end{array}$ & $\begin{array}{c}\mathbf{T} \\
\text { OT/E/ } \\
\mathbf{L}\end{array}$ \\
\hline Warm/Dry & 67 & 10 & 1 & & & & & \\
\hline$"$ & $"$ & $"$ & $2 \ldots$ & & & & & \\
\hline Cold/Wet & 52 & 10 & 1 & & & & & \\
\hline
\end{tabular}

$\mathrm{CR}=$ System gave correct response $(\mathrm{T})$ or failed to give correct response $(\mathrm{F})$. Refer to subsection 1.1 above to determine correct response.

$\mathrm{v}_{\mathrm{f}}=$ measured vehicle forward speed

$\mathrm{x}_{\mathrm{m}}=$ The measured distance to the curve at the time of warning

$\mathrm{x}_{\mathrm{W}}=$ the desired distance to the curve at the time of warning using equation (7)

$\mathrm{T}=$ Timeliness of warning (early, late or on time)

Test Run Summary Results (see section 5.3)

\begin{tabular}{|c|c|c|c|c|c|c|c|c|}
\hline $\begin{array}{c}\text { Combined Results for } \\
\text { all runs }\end{array}$ & $\begin{array}{c}\# \text { of } \\
\text { runs }\end{array}$ & $\begin{array}{c}\# \\
\text { CR }\end{array}$ & $\begin{array}{c}\# \\
\text { OT }\end{array}$ & $\begin{array}{l}\# \\
\text { E }\end{array}$ & $\begin{array}{c}\# \\
\text { L }\end{array}$ & $\begin{array}{c}\text { O } \\
\text { OT }\end{array}$ & $\begin{array}{c}\text { \% } \\
\text { E }\end{array}$ & $\begin{array}{c}\text { L } \\
\text { L }\end{array}$ \\
\hline Date/time of test runs & & & & & & & & \\
\hline
\end{tabular}

False Alarm Incident Report

\begin{tabular}{|c|l|}
\hline $\begin{array}{c}\text { Run } \\
\#\end{array}$ & Probable Cause \\
\hline 1 & Example: shadows on road misinterpreted as road edge \\
\hline 2 & Example: longitudinal road joint misinterpreted as road edge \\
\hline$\ldots$ & $\ldots$ \\
\hline
\end{tabular}




\subsection{Curve-Branch Detection}

The Curve-Branch Detection test examines whether the curve-speed warning system accurately predicts whether a vehicle is about to travel on a straight or curved branch of a road that splits. This test assumes the warning system uses GPS and a digital map to determine the distance to approaching curves and the radius of the curve. The scenario for this test requires a two-lane road that splits into a straight road and a curved road.

The curve-branch requires sufficient radius to generate a warning at a reasonable approach speed. For test purposes, the rural road curve used in the curve-speed warning test may be adapted for the curve-branch test.

During the curve-branch test, the system issues a warning if the vehicle is in the lane headed toward the curve at a high speed (see B in figure below). Conversely, the system does not issue a warning if the vehicle is in the lane headed straight (see A in figure below). The test verifies correct operation in both lanes. The driver uses the procedure in section 9.1 for the test in the curve lane and the procedure below for the straight lane.

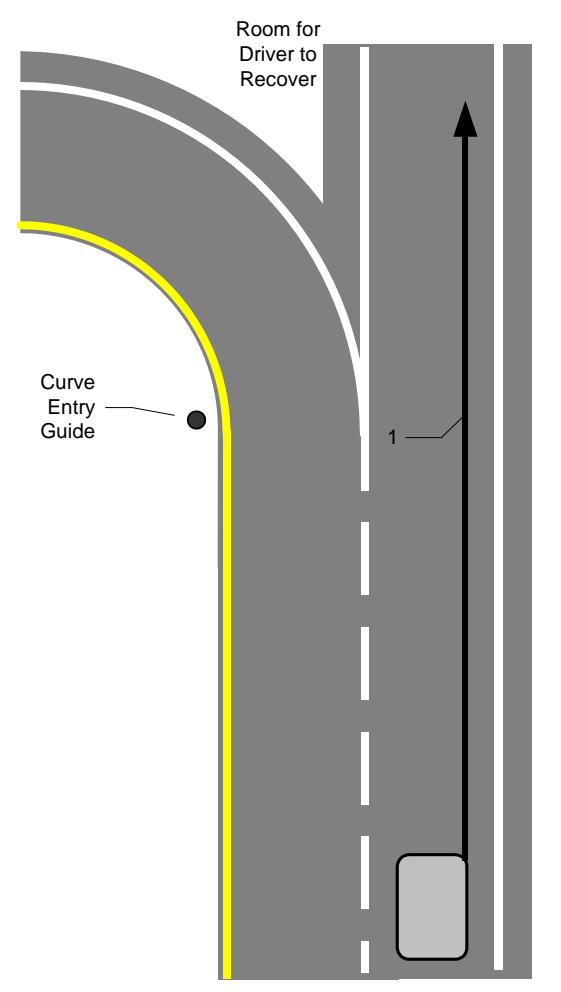

A

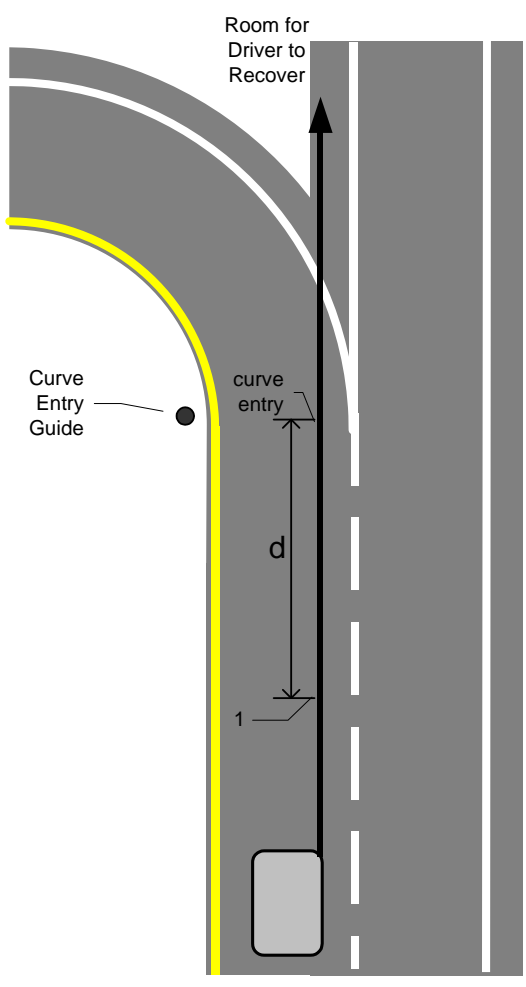

B

Figure 32. Curve-Branch Test, Vehicle trajectory 


\subsubsection{Events and System Response}

The following table defines the events for this test and the correct warning signal the system shall provide. The primary event is the vehicle reaching a distance from a curve where the current speed is too fast for the driver to safely negotiate the curve. See the figure above to locate the event.

\begin{tabular}{|c|l|l|}
\hline Id & \multicolumn{1}{|c|}{ Event } & \multicolumn{1}{c|}{ Correct Response } \\
\hline A1 & Reached Curve & 1. No warning should be given \\
\hline
\end{tabular}

\subsubsection{Test Conditions}

The conditions for this test include the following.

\subsubsection{Road Geometry}

The road geometry for this test consists of a straight lane adjacent to a curved lane. See section 9.1.2.1 for the curved lane radii. It is sufficient to test the freeway curve only. The straight lane has the following geometry:

\begin{tabular}{|l|l|}
\hline Road Geometry & Dimension \\
\hline Straight lane (radius) & $>1000 \mathrm{~m}$ \\
\hline
\end{tabular}

Appendix A, Methods for Determining Road Dimensions and Geometry, contains the recommended method to verify the road geometry.

\subsubsection{Temperature}

Use same temperature conditions as in section 9.1.2.2.

\subsubsection{Precipitation}

Use same precipitation conditions as in section 9.1.2.3.

\subsubsection{Speed}

The speeds in the straight lane are the same as those for the curved lane. See section 9.1.2.4.

\subsubsection{Measurements}

Measurements determine whether a warning was given and whether the test speed was within limits.

\subsubsection{Time of Warning}

See section 2.3.9.

\subsubsection{Longitudinal Speed}

See section 2.2.2. 


\subsubsection{Test Procedure}

The general procedure for the test repeats a series of road departure trajectories depicted in Figure 32. The driver conducts this test after the curve over-speed test.

\subsubsection{Pre-Test Procedures}

The procedures before the test are:

1. Ensure road geometry meets the test condition and measure the geometry as per Appendix A if required.

2. Ensure the lane marker characteristics meet the test conditions. The operator measures the characteristics if it is unknown (see sections 2.1.1-2.1.12).

3. Check speedometer accuracy (see section 2.2.2).

4. Verify IMS camera calibration according to recommendations in [5]. Repeat camera calibration if camera has moved.

5. Ensure microphone detects warning system alerts and driver speech.

6. Ensure recording system is working properly.

7. Ensure the GPS has sufficient satellites and is operating properly.

8. Establish proper initial sensor conditions (initial test is a warm, dry, non-freeway scenario)

\subsubsection{Test Procedures}

The procedures during the test are:

1. Achieve temperature. Set the system's temperature sensor to prescribed condition.

2. Achieve precipitation condition. Set the windshield wiper to the prescribed condition $($ dry $=$ off, wet $=$ on $)$.

3. Achieve speed. The driver brings the vehicle to the over-speed condition.

4. Maintain vehicle centered in lane until past the split.

5. Document the warning type and result.

a. Repeat steps 1-5 five times.

6. Repeat steps $1-5$ for cold, wet condition

\subsubsection{Post-Test Procedures}

The procedures after the test are:

1. Verify IMS camera orientation has not changed. Repeat tests if changed.

\subsubsection{Evaluation Methods}

Use the following methods to evaluate warning system performance.

\subsubsection{Rate System Response}

Examine the video and audio data and rate each warning scenario.

The rating is a false positive if the system responds with a warning while the vehicle approaches the split while inside the straight lane markers. 


\subsubsection{Reporting}

The evaluator completes the following tables from the data in each test.

\begin{tabular}{|l|l|}
\hline Name of Test & Curve Speed Warning Test \\
\hline Date of Test & \\
\hline Warning System ID & Name and Version Number \\
\hline
\end{tabular}

Test Run Results

\begin{tabular}{|c|l|c|c|c|c|c|}
\hline \multicolumn{3}{|c|}{ Factors } & \multicolumn{3}{|c|}{ Results } \\
\hline Condition & $\begin{array}{c}\text { Speed } \\
\text { mph }\end{array}$ & $\begin{array}{c}\text { \# of } \\
\text { runs }\end{array}$ & $\begin{array}{c}\text { Run } \\
\#\end{array}$ & $\begin{array}{c}\text { CR } \\
\text { T/F }\end{array}$ & $\begin{array}{c}\mathbf{v}_{\mathbf{f}} \\
\mathbf{m p h}\end{array}$ & Possible causes of false alarm \\
\hline Warm/Dry & 67 & 10 & 1 & & & \\
\hline$"$ & $"$ & \multicolumn{1}{|c|}{$"$} & $2 \ldots$ & & & \\
\hline
\end{tabular}

$\mathrm{CR}=$ System gave correct response ( $\mathrm{T}$ if no warning) or failed to give correct response ( $\mathrm{F}$ if false alarm).

$\mathrm{v}_{\mathrm{f}}=$ measured vehicle forward speed 


\section{References}

[1] Request for Applications for Cooperative Agreement DTFH61-01-X-00053, A Intelligent Vehicle Initiative Road Departure Crash Warning Field Operational Test, April 11, 2001, http://www.eps.gov/EPSData/DOT/Synopses/30/DTFH61-01-X00053/DTFH61-01-X-00053.rtf

[2] "Run-Off-Road Collision Avoidance Using IVHS Countermeasures, Final Report", Contract No. DTNH22-93-C-07023, USDOT National Highway Traffic Safety Administration, Office of Collision Avoidance Research, December 141999.

[3] Wassim Najm, Jonathan Koopmann, Linda Boyle, Dave Smith, "Development of Test Scenarios for Off-Roadway Crash Countermeasures on Crash Statistics", May 2001, Report Number DOT-VNTSC-NHTSA-01-02.

[4] "Standardization Working Draft for PWI14.8 - Lane Departure Warning Systems," International Standards Organization Technical Committee 204, Working Group 14, October 23, 2000. This can be obtained from Steven Shladover, sess@uclink4.berkeley.edu

[5] Sandor Szabo, Richard Norcross, "An Independent Measurement System for Performance Evaluation of Road Departure Crash Warning Systems," August 14, 2005. Can be obtained from Sandor Szabo, sandor.szabo@nist.gov.

[6] "Vehicle Dynamics Terminology," SAE J670e, July 1976.

[7] [4] Deal, F.C., Men, H., Phamdo, N., "Single Vehicle Roadway Departure Prevention Program - Public Curve Speed Warning (CSW) and Lateral Drift Warning (LDW) Algorithms Design and Performance", Draft report prepared by Johns Hopkins Applied Physics Laboratory, April 2004.

[8] August Burgett, "DOT's Approach to ITS Safety Evaluations" invited papers to ITS America Safety Evaluation Workshop, May 1, 1995.

[9] August Burgett, Kirsten Gunderson, "Crash Prevention Boundary for Road Departure Crashes---Derivation,” Research Note DOT HS 809 399, September 2001.

[10] Bruce Wilson, August Burgett, "Crash Prevention Boundaries for Road Departure," In Ninth World Congress on Intelligent Transportation Systems, Chicago, IL, October 2002.

[11] A Policy on Geometric Design of Highways and Streets, American Association of State Highway and Transportation Officials, 1994. 
[12] R. Lamm, B. Psarianos, T.Mailaender, Highway Design and Traffic Safety Engineering Handbook, McGraw-Hill NY, 1999.

[13] ASTM D 6628 - 01, "Standard Specification for Color of Pavement Marking Materials"

[14] European Standard EN 1436:1997/prA1:2002 "Road marking materials - Road Marking Performance for Road Users" 


\section{Appendix A Methods for Determining Road Dimensions and Geometry}

This section contains recommended methods to verify that road segments satisfy the geometry specifications prescribed for the test.

\section{A.1 Lane Marker Dimensions}

Use a tape measure to verify lane marker dimensions before the test. Alternatively, use the IMS to measure lane marker dimensions.

\section{A.2 Lane Width}

Use a tape measure to verify lane width before the test. Alternatively, use the IMS to measure lane width.
A.3 Curve Radius
$r=\frac{v}{\theta^{\prime}}$,
Where:
$r=$ radius of curve $(\mathrm{m})$
$v=$ vehicle forward speed $(\mathrm{m} / \mathrm{s})$
$\theta^{\prime}=$ the yaw rate (radians $\left./ \mathrm{s}\right)$.

Calculate the curve radius of the road segment over the entire road segment or over the portion of the road segmented or at the point of road departure.

Calculate the curve radius from vehicle speed and a yaw rate sensor (gyro) using the following formula (derived from arc length formula):

If a GPS is used, calculate the curvature using a three points measured on the curve using the Independent Measurement System to visually measure road edge points. 


\section{Appendix B Methods for Determining Lane Marker Characteristics}

This appendix provides standards for measuring and attaining color and reflectivity properties of horizontal pavement markings to ensure that lane markings provide appropriate conspicuity for the warning system sensor. The performance of pavement markings is determined by the coefficient of retroreflected luminance, $R_{L}$, the coefficient of retroreflected luminance for wet roadways, $R_{L-W e t}$, the coefficient of retroreflected luminance under continuous wetting, $R_{L-R a i n}$, or the luminance coefficient under diffuse illumination, $\mathrm{Q}_{\mathrm{d}}$. Under the same conditions of headlight illumination and driver's viewing, larger values of these coefficients correspond to higher levels of visual performance at corresponding geometry. Reapply the lane markings when they measure outside of the specified value.

The following standard apply for lane-marker reflectance specifications:

ASTM D 6628 - 01, "Standard Specification for Color of Pavement Marking Materials", ASTM D 6359 - 99, "Standard Specification for Minimum Retroreflectance of Newly Applied Pavement Marking Using Portable Hand-Operated Instruments",

ASTM E 1710, "Standard Test Method for Measurement of Retroreflective Pavement Marking Materials with CEN-prescribed Geometry Using a Portable Retroreflectometer",

ASTM E 2177, "Standard Test Method for Measuring the Coefficient of Retroreflected Luminance of Pavement Markings in a Standard Condition of Wetness",

ASTM Designation E 2176, "Standard Test Method for Measuring the Coefficient of Retroreflected Luminance of Pavement Markings in a Standard Condition of Continuous Wetting", and

European Standard EN 1436:1997/prA1:2002 "Road marking materials - Road Marking Performance for Road Users"

\section{B.1 Terminology}

1. Luminance coefficient under diffuse illumination, $Q_{d}$ - the ratio of the luminance, $L$, of field of the road marking in the given direction by the illuminance, $E$, on the field, expressed in millicandelas per square meter per lux $\left(\mathrm{mcd} \cdot \mathrm{m}^{-2} \cdot 1 \mathrm{x}^{-1}\right)$.

2. Coefficient of retroreflected luminance, $R_{L}$ - the ratio of the luminance, $L$, of the field of the road marking in the direction of observation by the normal illuminance, $E \perp$, the field perpendicular to the direction of the incident light, expressed in millicandelas per square meter per lux $\left(\mathrm{mcd} \cdot \mathrm{m}^{-2} \cdot 1 \mathrm{x}^{-1}\right)$.

3. Checkpoint area - the evaluation area within each zone of measurement.

4. Portable Retroreflectometer - an instrument that can be used in the field or laboratory for measuring the coefficient of reflected luminance, $R_{L}$, and complies with Test Method ASTM E 1710.

5. Condition of wetness - the test condition created by liberally wetting the road marking and waiting a certain time period after wetting for the water to run off. 
6. Coefficient of retroreflected luminance on a wet surface, $R_{L-W e t}$ - retroreflectivity of horizontal pavement markings in a standard condition of wetness. This condition typically exists after a rainfall has ended and the pavement markings are still wet or as the markings are still wet from dew or humidity.

7. Coefficient of retroreflected luminance on a continuously wetted surface, $R_{L-R a i n}-$ retroreflectivity of horizontal pavement markings in a standard condition of continual wetting. This condition typically exists during a rainfall.

8. Zone of measurement - the road length to measure containing the marking units that appear similar in a visual inspection.

\section{B.2 Measurement Frequency}

The majority of Run-Off-Road sensor validation tests occur in a limited area of a test track or roadway. In such limited areas, the test area, the zone of measurement and the checkpoint area are equivalent.

Validate longer zones of measurement (>300 m) via two checkpoint areas $100 \mathrm{~m}$ long each and separated by at least $100 \mathrm{~m}$ for each kilometer of the test zone. The initial and final $100 \mathrm{~m}$ of the test zone must be included as check point areas. Carry out the necessary remediation and re-verification procedures if a test zone fails to meet the required specifications.

Within each checkpoint area, measure reading sites approximately every $5 \mathrm{~m}$.

Measurements along Broken Longitudinal Horizontal Pavement Markings shall be the same as for continuous markings except there shall be two reading sites per mark and no readings between marks.

\section{B.3 Measurements}

All measurements obtained in the sampling areas are in the direction of traffic flow, i.e., in the direction of the vehicle motion during the sensor test. Measure both single and double lines to determine compliance with prescribed dimensions and the acceptance criteria applied individually to both.

The measurement geometry used to determine the average coefficient of reflective luminance and the luminance coefficient under diffuse illumination shall be $88.76^{\circ}$ for the entrance angle $\left(\beta_{1}\right),\left(\beta_{2}=0^{\circ}\right)$ and $1.05^{\circ}$ for the observation angle. The aperture angles for both the source (if appropriate) and receiver shall not exceed $0.33^{\circ}$. These measurement methods specify the use of portable or mobile reflectometers that can measure pavement markings in accordance with ASTM Test Method E 1710 or CEN 1436. The standard measurement condition (see Figure 33) represents the angles corresponding to a distance of $30 \mathrm{~m}$ for the driver of a passenger car with an eye height of $1.2 \mathrm{~m}$ and a headlight height (if any) of $0.65 \mathrm{~m}$ above the road, commonly called the "CEN 30 meter geometry". 


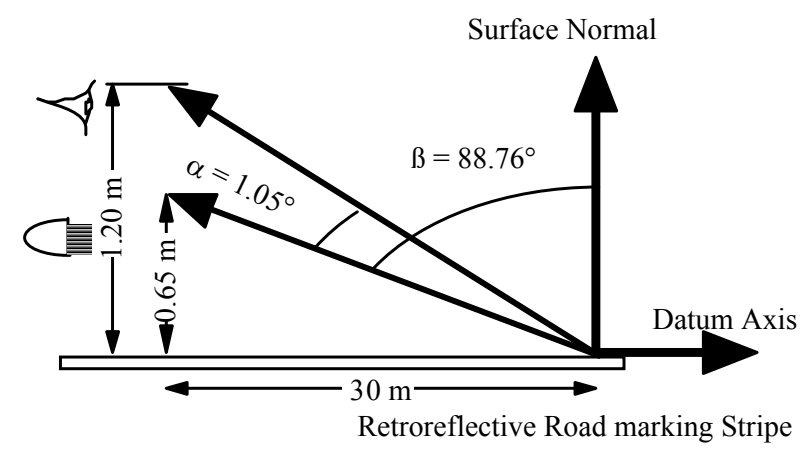

Figure 33. CEN 30 Meter Geometry

Measurements shall be in accordance with the retroreflectometer manufacturer's specification for operation and calibration. When possible a two-point calibration is preferred. The operator shall position the retroreflectometer squarely on or in front of the pavement marking material with the illumination in the direction of the sensor test such that the illuminated measurement area fits within the width of the road marking.

Take measurements within 30 days of the Run-Off-Road sensor test when the road is clean. If using salt, dirt, or a wetting agent to adjust the luminance of the markings, take measurements within 24 hours providing there is no precipitation, high winds, or road cleaning in the test zone between the luminance measurements and the Run-Off-Road sensor test.

\section{B.4 Performance Requirement}

The pavement marking material shall be retroreflecting white or yellow, and shall be readily visible as white or yellow when viewed from the sensor position with automobile headlights at night. Measure the pavement marking after removal of all excess glass spheres. Optionally, use light debris, such as dirt or salt, to retard the marking's reflectivity to achieve a value within the desired range.

The use of the sampling procedure and tables for inspection by attributes (see US Mil-Std 105D "Sampling Procedure and Tables for Inspection by Attributes") forms the basis of the acceptance criteria. Measurements shall proceed until the decision point for rejection is reached, in which case the probability is $95 \%$ that more then $20 \%$ of the pavement markings will fall below or more then $20 \%$ of the pavement markings will fall above the requirement. If less the acceptance number of readings fall below or above the requirements, the probability is $95 \%$ that less then $5 \%$ of the pavement marking will fall below or more then $20 \%$ of the pavement markings will fall above the values specified and the pavement marking is accepted.

\section{B.4.1 Dry Day Road Tests}


The method measures the wet retroreflectivity of horizontal pavement markings.

Measurements shall be taken after a sustained period without precipitation (rain, drizzle, or fog) and the roadway and markings appear dry to the touch, and there is no evidence of residual wetting.

Measure the luminance coefficient under diffuse illumination, $\mathrm{Q}_{\mathrm{d}}$, in accordance with European Standard EN1436: 1997 Annex A. For any set of contiguous 20 readings (approximately $100 \mathrm{~m}$ ), not more then 3 readings may be greater then $130 \mathrm{mcd} \mathrm{m}^{-2} / \mathrm{lx}$, and not more then 3 readings may be less then $100 \mathrm{mcd} \mathrm{m}^{-2} / \mathrm{lx}$ for the markings to be acceptable for Run Off Road Sensor testing.

\section{B.4.2 Dry Night Road Tests}

The method measures the wet retroreflectivity of horizontal pavement markings. Measurements shall be taken after a sustained period without precipitation (rain, drizzle, or fog) and the roadway and markings appear dry to the touch, and there is no evidence of residual wetting.

Measure the coefficient of retroreflected luminance in accordance with ASTM ASTM E 1710. For any set of contiguous 20 readings (approximately $100 \mathrm{~m}$ ), not more then 3 readings may be greater then $130 \mathrm{mcd} \mathrm{m}^{-2} / \mathrm{lx}$, and not more then 3 readings may be less then $100 \mathrm{mcd} \mathrm{m}^{-2} / \mathrm{lx}$ for the markings to be acceptable for Run Off Road Sensor testing.

\section{B.4.3 Wet Road Tests}

This method measures the wet retroreflectivity of horizontal pavement markings in a standard condition of wetness. This condition typically exists after a rainfall has ended and the pavement markings are still wet or as the markings are still wet from dew or humidity. Measurements for tests involving wet roads are similar to methods prescribed in ASTM E 2177. "Standard Test Method for Measuring the Coefficient of Retroreflected Luminance of Pavement markings in a Standard Condition of Wetness".

The operator shall wet the target area with a hand sprayer or bucket. The target area includes the marking and the surrounding area. When using a hand sprayer, wet the area (the stripe and the road surface) for $30 \mathrm{~s}$. The marking and road surface are to be completely flooded. When a hand sprayer is not available, the operator shall slowly pour approximately 10 liters of clean water from a bucket over the measurement area (an approximately $20 \pm 2$ in diameter circle). Pour an even crest of water to temporarily flood the test surface area.

Measure the coefficient of retroreflected luminance of the wetted marking $45 \pm 5 \mathrm{~s}$ after completion of the wetting. For any set of 20 readings (approximately $100 \mathrm{~m}$ ), not more then 3 readings may be greater then $50 \mathrm{mcd} \mathrm{m}^{-2} / \mathrm{lx}$, and not more then 3 readings may be less then $25 \mathrm{mcd} \mathrm{m}^{-2} / 1 \mathrm{x}$ for the markings to be acceptable for Run Off Road Sensor testing.

\section{B.4.4 Continuous Wetting Tests}

This method of measuring of the wet retroflective properties of pavement markings utilizes a method of continuously wetting the marking during measurement with the retroreflectometer and closely follows ASTM E 2176, "Standard Test Method for Measuring the Coefficient of Retroreflected luminance of Pavement markings in a 
Standard Condition of Continuous Wetting". Use the pavement marking's measured performance in conditions of continuous wetting to characterize the performance of the marking on the road as water is continuously falling on it.

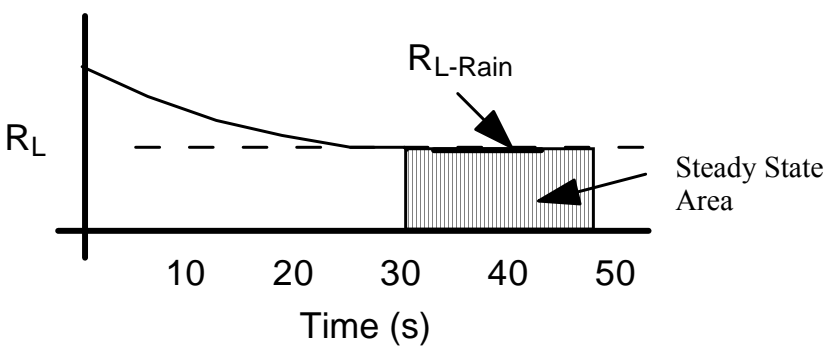

Figure 34 Definition of $\mathbf{R}_{\mathrm{L}-\text { Rain }}$

The operator shall position and adjust the water spray with the nozzle such that it provides an even spray covering the whole area to be. The spray shall cover an area that is $25 \%$ greater then the measuring field measured (generally a $20 \pm 2$ in diameter circle). The variation between the greatest and lowest intensity within the area shall not be greater then the ratio $1.7: 1$. The average spray shall be approximately $20 \mathrm{~mm} / \mathrm{h}$ (heavy rain).

The operator shall take retroreflectometer readings every $10 \mathrm{~s}$ until steady state occurs (commonly between $30 \mathrm{~s}$ and $60 \mathrm{~s}$ ). For any set of 20 readings (approximately $100 \mathrm{~m}$ ), not more then 3 readings may be greater then $50 \mathrm{mcd} \mathrm{m}^{-2} / 1 \mathrm{x}$, and not more then 3 readings may be less then $25 \mathrm{mcd} \mathrm{m}^{-2} / \mathrm{lx}$ for the markings to be acceptable for Run Off Road Sensor testing.

\section{B.5 Reporting}

The test report shall include:

1. the date and time,

2. the location of the test in the form of roadway number, cardinal points, linear distance from the cardinal points, traffic direction, and line identification,

3. the description of the instrumentation and the test method used,

4. the intended road condition (dry, wet, wetting),

5. the readings recorded in millicandelas per square meter per lux $\left(\mathrm{mcd} \bullet \mathrm{m}^{-2} \cdot 1 \mathrm{x}^{-1}\right)$. 